Florida International University FIU Digital Commons

$11-24-1999$

\title{
Numerical calculation of diffraction coefficients from building edges using FDTD method
}

Weiting Cai

Florida International University

DOI: $10.25148 /$ etd.FI14052530

Follow this and additional works at: https://digitalcommons.fiu.edu/etd

Part of the Electrical and Computer Engineering Commons

\section{Recommended Citation}

Cai, Weiting, "Numerical calculation of diffraction coefficients from building edges using FDTD method" (1999). FIU Electronic Theses and Dissertations. 1966.

https://digitalcommons.fiu.edu/etd/1966

This work is brought to you for free and open access by the University Graduate School at FIU Digital Commons. It has been accepted for inclusion in FIU Electronic Theses and Dissertations by an authorized administrator of FIU Digital Commons. For more information, please contact dcc@fiu.edu. 
FLORIDA INTERNATIONAL UNIVERSITY

Miami, Florida

\title{
NUMERICAL CALCULATION OF DIFFRACTION COEFFICIENTS FROM BUILDING EDGES USING FDTD METHOD
}

\author{
A thesis submitted in partial fulfillment of the \\ requirements for the degree of \\ MASTER OF SCIENCE \\ in
}

ELECTRICAL ENGINEERING

by

Weiting Cai

1999 
To: Dean Gordon R. Hopkins

College of Engineering

This thesis, written by Weiting Cai, and entitled Numerical Calculation of Diffraction Coefficients from Building Edges Using FDTD Method, having been approved in respect to style and intellectual content, is referred to you for judgment.

We have read this thesis and recommend that it be approved.

Dr. Subbarao V. Wunnava

Dr. Kazimierz Siwiak

$\widehat{\text { Dr. Tadeusz M X Babij, Major Professor }}$

Date of Defense: November 24th, 1999

The thesis of Weiting Cai is approved.

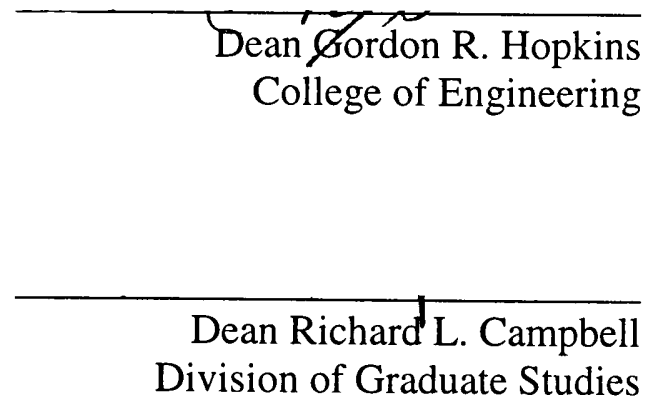

Florida International University, 1999 
(C) Copyright 1999 by Weiting Cai

All rights reserved. 


\section{DEDICATION}

I dedicate this thesis to

my father Hanxiang Cai,

my mother Suyun Zhu,

my sister Weiye Cai,

my teachers,

and my motherland China! 


\section{ACKNOWLEDGMENTS}

First of all, I would like to thank Dr. Tadeusz Babij, my Major Professor who gave me patient advising from the beginning and guided me into interesting subjects. Dr. Babij often directed me in doing exercises, reading references and spent precious time for me through this thesis research. Thanks a lot for his great help and strong support.

I would express my gratitude to Dr. Kazimierz Siwiak from Motorola Company for providing us the research opportunities and resources [under grant number 571843300]. Dr. Siwiak was constantly taking time to comment on my work and gave me insight into helpful information.

I want to thank Dr. Subbarao Wunnava as my thesis committee member. I learned much from his wonderful classes in which everybody was thinking actively.

I am grateful to Dr. Malcolm Heimer and Thomas Gilbar for their tremendous help at the start of my study. I would like to thank Dr. Kang Yen, Dr. Mark Hagmann and Dr. Manuel Cereijo who taught me interesting classes and encouraged me continuously.

I would thank Pat, Marbeth and Nancy, for their kindness and concern during the course of my education in FIU. Thanks to members in High-speed Electronics Lab and my classmates whom I feel happy to be with in the past time. 
ABSTRACT OF THE THESIS

\title{
NUMERICAL CALCULATION OF DIFFRACTION COEFFICIENTS \\ FROM BUILDING EDGES USING FDTD METHOD
}

\author{
by \\ Weiting Cai \\ Florida International University, 1999 \\ Miami, Florida \\ Professor Tadeusz M. Babij, Major Professor
}

Finite Difference Time Domain (FDTD) Method and software are applied to obtain diffraction waves from modulated Gaussian plane wave illumination for right angle wedges and Fast Fourier Transform (FFT) is used to get diffraction coefficients in a wideband in the illuminated lit region. Theta and Phi polarization in 3-dimensional, TM and TE polarization in 2-dimensional cases are considered respectively for soft and hard diffraction coefficients. Results using FDTD method of perfect electric conductor (PEC) wedge are compared with asymptotic expressions from Uniform Theory of Diffraction (UTD). Extend the PEC wedges to some homogenous conducting and dielectric building materials for diffraction coefficients that are not available analytically in practical conditions. 


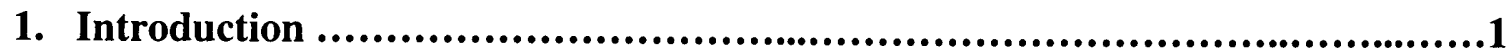

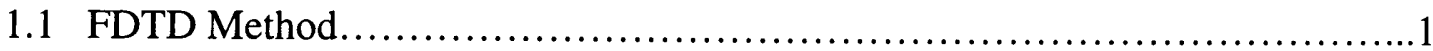

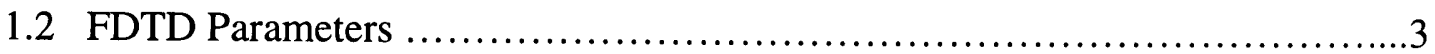

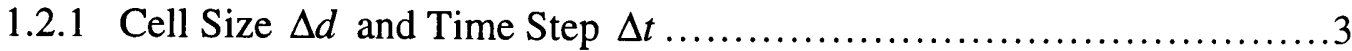

1.2.2 Plane Wave Source...............................................5

1.2.2.1 Gaussian Envelop ...........................................5

1.2.2.2 Modulated Gaussian Pulse.....................................6

1.2.3 Boundary Condition.................................................. 9

1.3 Scattering Application Using FDTD .................................... 10

1.3.1 Near Zone - Far Zone Transformation.............................. 10

1.3.2 Radar Cross Section (RCS) ..........................................11

1.3.3 RCS of Perfect Conducting Plate....................................12

2. Validation of Diffraction Coefficients from Electromagnetic Software............16

2.1 Diffraction Field Calculation ............................................16

2.2 Diffraction Coefficients Validation of PEC Wedge .............................. 17

2.3 Diffraction Coefficients of Building Wedges................................. 17

2.4 Experimental Validation................................................... 18

3. 3-Dimensional Diffraction Coefficient................................................19

3.1 Diffraction Phenomenon................................................... 19

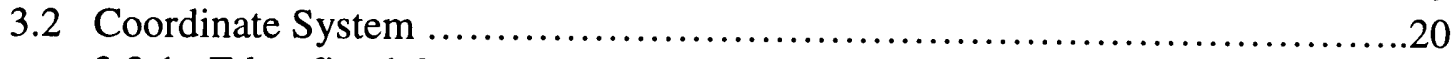

3.2.1 Edge-fixed Coordinate System....................................20

3.2.2 Ray-fixed Coordinate System.......................................22

3.3 3-Dimensional Diffraction Coefficient of PEC Wedge...........................23

3.3.1 Asymptotic 3-Dimensional Diffraction Coefficient

of PEC Wedge....................................................24

3.3.2 3-Dimensional Diffraction Coefficient of PEC Wedge

Using FDTD Method.

3.3.2.1 Diffraction Coefficients of PEC Wedge for $\phi^{\prime}=150^{\circ}, \beta^{\prime}=70^{\circ}, \phi=35^{\circ}$.

3.3.2.2 Diffraction Coefficients of PEC Wedge for $\phi^{\prime}=150^{\circ}, \beta^{\prime}=70^{\circ}, \phi=40^{\circ}$.

3.3.2.3 Diffraction Coefficients of PEC Wedge for $\phi^{\prime}=150^{\circ}, \beta^{\prime}=70^{\circ}, \phi=45^{\circ}$.

3.3.2.4 Diffraction Coefficients of PEC Wedge for $\phi^{\prime}=150^{\circ}, \beta^{\prime}=70^{\circ}, \phi=50^{\circ}$.

3.3.2.5 Diffraction Coefficients of PEC Wedge for $\phi^{\prime}=150^{\circ}, \beta^{\prime}=70^{\circ}, \phi=60^{\circ}$ 
3.3.2.6 Diffraction Coefficients of PEC Wedge for $\phi^{\prime}=150^{\circ}, \beta^{\prime}=70^{\circ}, \phi=70^{\circ}$.

3.3.2.7 Diffraction Coefficients of PEC Wedge

for $\phi^{\prime}=150^{\circ}, \beta^{\prime}=70^{\circ}, \phi=80^{\circ}$

3.3.2.8 Diffraction Coefficients of PEC Wedge

for $\phi^{\prime}=150^{\circ}, \beta^{\prime}=70^{\circ}, \phi=100^{\circ}$.

3.4 3-Dimensional Diffraction Coefficient of Dielectric Wedges

3.4.1 Diffraction Coefficients of Dielectric Wedge

for $\phi^{\prime}=150^{\circ}, \beta^{\prime}=70^{\circ}, \phi=35^{\circ}$.

3.4.1.1 Diffraction Coefficients for Wedge

$\left(\varepsilon_{r}, \sigma=100,100\right)$ in $\phi=35^{\circ}$.

3.4.1.2 Diffraction Coefficients for Wedge

$\left(\varepsilon_{r}, \sigma=12,0.1\right)$ in $\phi=35^{\circ}$.

3.4.1.3 Diffraction Coefficients for Wedge

$\left(\varepsilon_{r}, \sigma=3,0.01\right)$ in $\phi=35^{\circ}$

3.4.2 Diffraction Coefficients of Dielectric Wedge

for $\phi^{\prime}=150^{\circ}, \beta^{\prime}=70^{\circ}, \phi=40^{\circ}$.

3.4.3 Diffraction Coefficients of Dielectric Wedge

for $\phi^{\prime}=150^{\circ}, \beta^{\prime}=70^{\circ}, \phi=45^{\circ}$

3.4.4 Diffraction Coefficients of Dielectric Wedge

for $\phi^{\prime}=150^{\circ}, \beta^{\prime}=70^{\circ}, \phi=50^{\circ}$.

3.4.5 Diffraction Coefficients of Dielectric Wedge

for $\phi^{\prime}=150^{\circ}, \beta^{\prime}=70^{\circ}, \phi=60^{\circ}$

3.4.6 Diffraction Coefficients of Dielectric Wedge

for $\phi^{\prime}=150^{\circ}, \beta^{\prime}=70^{\circ}, \phi=70^{\circ}$.

3.4.7 Diffraction Coefficients of Dielectric Wedge

for $\phi^{\prime}=150^{\circ}, \beta^{\prime}=70^{\circ}, \phi=80^{\circ}$

3.4.8 Diffraction Coefficients of Dielectric Wedge

for $\phi^{\prime}=150^{\circ}, \beta^{\prime}=70^{\circ}, \phi=100^{\circ}$

4. 2-Dimensional Diffraction Coefficient.

4.1 2-Dimensional Diffraction............................................. 70

4.2 2-Dimensional Diffraction Coefficient of PEC Wedge...........................71

4.2.1 Asymptotic 2-Dimensional Diffraction Coefficient of PEC Wedge.......71

4.2.2 2-Dimensional Diffraction Coefficient of PEC Wedge

Using FDTD method.

4.2.2.1 Total Fields and Scattered Fields.

4.2.2.2 2-Dimensional Diffraction Coefficient of PEC Wedge

in Shadow Region III around Incident Shadow Boundary

4.2.2.3 2-Dimensional Diffraction Coefficient of PEC Wedge in Region II around Incident Shadow Boundary. 
5. Conclusions and Future Work.....................................................85

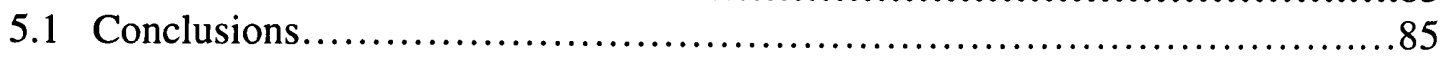

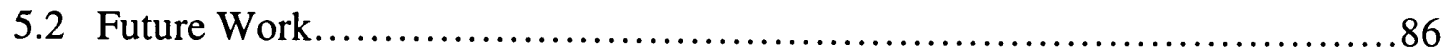

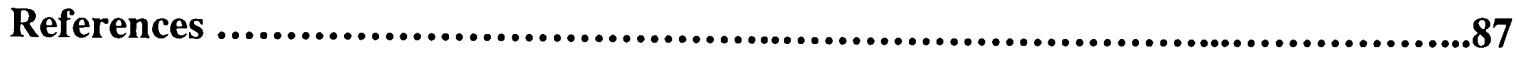




\section{LIST OF FIGURES}

FIGURE

PAGE

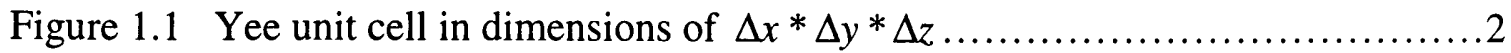

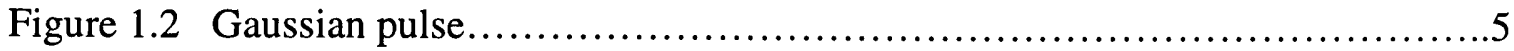

Figure 1.3 Find the frequency $f_{c}$ for the maximum amplitude in spectrum $\left(f_{0}=850 \mathrm{MHz}, \beta=32\right.$ time steps, $\left.\Delta t=27.188 \mathrm{ps}\right) \ldots \ldots \ldots \ldots \ldots \ldots \ldots \ldots \ldots$

Figure 1.4 Waveform of modulated Gaussian pulse

( $f_{0}=850 \mathrm{MHz}, \beta=32$ time steps, $\Delta t=27.188 \mathrm{ps}, \mathrm{A}=1$ )

Figure 1.5 Analog Fourier transform $|X(f)|$ of modulated Gaussian pulse $\left(f_{0}=850 \mathrm{MHz}, \beta=32\right.$ time steps, $\left.\Delta t=27.188 \mathrm{ps}, \mathrm{A}=1\right) \ldots \ldots \ldots \ldots \ldots \ldots \ldots . .8$

Figure 1.6 DFT $|\hat{X}(f)|$ of modulated Gaussian pulse ( $f_{0}=850 \mathrm{MHz}, \beta=32$ time steps, $\Delta t=27.188 \mathrm{ps}, \mathrm{A}=1$ ).

Figure 1.7 Near zone to far zone transformation................................. 10

Figure 1.8 Geometry of the perfect conducting plate scatterer inside the problem space.

Figure 1.9 Far zone backscatter field from perfect conducting plate with normally incident (from $+z$ direction) Gaussian pulse plane wave by FDTD.

Figure 1.10 Far zone backscatter radar cross section from perfect conducting plate with normally incident Gaussian pulse plane wave.

Figure 1.11 Far zone co-polarized backscatter field for perfect conducting plate with $\phi$-polarized Gaussian pulse plane wave incident from $\phi=30^{\circ}, \theta=45^{\circ}$ by FDTD.

Figure 1.12 Far zone co-polarized backscatter radar cross section from perfect conducting plate.

Figure 1.13 Far zone cross-polarized backscatter field for perfect conducting plate with $\phi$-polarized Gaussian pulse plane wave incident from $\phi=30^{\circ}, \theta=45^{\circ}$ by FDTD 
Figure 1.14 Far zone cross-polarized backscatter radar cross section from perfect conducting plate.

Figure 3.1 Diffraction around buildings

Figure 3.2 Shadow regions and boundaries caused by diffraction .20

Figure 3.3 Edge-fixed coordinate system...................................... 21

Figure 3.4 3-Dimensional diffraction cone......................................21

Figure 3.5 Ray-fixed coordinate system.........................................22

Figure 3.6 Geometry of the structure inside problem space to get diffraction coefficients from $\phi^{\prime}=150^{\circ}, \beta^{\prime}=70^{\circ}$ plane wave incidence.

Figure 3.7 Scattered Fields Consideration from side view and top view .30

Figure 3.8 Geometry of structure and problem space to get the diffraction coefficient for $\phi^{\prime}=150^{\circ}, \beta^{\prime}=70^{\circ}$ and $\phi=35^{\circ}$ in $\mathrm{R}=1.06 \mathrm{~m}$.

Figure $3.9 \theta$-polarized incident modulated Gaussian plane wave inside the problem space from $\phi_{\text {ftrd }}^{i}=210^{\circ} \theta_{\text {ftrd }}^{i}=110^{\circ}\left(f_{0}=850 \mathrm{MHz}\right.$, pulse width $=32$ time

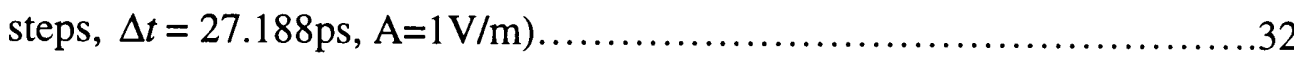

Figure 3.10 Spectrum of the $\theta$-polarized incident modulated Gaussian plane wave....33

Figure 3.11 Scattered field Ex at receiver from $\theta$-polarized plane wave incident on PEC wedge for $\phi=35^{\circ}$

Figure 3.12 Scattered field Ey at receiver from $\theta$-polarized plane wave incident on PEC wedge for $\phi=35^{\circ}$

Figure 3.13 Scattered field Ez at receiver from $\theta$-polarized plane wave incident on PEC wedge for $\phi=35^{\circ}$.

Figure 3.14 Scattered field at receiver from $\theta$-polarized plane wave incident on PEC wedge for $\phi=35^{\circ}$ .35

Figure 3.15 Extract the diffraction field from $\theta$-polarized plane wave incident on PEC wedge for $\phi=35^{\circ}$ using time-gating. 
Figure 3.16 Spectrum of the diffraction field $\theta$-polarized plane wave incident on PEC wedge for $\phi=35^{\circ}$

Figure 3.17 Comparison of soft diffraction coefficient $D_{s}$ from PEC wedge for $\phi=35^{\circ}$ by asymptotic and FDTD.

Figure 3.18 Scattered field Ex at receiver from $\phi$-polarized plane wave incident on PEC wedge for $\phi=35^{\circ}$

Figure 3.19 Scattered field Ey at receiver from $\phi$-polarized plane wave incident on PEC wedge for $\phi=35^{\circ}$

Figure 3.20 Scattered field at receiver from $\phi$-polarized plane wave incident on PEC wedge for $\phi=35^{\circ}$

Figure 3.21 Extract the diffraction field from $\phi$-polarized plane wave incident on PEC wedge for $\phi=35^{\circ}$ using time-gating.

Figure 3.22 Spectrum of the diffraction field $\phi$-polarized plane wave incident on PEC wedge for $\phi=35^{\circ}$

Figure 3.23 Comparison of hard diffraction coefficient $D_{h}$ from PEC wedge for $\phi=35^{\circ}$ by asymptotic and FDTD 39

Figure 3.24 Geometry of structure and problem space to get the diffraction coefficient for $\phi^{\prime}=150^{\circ}, \beta^{\prime}=70^{\circ}$ and $\phi=40^{\circ}$ in $\mathrm{R}=1.06 \mathrm{~m}$

Figure 3.25 Comparison of soft and hard diffraction coefficients for PEC wedge at $1.06 \mathrm{~m}\left(\beta^{\prime}=70^{\circ}, \phi^{\prime}=150^{\circ}, \phi=40^{\circ}\right)$ by asymptotic and FDTD

Figure 3.26 Geometry of structure and problem space to get the diffraction coefficient for $\phi^{\prime}=150^{\circ}, \beta^{\prime}=70^{\circ}$ and $\phi=45^{\circ}$ in $\mathrm{R}=1.06 \mathrm{~m}$

Figure 3.27 Comparison of soft and hard diffraction coefficients for PEC wedge at $1.06 \mathrm{~m}\left(\beta^{\prime}=70^{\circ}, \phi^{\prime}=150^{\circ}, \phi=45^{\circ}\right)$ by asymptotic and FDTD

Figure 3.28 Geometry of structure and problem space to get the diffraction coefficient for $\phi^{\prime}=150^{\circ}, \beta^{\prime}=70^{\circ}$ and $\phi=50^{\circ}$ in $\mathrm{R}=1.06 \mathrm{~m}$ 
Figure 3.29 Comparison of soft and hard diffraction coefficients for PEC wedge at $1.06 \mathrm{~m}\left(\beta^{\prime}=70^{\circ}, \phi^{\prime}=150^{\circ}, \phi=50^{\circ}\right)$ by asymptotic and FDTD

Figure 3.30 Geometry of structure and problem space to get the diffraction coefficient for $\phi^{\prime}=150^{\circ}, \beta^{\prime}=70^{\circ}$ and $\phi=60^{\circ}$ in $\mathrm{R}=1.06 \mathrm{~m}$.

Figure 3.31 Comparison of soft and hard diffraction coefficients for PEC wedge at $1.06 \mathrm{~m}\left(\beta^{\prime}=70^{\circ}, \phi^{\prime}=150^{\circ}, \phi=60^{\circ}\right)$ by asymptotic and FDTD

Figure 3.32 Geometry of structure and problem space to get the diffraction coefficient for $\phi^{\prime}=150^{\circ}, \beta^{\prime}=70^{\circ}$ and $\phi=70^{\circ} \mathrm{R}=$ in $1.06 \mathrm{~m}$.

Figure 3.33 Comparison of soft and hard diffraction coefficients for PEC wedge at $1.06 \mathrm{~m}\left(\beta^{\prime}=70^{\circ}, \phi^{\prime}=150^{\circ}, \phi=70^{\circ}\right)$ by asymptotic and FDTD

Figure 3.34 Geometry of structure and problem space to get the diffraction coefficient for $\phi^{\prime}=150^{\circ}, \beta^{\prime}=70^{\circ}$ and $\phi=80^{\circ}$ in $\mathrm{R}=1.06$.

Figure 3.35 Comparison of soft and hard diffraction coefficients for PEC wedge at $1.06 \mathrm{~m}\left(\beta^{\prime}=70^{\circ}, \phi^{\prime}=150^{\circ}, \phi=80^{\circ}\right)$ by asymptotic and FDTD.

Figure 3.36 Geometry of structure and problem space to get the diffraction coefficient for $\phi^{\prime}=150^{\circ}, \beta^{\prime}=70^{\circ}$ and $\phi=100^{\circ}$ in $\mathrm{R}=1.06 \mathrm{~m}$

Figure 3.37 Comparison of soft and hard diffraction coefficients for PEC wedge at $1.06 \mathrm{~m}\left(\beta^{\prime}=70^{\circ}, \phi^{\prime}=150^{\circ}, \phi=100^{\circ}\right)$ by asymptotic and FDTD.

Figure 3.38 Comparison of soft and hard diffraction coefficients at $850 \mathrm{MHz}$ to different directions for PEC wedge at $1.06 \mathrm{~m}\left(\beta^{\prime}=70^{\circ}, \phi^{\prime}=150^{\circ}\right)$ by asymptotic and FDTD ................................................48

Figure 3.39 Comparison of soft and hard diffraction coefficients at $1.7 \mathrm{GHz}$ to different directions for PEC wedge at $1.06 \mathrm{~m}\left(\beta^{\prime}=70^{\circ}, \phi^{\prime}=150^{\circ}\right)$ by asymptotic and FDTD.

Figure 3.40 Diffraction field from $\theta$-polarized plane wave incident on wedge $\left(\varepsilon_{r}, \sigma=100,100\right)$ for $\phi=35^{\circ}$ using time-gating.

Figure 3.41 Spectrum of the diffraction field from $\theta$-polarized plane wave incident on wedge $\left(\varepsilon_{r}, \sigma=100,100\right)$ for $\phi=35^{\circ}$ 
Figure 3.42 Soft diffraction coefficient of wedge $\left(\varepsilon_{r}, \sigma=100,100\right)$ for $\phi=35^{\circ}$ using FDTD

Figure 3.43 Diffraction field from $\phi$-polarized plane wave incident on wedge ( $\left.\varepsilon_{r}, \sigma=100,100\right)$ for $\phi=35^{\circ}$ using time-gating.

Figure 3.44 Spectrum of the diffraction field from $\phi$-polarized plane wave incident on wedge $\left(\varepsilon_{r}, \sigma=100,100\right)$ for $\phi=35^{\circ}$

Figure 3.45 Hard diffraction coefficient of wedge $\left(\varepsilon_{r}, \sigma=100,100\right)$ for $\phi=35^{\circ}$ using FDTD

Figure 3.46 Diffraction field from $\theta$-polarized plane wave incident on wedge ( $\varepsilon_{r}, \sigma=12,0.1$ ) for $\phi=35^{\circ}$ using time-gating.

Figure 3.47 Spectrum of the diffraction field from $\theta$-polarized plane wave incident on wedge $\left(\varepsilon_{r}, \sigma=12,0.1\right)$ for $\phi=35^{\circ}$

Figure 3.48 Soft diffraction coefficient of wedge $\left(\varepsilon_{r}, \sigma=12,0.1\right)$ for $\phi=35^{\circ}$ using FDTD .54

Figure 3.49 Diffraction field from $\phi$-polarized plane wave incident on wedge ( $\left.\varepsilon_{r}, \sigma=12,0.1\right)$ for $\phi=35^{\circ}$ using time-gating. .54

Figure 3.50 Spectrum of the diffraction field from $\phi$-polarized plane wave incident on wedge $\left(\varepsilon_{r}, \sigma=12,0.1\right)$ for $\phi=35^{\circ}$

Figure 3.51 Hard diffraction coefficient of wedge $\left(\varepsilon_{r}, \sigma=12,0.1\right)$ for $\phi=35^{\circ}$ using FDTD. 55

Figure 3.52 Diffraction field from $\theta$-polarized plane wave incident on wedge ( $\varepsilon_{r}, \sigma=3,0.01$ ) for $\phi=35^{\circ}$ using time-gating.

Figure 3.53 Spectrum of the diffraction field from $\theta$-polarized plane wave incident on wedge $\left(\varepsilon_{r}, \sigma=3,0.01\right)$ for $\phi=35^{\circ}$.

Figure 3.54 Soft diffraction coefficient of wedge $\left(\varepsilon_{r}, \sigma=3,0.01\right)$ for $\phi=35^{\circ}$ using FDTD. 
Figure 3.55 Diffraction field from $\phi$-polarized plane wave incident on wedge $\left(\varepsilon_{r}, \sigma=3,0.01\right)$ for $\phi=35^{\circ}$ using time-gating

Figure 3.56 Spectrum of the diffraction field from $\phi$-polarized plane wave incident on wedge $\left(\varepsilon_{r}, \sigma=3,0.01\right)$ for $\phi=35^{\circ} \ldots$

Figure 3.57 Hard diffraction coefficient of wedge $\left(\varepsilon_{r}, \sigma=3,0.01\right)$ for $\phi=35^{\circ}$ using FDTD

Figure 3.58 3-Dimensional soft diffraction coefficient for four wedge types for $\phi=35^{\circ}$.

Figure 3.59 3-Dimensional hard diffraction coefficient for four wedge types for $\phi=35^{\circ}$

Figure 3.60 3-Dimensional soft diffraction coefficient for four wedge types for $\phi=40^{\circ}$

Figure 3.61 3-Dimensional hard diffraction coefficient for four wedge types for $\phi=40^{\circ}$

Figure 3.62 3-Dimensional soft diffraction coefficient for four wedge types for $\phi=45^{\circ}$

Figure 3.63 3-Dimensional hard diffraction coefficient for four wedge types for $\phi=45^{\circ}$.

Figure 3.64 3-Dimensional soft diffraction coefficient for four wedge types for $\phi=50^{\circ}$

Figure 3.65 3-Dimensional hard diffraction coefficient for four wedge types for $\phi=50^{\circ}$

Figure 3.66 3-Dimensional soft diffraction coefficient for four wedge types for $\phi=60^{\circ}$

Figure 3.67 3-Dimensional hard diffraction coefficient for four wedge types for $\phi=60^{\circ}$

Figure 3.68 3-Dimensional soft diffraction coefficient for four wedge types for $\phi=70^{\circ}$ 
Figure 3.69 3-Dimensional hard diffraction coefficient for four wedge types for $\phi=70^{\circ}$

Figure 3.70 3-Dimensional soft diffraction coefficient for four wedge types for $\phi=80^{\circ}$

Figure 3.71 3-Dimensional soft diffraction coefficient for four wedge types for $\phi=80^{\circ}$.

Figure 3.72 3-Dimensional soft diffraction coefficient for four wedge types for $\phi=100^{\circ}$.

Figure 3.73 3-Dimensional soft diffraction coefficient for four wedge types for $\phi=100^{\circ}$.

Figure 3.74 Comparison of soft diffraction coefficients at $850 \mathrm{MHz}$ to different directions for four wedge types at $1.06 \mathrm{~m}\left(\beta^{\prime}=70^{\circ}, \phi^{\prime}=150^{\circ}\right)$ by asymptotic and FDTD.

Figure 3.75 Comparison of soft diffraction coefficients at $1.7 \mathrm{GHz}$ to different directions for four wedge types at $1.06 \mathrm{~m}\left(\beta^{\prime}=70^{\circ}, \phi^{\prime}=150^{\circ}\right)$ by asymptotic and FDTD. 68

Figure 3.76 Comparison of hard diffraction coefficients at $850 \mathrm{MHz}$ to different directions for four wedge types at $1.06 \mathrm{~m}\left(\beta^{\prime}=70^{\circ}, \phi^{\prime}=150^{\circ}\right)$ by asymptotic and FDTD

Figure 3.77 Comparison of hard diffraction coefficients at $1.7 \mathrm{GHz}$ to different directions for four wedge types at $1.06 \mathrm{~m}\left(\beta^{\prime}=70^{\circ}, \phi^{\prime}=150^{\circ}\right)$ by asymptotic and FDTD.

Figure 4.1 2-Dimensional diffraction disk .70

Figure 4.2 TM and TE polarization in 2-dimensional diffraction 70

Figure 4.3 Asymptotic soft and hard diffraction coefficients at $850 \mathrm{MHz}$ all around the wedge in $0.8 \mathrm{~m}$ from $80^{\circ}$ incidence

Figure 4.4 Geometry of structure and problem space to get 2-dimensional diffraction coefficient for $\phi^{\prime}=80^{\circ}$ and $\phi=263^{\circ}$ in $\mathrm{R}=0.8 \mathrm{~m}$

Figure 4.5 Diffraction field in TM polarization for $\phi^{\prime}=80^{\circ}, \phi=263^{\circ}$ in $\mathrm{R}=0.8 \mathrm{~m} \ldots . .75$ 
Figure 4.6 Spectrum of the diffraction field in TM polarization

for $\phi^{\prime}=80^{\circ}, \phi=263^{\circ}$ in $\mathrm{R}=0.8 \mathrm{~m}$

Figure 4.7 2-Dimensional soft diffraction coefficient of PEC wedge

for $\phi^{\prime}=80^{\circ}, \phi=263^{\circ}$ in $\mathrm{R}=0.8 \mathrm{~m}$

Figure 4.8 Diffraction field in TE polarization for $\phi^{\prime}=80^{\circ}, \phi=263^{\circ}$ in $\mathrm{R}=0.8 \mathrm{~m} \ldots . .77$

Figure 4.9 Spectrum of the diffraction field in TE polarization

for $\phi^{\prime}=80^{\circ}, \phi=263^{\circ}$ in $\mathrm{R}=0.8 \mathrm{~m}$

Figure 4.10 2-Dimensional hard diffraction coefficient of PEC wedge for $\phi^{\prime}=80^{\circ}$,

$\phi=263^{\circ}$ in $\mathrm{R}=0.8 \mathrm{~m}$ .78

Figure 4.11 Geometry of structure and problem space to get 2-dimensional diffraction coefficient for $\phi^{\prime}=80^{\circ}$ and $\phi=257^{\circ}$ in $\mathrm{R}=0.8 \mathrm{~m}$.

Figure 4.12 2-Dimensional soft and hard diffraction coefficients of PEC wedge for $\phi^{\prime}=80^{\circ}, \phi=257^{\circ}$ in $\mathrm{R}=0.8 \mathrm{~m}$.

Figure 4.13 Diffraction field in TM polarization for $\phi^{\prime}=80^{\circ}, \phi=263^{\circ}$ in $\mathrm{R}=0.8 \mathrm{~m}$ for wedge $\left(\varepsilon_{r}, \sigma=12,0.1\right)$

Figure 4.14 Spectrum of the diffraction field from TM polarization for $\phi^{\prime}=80^{\circ}$, $\phi=263^{\circ}$ in $\mathrm{R}=0.8 \mathrm{~m}$ for wedge $\left(\varepsilon_{r}, \sigma=12,0.1\right)$.

Figure 4.15 2-Dimensional soft diffraction coefficient for $\phi^{\prime}=80^{\circ}, \phi=263^{\circ}$ in $\mathrm{R}=0.8 \mathrm{~m}$ for wedge $\left(\varepsilon_{r}, \sigma=12,0.1\right)$

Figure 4.16 Diffraction field in TE polarization for $\phi^{\prime}=80^{\circ}, \phi=263^{\circ}$ in $\mathrm{R}=0.8 \mathrm{~m}$ for wedge $\left(\varepsilon_{r}, \sigma=12,0.1\right)$.

Figure 4.17 Spectrum of the diffraction field from TE polarization for $\phi^{\prime}=80^{\circ}$, $\phi=263^{\circ}$ in $\mathrm{R}=0.8 \mathrm{~m}$ for wedge $\left(\varepsilon_{r}, \sigma=12,0.1\right)$.

Figure 4.18 2-Dimensional hard diffraction coefficient for $\phi^{\prime}=80^{\circ}, \phi=263^{\circ}$ in $\mathrm{R}=0.8 \mathrm{~m}$ for wedge $\left(\varepsilon_{r}, \sigma=12,0.1\right)$.

Figure 4.19 2-Dimensional soft diffraction coefficient for $\phi^{\prime}=80^{\circ}, \phi=263^{\circ}$ in $\mathrm{R}=0.8 \mathrm{~m}$ for wedges of PEC, $\left(\varepsilon_{r}, \sigma=100,100\right)$ and $\left(\varepsilon_{r}, \sigma=12,0.1\right) \ldots . .83$ 
Figure 4.20 2-Dimensional hard diffraction coefficient for $\phi^{\prime}=80^{\circ}, \phi=263^{\circ}$ in $\mathrm{R}=0.8 \mathrm{~m}$ for wedges of PEC, $\left(\varepsilon_{r}, \sigma=100,100\right)$ and $\left(\varepsilon_{r}, \sigma=12,0.1\right) \ldots . .84$ 


\section{Chapter 1}

\section{Introduction}

Finite Difference Time Domain (FDTD) Method is a time domain technique for the solution of applications in electromagnetics. Using a signal pulse as the source, wide frequency information can be extracted from the FDTD calculation after Fourier Transform. This is extremely useful when resonant frequencies are not known exactly or a broadband result is desired.

\subsection{FDTD Method}

Maxwell equations in linear isotropic medium govern the electrical and magnetic fields in the propagation of electromagnetic waves:

$$
\begin{gathered}
\mu \frac{\partial H}{\partial t}=-\nabla \times E \\
\varepsilon \frac{\partial E}{\partial t}=\nabla \times H-\sigma E
\end{gathered}
$$

In FDTD Cartesian coordinate system the curl operation can be expressed by derivatives for $\mathrm{E}$ and $\mathrm{H}$ fields respectively.

$$
\begin{aligned}
& \nabla \times E_{x}=\left(\frac{\partial E_{z}}{\partial y}-\frac{\partial E_{y}}{\partial z}\right) \bar{x} \\
& \nabla \times E_{y}=\left(\frac{\partial E_{x}}{\partial z}-\frac{\partial E_{z}}{\partial x}\right) \bar{y} \\
& \nabla \times E_{z}=\left(\frac{\partial E_{y}}{\partial x}-\frac{\partial E_{x}}{\partial y}\right) \bar{z}
\end{aligned}
$$




$$
\begin{aligned}
& \nabla \times H_{x}=\left(\frac{\partial H_{z}}{\partial y}-\frac{\partial H_{y}}{\partial z}\right) \bar{x} \\
& \nabla \times H_{y}=\left(\frac{\partial H_{x}}{\partial z}-\frac{\partial H_{z}}{\partial x}\right) \bar{y} \\
& \nabla \times H_{z}=\left(\frac{\partial H_{y}}{\partial x}-\frac{\partial H_{x}}{\partial y}\right) \bar{z}
\end{aligned}
$$

Finite difference is applied for space and time derivatives which makes the calculations implemented on a computer. The whole FDTD problem space is quantized by the unit Yee cell in the rectangular volume containing interest physical structures [1].

$$
\begin{aligned}
& x=i \Delta x \\
& y=j \Delta y \\
& z=k \Delta z
\end{aligned}
$$

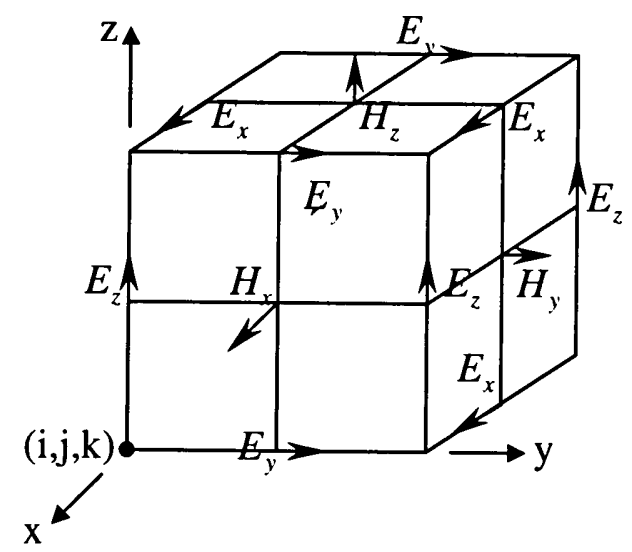

Figure 1.1 Yee unit cell in dimensions of $\Delta x * \Delta y * \Delta z$

The time domain is also discretized by time steps

$$
t=n \Delta t
$$

The electric and magnetic fields on the Yee cells are represented by space and time increments. 


$$
\begin{gathered}
E(x, y, z, t) \rightarrow E^{n}(i, j, k) \\
H(x, y, z, t) \rightarrow H^{n-\frac{1}{2}}(i, j, k)
\end{gathered}
$$

After all discrete operations, central difference approximations for space and time

$$
\begin{aligned}
& \frac{\partial f}{\partial x} \rightarrow \frac{f(x+\Delta x / 2, t)-f(x-\Delta x / 2, t)}{\Delta x} \\
& \frac{\partial f}{\partial t} \rightarrow \frac{f(x, t+\Delta t / 2)-f(x, t-\Delta t / 2)}{\Delta t}
\end{aligned}
$$

are used for $\mathrm{E}$ and $\mathrm{H}$ fields respectively in Maxwell equations.

At the beginning of the calculation all fields and sources within the problem space are zero. The six field components $E_{x, y, z}$ and $H_{x, y, z}$ in every cell are updated time step by time step in terms of the incident field, the initial value from last time step and the adjacent scattered fields around it. Because of the half cell length interleaved by the E and $\mathrm{H}$ fields the equations are processed in leap-frog manner: the $\mathrm{E}$ field is solved at a given instant in time, then the $\mathrm{H}$ field is solved at the next instant in time.

\subsection{FDTD Parameters}

The FDTD calculation parameters are defined by the desired object and the frequencies of interest [2].

\subsubsection{Cell Size $\Delta d$ and Time Step $\Delta t$}

The choice of cell size $\Delta d(\Delta x, \Delta y, \Delta z)$ and time step $\Delta t$ is desired by reasons of accuracy and algorithm stability respectively. 
$\Delta d$ should be small enough to ensure the accuracy of the computed spatial derivatives of the electromagnetic fields and to permit resolution of the principal surface or volumetric details of the structure modeled. It's required that $\Delta d$ be less than $\frac{1}{10}$ of the smallest wavelength corresponding to the highest frequency of interest. For problems containing dielectric materials, the wavelength is shorter and the cell size $\Delta d$ needs to be smaller than that if only free space and perfect conductors were considered.

To maintain stability, waves can not propagate more than one cell per time step. The Courant limit of time step size $\Delta t$ for stability is $[2,3]$

$$
\Delta t \leq \frac{1}{v \sqrt{\frac{1}{(\Delta x)^{2}}+\frac{1}{(\Delta y)^{2}}+\frac{1}{(\Delta z)^{2}}}}
$$

When the equality holds, the discretized wave can approximate the actual wave propagation very closely. In most situations, more accurate results will not be obtained by using smaller time step $\Delta t$ [2]. If $\Delta x=\Delta y=\Delta z=\Delta d$, the maximum time step in free space with velocity of propagation $\mathrm{v}$ as the speed of light $\mathrm{c}$ is

$$
\Delta t=\frac{\Delta d}{c \cdot \sqrt{3}}
$$

The highest valid frequency $f_{\max }$ and the smallest wavelength $\lambda_{\min }$ are

$$
f_{\max }=\frac{c}{\lambda_{\min }}=\frac{c}{10 \cdot \Delta d}=\frac{1}{10 \sqrt{3} \cdot \Delta t}
$$


The total number of time steps $T$ is estimated at lease for fields to get fully interaction with the object after total propagation time $T \Delta t$ is passed.

\subsubsection{Plane Wave Source}

Plane wave source begins outside the problem space and propagates through it. The incident angle $(\phi, \theta)$ according to scattering convention are taken as the angle the incident wave comes from and not the angle of the propagation vector.

\subsubsection{Gaussian Envelop}

Gaussian envelop pulse $f(t)=A e^{-\alpha(t-\beta \Delta t)^{2}}$

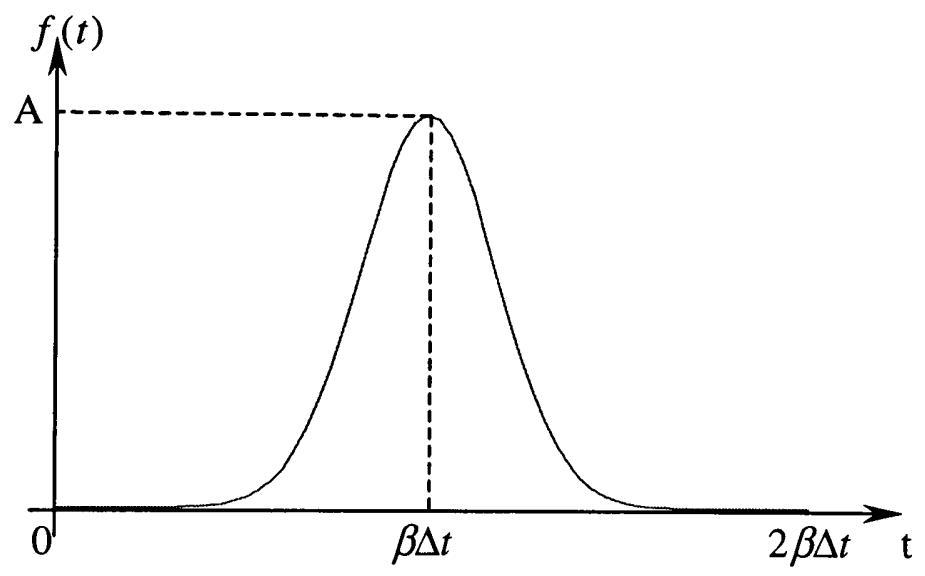

Figure 1.2 Gaussian pulse

The span of the Gaussian pulse is 0 to $2 \beta \Delta t . \alpha$ is the attenuation factor. Pulse width $\beta$ is the number of time steps between the truncation value $\left(e^{-16} A\right.$ in FDTD) and the amplitude $\mathrm{A}$ at the center.

$$
\alpha(\beta \Delta t)^{2}=16
$$




\subsubsection{Modulated Gaussian Pulse}

Modulated Gaussian Pulse is sinusoid at frequency $f_{0}$ with Gaussian Envelope $A e^{-\alpha t^{2}}$ and time delay $\beta \Delta t$

$$
f(t)=A e^{-\alpha(t-\beta \Delta t)^{2}} \sin \left[2 \pi f_{0}(t-\beta \Delta t)\right]
$$

Assume the spectrum of the modulated Gaussian pulse is $X(f)$

$$
\begin{aligned}
|X(f)| & =\left|f t\left[A e^{-\alpha t^{2}} \sin \left(2 \pi f_{0} t\right)\right]\right| \\
& =\left|\int_{-\infty}^{\infty} A e^{-\alpha t^{2}} \cdot \frac{\left(e^{j 2 \pi f_{0} t}-e^{-j 2 \pi f_{0} t}\right)}{2 j} \cdot e^{-j 2 \pi f t} d t\right| \\
& =\frac{A}{2} \sqrt{\frac{\pi}{\alpha}}\left[e^{-\frac{\pi^{2}}{\alpha}\left(f-f_{0}\right)^{2}}-e^{-\frac{\pi^{2}}{\alpha}\left(f+f_{0}\right)^{2}}\right]
\end{aligned}
$$

The frequency $f_{c}$ corresponding to the maximum value in $|X(f)|$ will be higher than the modulated frequency $f_{0}$.

$$
\begin{gathered}
\frac{d[|X(f)|]}{d f}=0 \\
e^{\frac{4 \pi^{2} f_{0}}{\alpha} f_{c}}\left(f_{c}-f_{0}\right)=f_{c}+f_{0} \\
e^{\frac{4 \pi^{2} f_{0}}{\alpha} f_{c}}=1+\frac{2 f_{0}}{f_{c}-f_{0}}
\end{gathered}
$$

If the modulated frequency $f_{0}=850 \mathrm{MHz}, \beta=32$ time steps, $\Delta t=27.188 \mathrm{ps}$, $\alpha=\frac{16}{(\beta \Delta t)^{2}}=2.1138 \times 10^{19}$ 


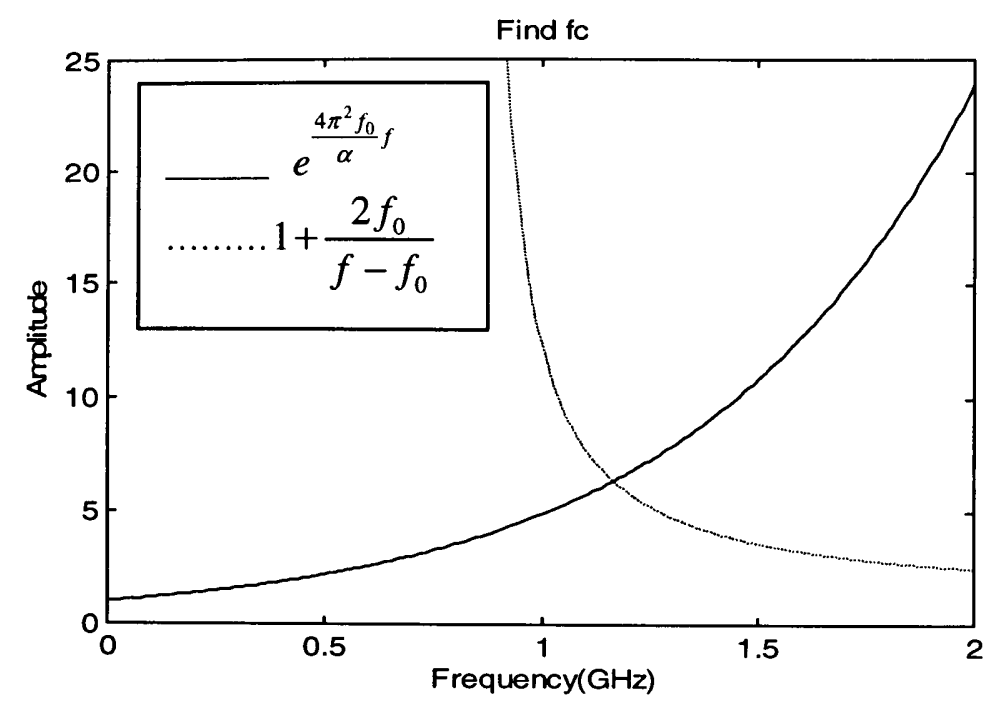

Figure 1.3 Find the frequency $f_{c}$ for the maximum amplitude in spectrum ( $f_{0}=850 \mathrm{MHz}, \beta=32$ time steps, $\Delta t=27.188 \mathrm{ps}$ )

The common value $f_{c}$ in left and right side (1.11) is about $1.2 \mathrm{GHz}$. So the modulated Gaussian pulse at modulated frequency of $850 \mathrm{MHz}$ will have about the same level of energy at the left side $850 \mathrm{MHz}$ and the right side $1.7 \mathrm{GHz}$.

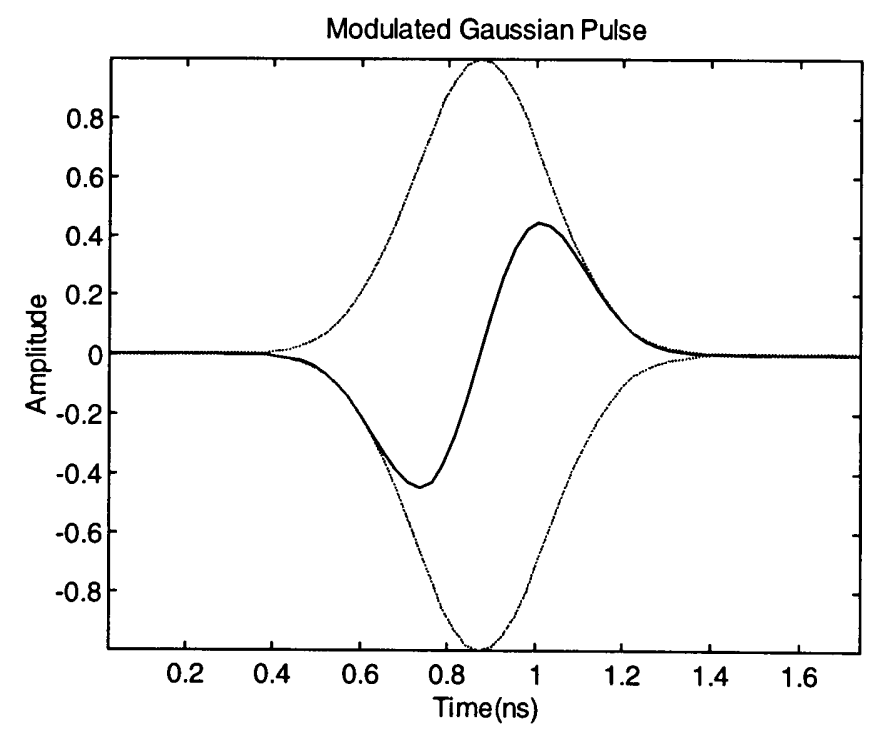

Figure 1.4 Waveform of modulated Gaussian pulse with Gaussian envelop ( $f_{0}=850 \mathrm{MHz}, \beta=32$ time steps, $\Delta t=27.188 \mathrm{ps}, \mathrm{A}=1$ ) 
From (1.10) to get the analog Fourier transform $|X(f)|$

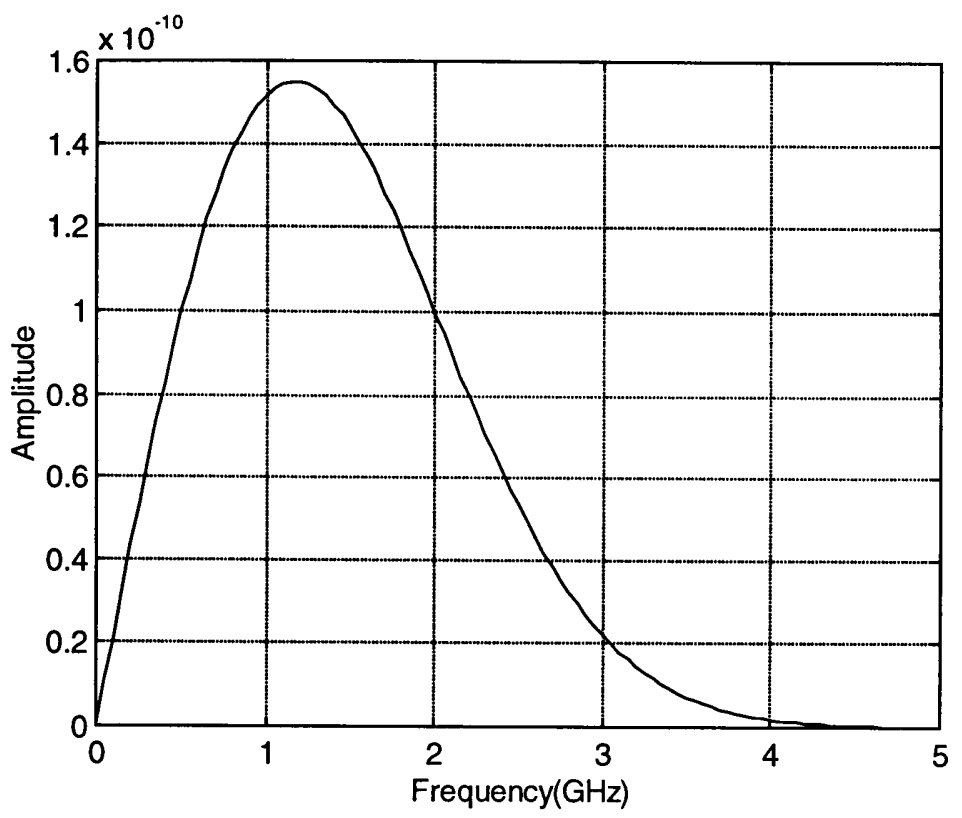

Figure 1.5 Analog Fourier transform $|X(f)|$ of modulated Gaussian pulse ( $f_{0}=850 \mathrm{MHz}, \beta=32$ time steps, $\Delta t=27.188 \mathrm{ps}, \mathrm{A}=1$ )

Because of the differencing characteristic all following spectrums are from Discrete Fourier Transform (DFT) $\hat{X}(f)$ or Fast Fourier Transform (FFT). The difference between analog and discrete Fourier Transform is a constant.

$$
X(f)=\Delta t \cdot \hat{X}(f)
$$

$\Delta t$ is the sample interval as time step in FDTD. 


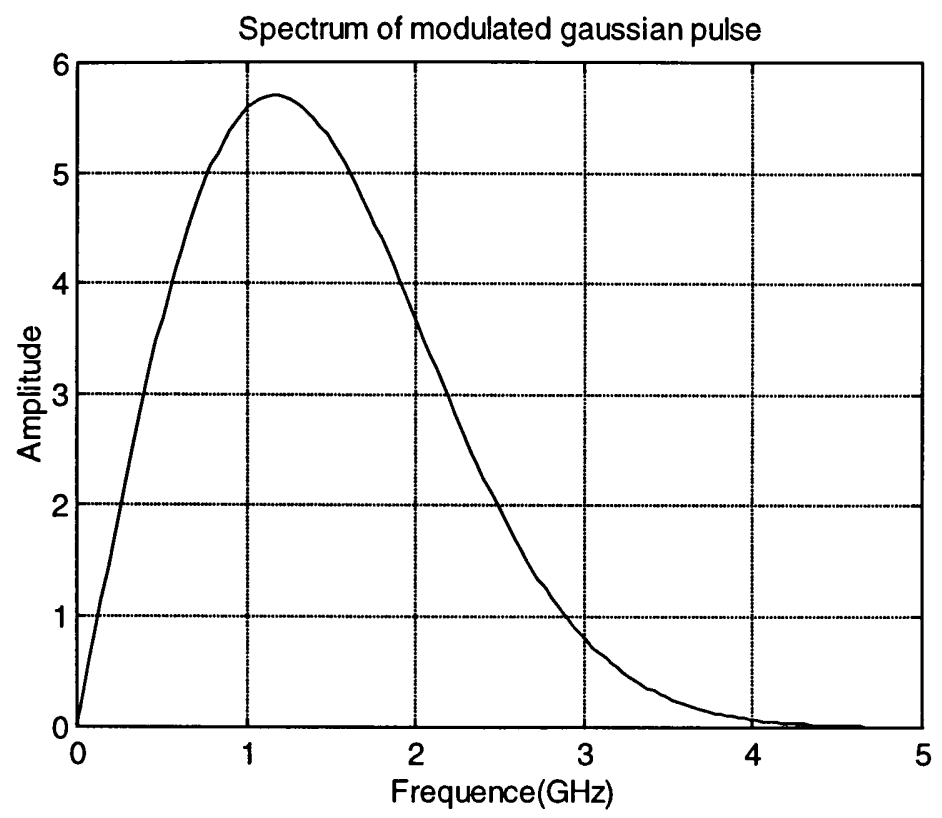

Figure 1.6 DFT $|\hat{X}(f)|$ of modulated Gaussian pulse $\left(f_{0}=850 \mathrm{MHz}, \beta=32\right.$ time steps, $\Delta t=27.188 \mathrm{ps}, \mathrm{A}=1$ )

\subsubsection{Boundary Condition}

The absorbing boundary condition (ABC) simulates the effect of free space beyond the boundary of the problem space with minimum reflection. The default boundary condition in FDTD is a second-order stabilized radiation boundary. It could work well when the scattered fields illuminate the outer boundary normally but not so well as the incident angle moves away from normal and even worse for the scattered fields around the corners of the problem space. The minimum free space between the structure and outer boundary is 10 cells, although 15 of more are sometimes required for accurate results. 


\subsection{Scattering Application Using FDTD}

Far field Radar Cross Section (RCS) response of scatterer is determined by near zone to far zone transformation of equivalent scattering fields obtained in near zone inside FDTD problem space $[4,5]$.

\subsubsection{Near Zone - Far Zone Transformation}

Compute the near zone vector potentials on a closed six-sided rectangular surface which is inside the problem space and fully surrounding the arbitrary-shaped scatterer

$$
\begin{aligned}
& W(t)=\frac{1}{4 \pi R c} \frac{\partial}{\partial t}\left\{\int_{s^{\prime}} J_{s}\left(t+\left(\overline{r^{\prime}} \cdot \bar{r}\right) / c-R / c\right) d s^{\prime}\right\} \\
& U(t)=\frac{1}{4 \pi R c} \frac{\partial}{\partial t}\left\{\int_{s^{\prime}} M_{s}\left(t+\left(\overline{r^{\prime}} \cdot \bar{r}\right) / c-R / c\right) d s^{\prime}\right\}
\end{aligned}
$$
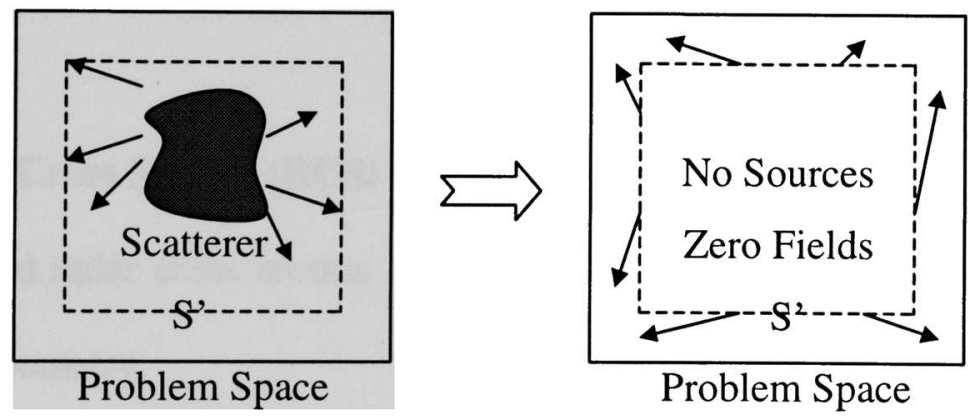

Figure 1.7 Near zone to far zone transformation

$J_{s}(t)=\bar{n} \times H(t), M_{s}(t)=-\bar{n} \times E(t)$ are the time domain electric and magnetic scattered surface currents from all cell face contributions on the closed surface $S^{\prime} \cdot \bar{n}$ is the surface normal unit. $\mathrm{R}$ is the distance to the far zone point. $\bar{r}$ is the vector to the source point of 
integration on $S^{\prime} \cdot \bar{r}$ is the unit vector to the far zone point. The factor $\frac{1}{R}$ is dropped when showing the transient radiation pattern.

Obtain $W_{x, y, z}$ and $U_{x, y, z}$ in Cartesian coordinate and transfer to spherical system $W_{\theta, \phi}$ and $U_{\theta, \phi}$ respectively for far zone angle $(\theta, \phi)$

$$
\begin{gathered}
W_{\theta}=W_{x} \cos \theta \cos \phi+W_{y} \cos \theta \sin \phi-W_{z} \sin \theta \\
W_{\phi}=-W_{x} \sin \phi+W_{y} \cos \phi
\end{gathered}
$$

Far zone electric fields $E_{\theta}$ and $E_{\phi}$ are obtained by

$$
\begin{aligned}
& E_{\theta}=-\eta W_{\theta}-U_{\phi} \\
& E_{\phi}=-\eta W_{\phi}+U_{\theta}
\end{aligned}
$$

$\eta$ is the impedance of free space.

\subsubsection{Radar Cross Section (RCS)}

Far field radar cross section $\sigma$ defines the power gain from the scatterer. In 3dimensional geometry,

$$
\sigma_{3 D}=\lim _{R \rightarrow \infty}\left[4 \pi R^{2} \frac{\left|f f t\left(E^{\text {scattered }}\right)\right|^{2}}{\left|f f t\left(E^{\text {incident }}\right)\right|^{2}}\right]
$$

From (1.12) to (1.17) the distance factor $\frac{1}{R}$ in E field makes $\frac{1}{R^{2}}$ in power and $\mathrm{R}$ will be suppressed in RCS expression of equation (1.18). 


\subsubsection{RCS of Perfect Conducting Plate}

Far zone backscatter fields and wideband RCS of perfect conducting plate by plane wave illumination for different incident and scattering angles with co-polarization and cross-polarization are considered [6].

Cell size $\Delta d=1 \mathrm{~cm}$, time step $\Delta t=19.2 \mathrm{ps}$, problem space is $60 \times 60 \times 40$ cells, perfect conducting plate is $29 \times 29 \times 1$ cells located 9 cells above and parallel to the $x-y$ plane. Incident plane wave is Gaussian pulse with $1 \mathrm{kV} / \mathrm{m}$ amplitude, pulse width $\beta=64$ time steps. Use the default Liao absorption boundary condition.

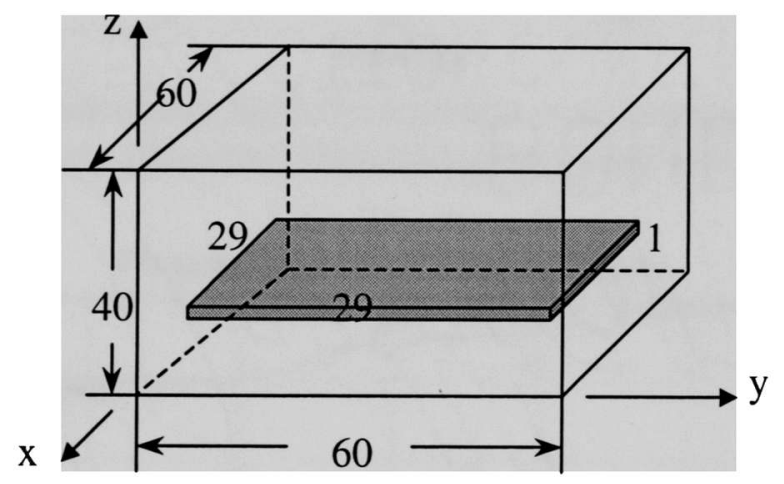

Figure 1.8 Geometry of the perfect conducting plate scatterer inside the problem space 
(1) Backscatter for normal incidence form $+z$ direction $\left(\phi=0^{\circ}, \theta=0^{\circ}\right)$

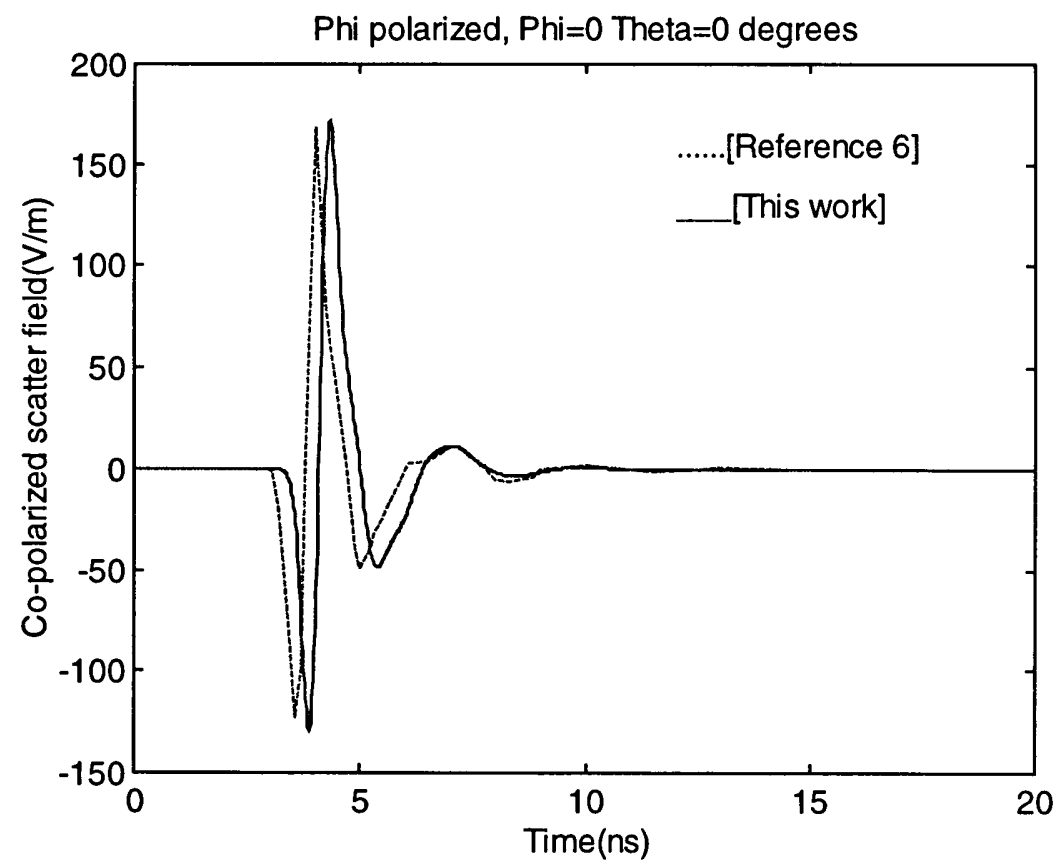

Figure 1.9 Far zone backscatter field from perfect conducting plate with normally incident (from $+z$ direction) Gaussian pulse plane wave by FDTD

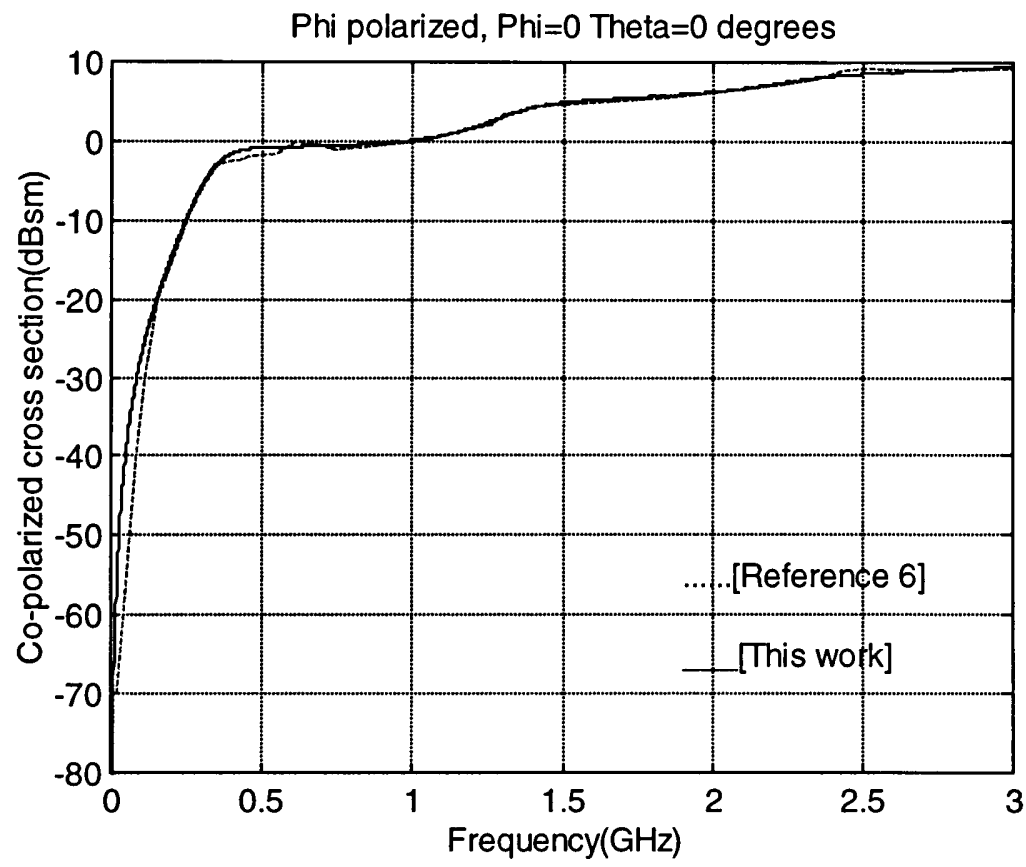

Figure 1.10 Far zone backscatter radar cross section from perfect conducting plate with normally incident Gaussian pulse plane wave 
(2) Backscatter for oblique incidence $\left(\phi=30^{\circ}, \theta=45^{\circ}\right)$

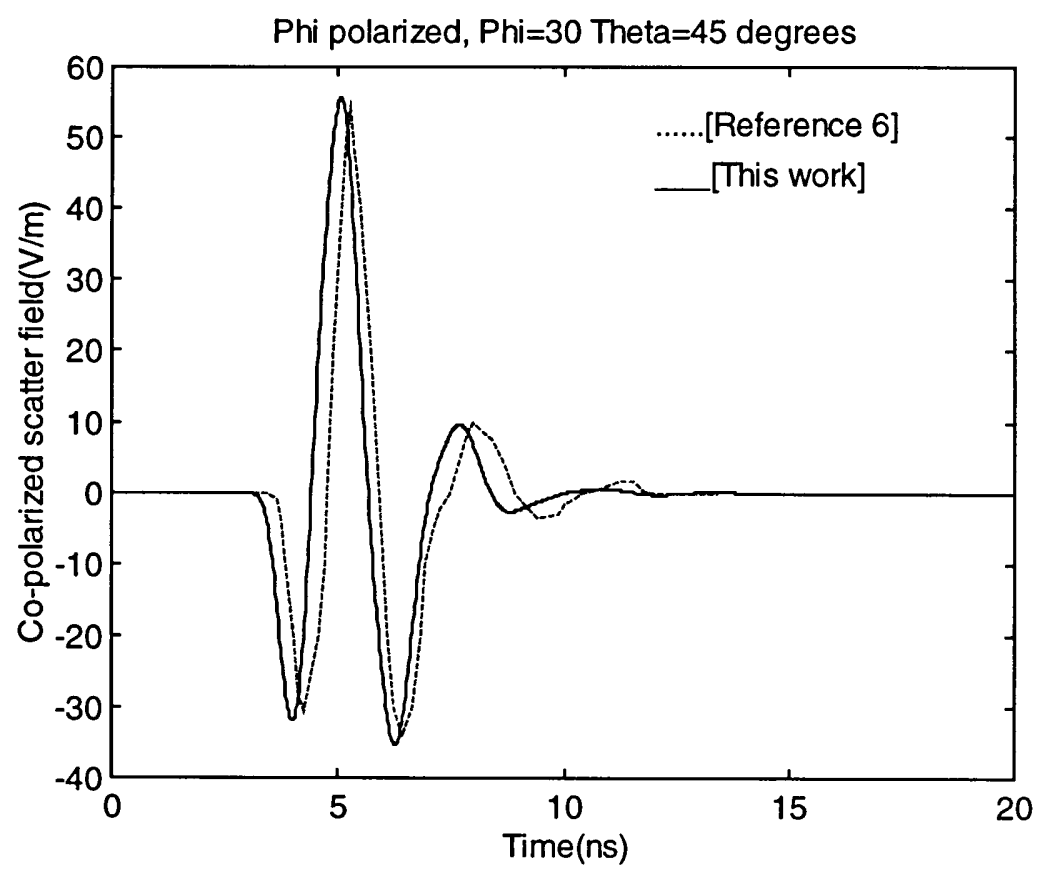

Figure 1.11 Far zone co-polarized backscatter field for perfect conducting plate with $\phi$ polarized Gaussian pulse plane wave incident from $\phi=30^{\circ}, \theta=45^{\circ}$ by FDTD

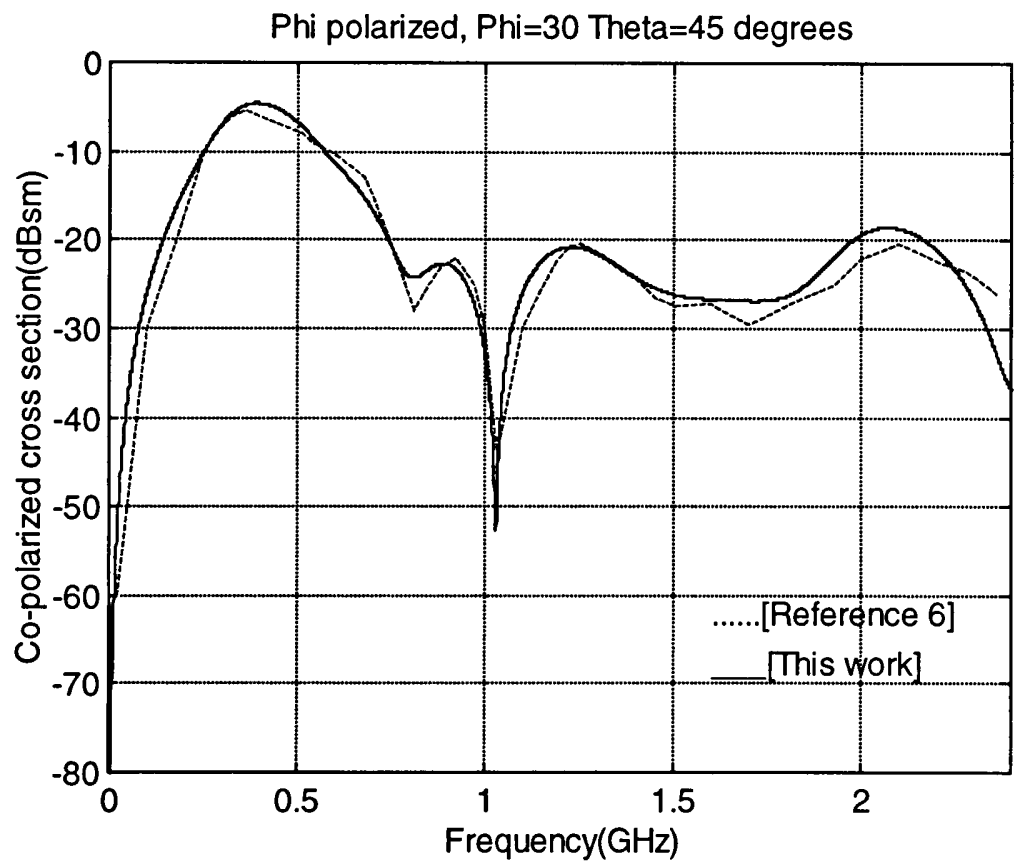

Figure 1.12 Far zone co-polarized backscatter radar cross section from perfect conducting plate 


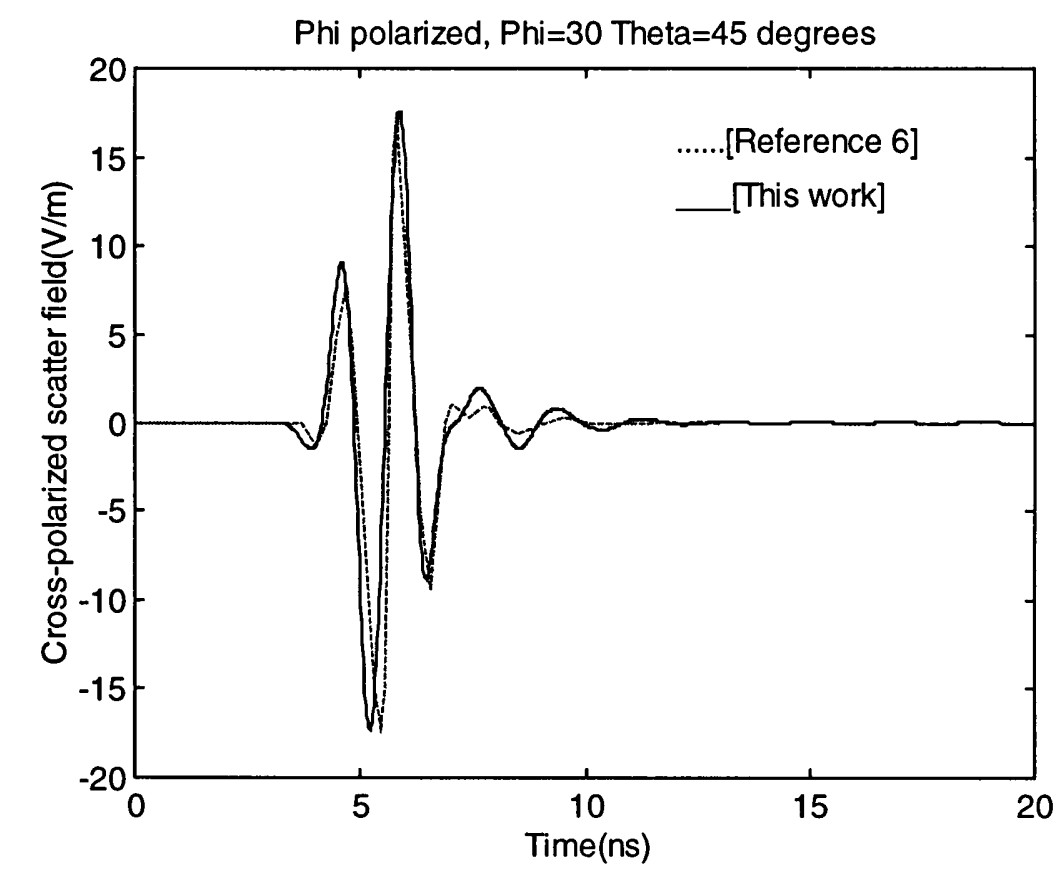

Figure 1.13 Far zone cross-polarized backscatter field for perfect conducting plate with $\phi$-polarized Gaussian pulse plane wave incident from $\phi=30^{\circ}, \theta=45^{\circ}$ by FDTD

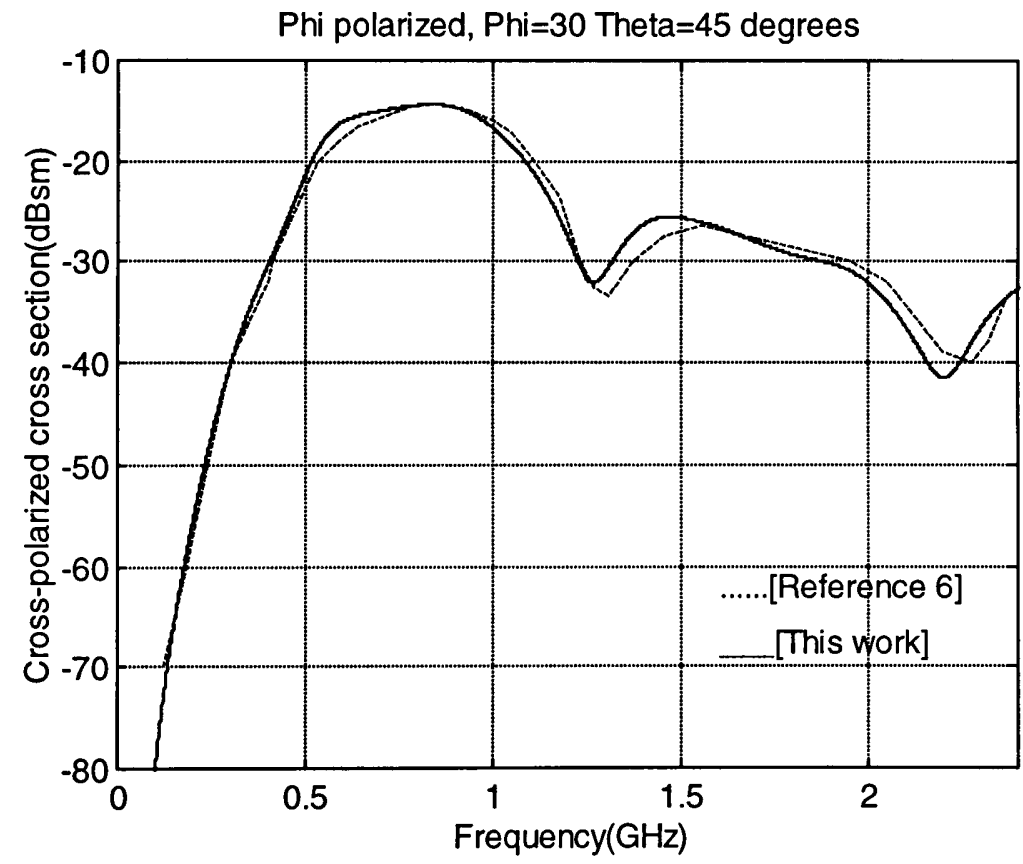

Figure 1.14 Far zone cross-polarized backscatter radar cross section from perfect conducting plate 


\section{Chapter 2}

\section{Validation of Diffraction Coefficients from Electromagnetic Software}

FDTD method applies numerical approach to compute scattering and radiation fields inside the problem space. Use computer simulation to model the object shape and define the parameters for research of interest.

\subsection{Diffraction Field Calculation}

The object will generate propagation waves depending on the medium inside and around it. Corners and edges of the structure can create diffraction; Faces of the objects and absorbing boundary of the problem space would create multiple reflection and refraction.

Diffraction is important for communications between building canyons in urban wireless environment. Different incident and diffraction directions could happen in 3dimensional and 2-dimensional diffraction. These data are useful for desire of terminal and channel facilities.

To get diffraction coefficients from building wedges like roofs or edges, we can obtain time domain diffraction fields through FDTD method. All possible waves will reach the receiver after the short modulated Gaussian pulse illumination containing wide frequency band information. Preliminary calculation is needed to confirm a long enough time range that only the concerned wedge diffraction field exists. Other scattered fields are desired outside the gating time to protect the diffraction field. 


\subsection{Diffraction Coefficients Validation of PEC Wedge}

Extract the diffraction field from the whole time domain calculation and use fast Fourier transform for both incident and diffraction pulses we have the frequency domain diffraction coefficients in wideband. Although diffraction is polarization sensitive same structure and processing are applied for the cross polarization components. We can compose the receiving fields from the cross-polarized directions according to the diffraction coefficients if the characteristic of the incident field is known.

Uniform Theory of Diffraction is the analytical solution to validate the diffraction coefficients of PEC wedge from FDTD method. It's the uniform expression for any diffraction angles around the wedge without singularity problem.

\subsection{Diffraction Coefficients of Building Wedges}

The agreements of analytical and numerical methods of PEC wedges are the foundation to extend the calculations for building material wedges by using FDTD method which is not available from asymptotic expressions. Although the building height and length are large scale, if the desire of the structure inside FDTD computing space can separate the diffraction fields with high accuracy from corner diffraction at the ends of the wedge and other possible scattering fields it doesn't need to model the real building wedge geometry. This study deals primarily in diffraction in the illuminated region.

Buildings are made of conductor and dielectric materials. Permittivity and conductivity for the building structural composition medium are variable parameters in FDTD calculation. 


\subsection{Experimental Validation}

Experimental measurement is another method to validate the diffraction coefficients of the measured wedge type. The receiving field is measured in real condition so that the diffraction coefficients would be more practical and accurate. 


\section{Chapter 3}

\section{3-Dimensional Diffraction Coefficient}

\subsection{Diffraction Phenomenon}

In wireless communication environment, signal is propagating under the shadowing of the buildings because of diffraction phenomenon that acts as an important component in the urban communication mechanism [12].
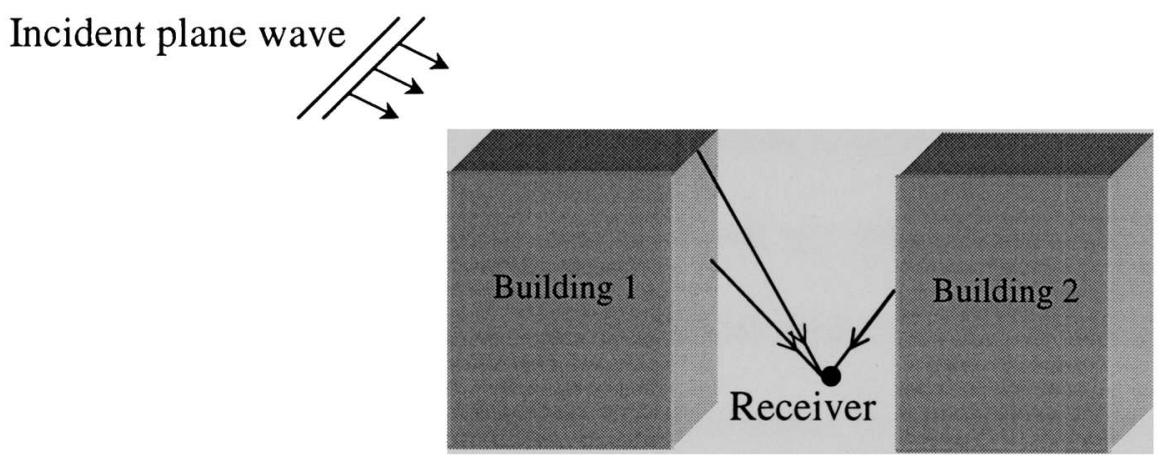

Figure 3.1 Diffraction around buildings

Diffraction is caused by the discontinuities of the surface or wedge where the incident electromagnetic wave met. It can be explained by the Huygen's principle:

Each point on a primary wavefront can be considered to be a point source for the production of secondary wavelets and that these wavelets combine to produce a new wavefront in the direction of propagation.

Although the received signal strength decreases as the receiver moves into the shadowed region without line of sight, only the diffracted fields will exist and usually have sufficient strength to produce a useful signal. This study primarily considers the illuminated region [14]. 


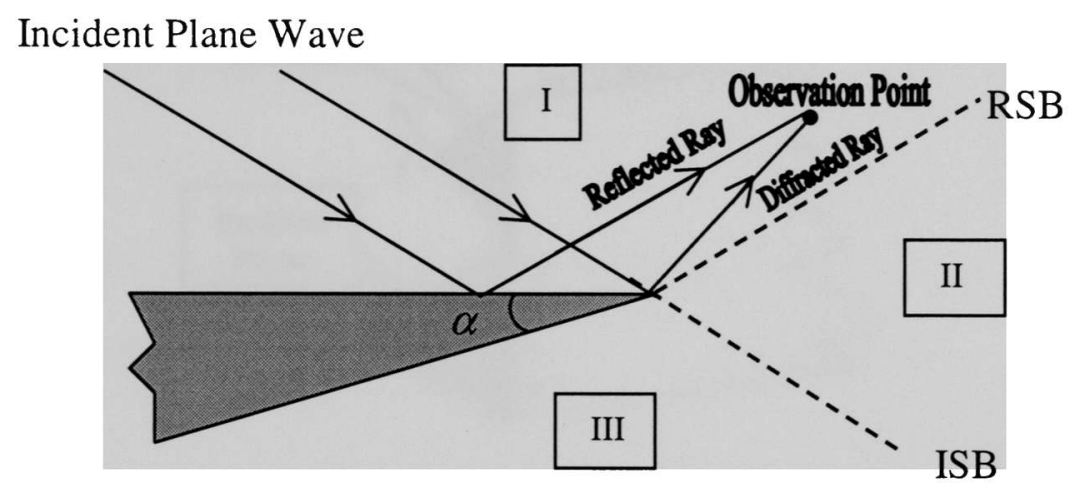

Figure 3.2 Shadow regions and boundaries caused by diffraction

The region illuminated by the incident field is referred to as the incident lit region, otherwise is the incident shadow region which are separated by the Incident Shadow Boundary (ISB). The reflected lit region and reflected shadow region are separated by the Reflection Shadow Boundary (RSB). The diffraction fields are located around the wedge in all regions.

$$
E^{t}=\left\{\begin{array}{cc}
E^{i}+E^{r}+E^{d} & \text { Region I } \\
E^{i}+E^{d} & \text { Region II } \\
E^{d} & \text { Region III }
\end{array}\right.
$$

$E^{t}$ is the total field, $E^{i}$ is the incident field, $E^{r}$ is the reflection field and $E^{d}$ is the diffraction field.

\subsection{Coordinate System}

\subsubsection{Edge-fixed Coordinate System}

Assume a plane wave is obliquely incident on a wedge, 


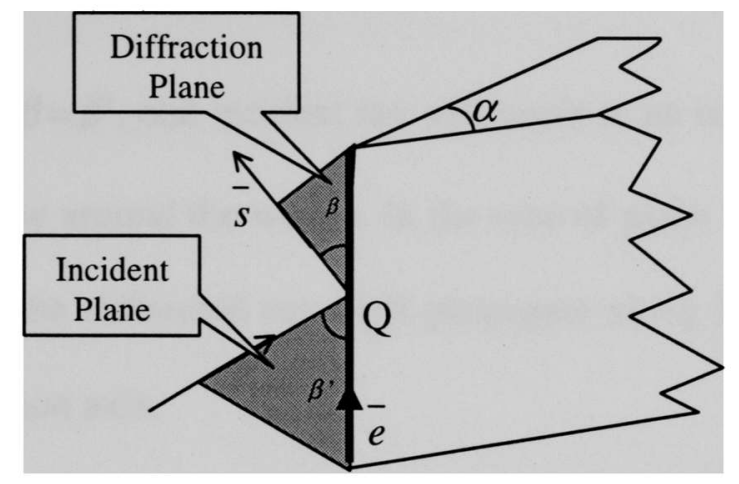

Figure 3.3 Edge-fixed coordinate system

$\bar{e}$ is the vector tangential to the wedge, $\bar{s}$ is the direction vector of the incident plane wave, $\beta$ ' is the angle between the source ray and the wedge, $\mathrm{Q}$ is the diffraction point on the wedge, $\bar{s}$ is the diffracted ray vector to the receiver, $\beta$ is the angle between the diffracted ray and the wedge. The edge and the incident wave form the incident edgefixed plane. The edge and the diffraction wave form the diffraction edge-fixed plane.

Keller's law of edge diffraction shows: A diffracted ray and the corresponding incident ray make equal angles with the edge at the point of diffraction, provided that they are in the same medium. They lie on the opposite sides of the plane normal to the edge at the point of diffraction.

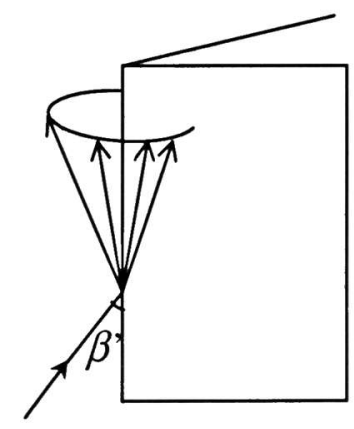

Figure 3.4 3-Dimensional diffraction cone 
This law states that $\beta=\beta$, one incident ray will result in an infinite number of diffracted rays lying on the cone around the wedge. In the case of plane wave oblique incidence on the straight wedge, the diffracted rays will propagate along the parallel cones with the wedge as their common axis.

\subsubsection{Ray-fixed Coordinate System}

Based on the edge-fixed coordinate system $\left(\bar{e}, \bar{s}, \overline{s^{\prime}}\right)$ we have ray-fixed coordinate system $(\bar{\beta}, \bar{\beta}, \bar{\phi}, \bar{\phi})$ which makes the diffraction coefficients simplify to two scalars: soft diffraction coefficient $D_{s}$ and hard diffraction coefficient $D_{h}$.

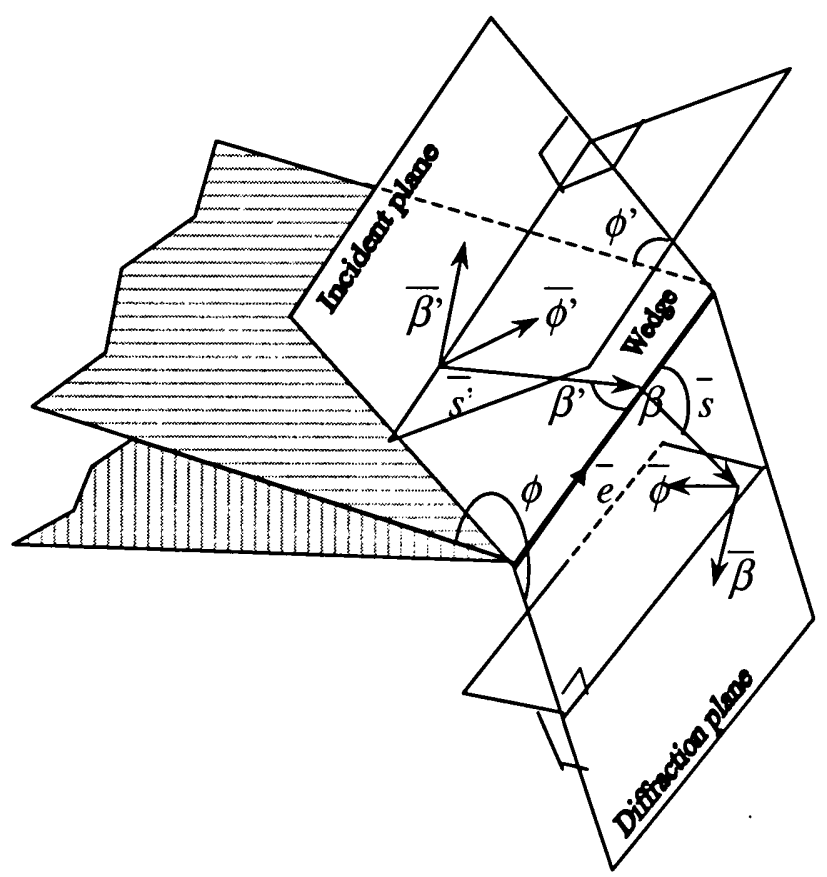

Figure 3.5 Ray-fixed coordinate system 
$\bar{s}$ represents the propagation direction of the incident wave which is resolved into two orthogonal polarized components $\bar{\beta}$, in the incident plane and $\bar{\phi}$, normal to the incident plane. $\bar{s}$ represents the propagation direction of the diffraction wave which is resolved into two orthogonal polarized components $\bar{\beta}$ in the diffraction plane and $\bar{\phi}$ normal to the diffraction plane. $\phi$ ' and $\phi$ are the angles between the surface of the wedge to the incident plane and diffraction plane respectively.

$$
\begin{gathered}
\overline{\beta^{\prime}}=\bar{\phi} \times \overline{s^{\prime}} \\
\bar{\beta}=\bar{\phi} \times \bar{s}
\end{gathered}
$$

The incident and diffraction fields can be resolved into two orthogonal components from the above definition

$$
\begin{aligned}
& E^{i}=E_{\beta}^{i}, \bar{\beta}, E_{\phi}^{i}, \bar{\phi} \\
& E^{d}=E_{\beta}^{d} \bar{\beta}+E_{\phi}^{d} \bar{\phi}
\end{aligned}
$$

\subsection{3-Dimensional Diffraction Coefficient of PEC Wedge}

Uniform Theory of Diffraction (UTD) has accurate solution for diffraction coefficients of PEC wedge without singularity problems but it's difficult to extend the analysis for wedge composed of dielectric and imperfect conductor like building materials. Numerical approach for diffraction coefficients using FDTD method is applied for PEC wedge first. The agreement to the asymptotic expressions for PEC wedge is the foundation to calculate diffraction coefficients of dielectric wedges. 


\subsubsection{Asymptotic 3-Dimensional Diffraction Coefficient of PEC Wedge}

The 3-dimensional diffraction coefficients of PEC wedge can be expressed by a 2 $\times 2$ matrix using ray-fixed coordinate system.

$$
E^{d}=E^{i} \cdot D \cdot A(s) \cdot e^{-j k s}
$$

$D$ is the dyadic diffraction coefficient

$$
D=-\bar{\beta}, \bar{\beta} \cdot D_{s}-\bar{\phi} \cdot \bar{\phi} \cdot D_{h}
$$

$E^{i}$ is the incident wave at the wedge, $E^{d}$ is the diffraction wave at the receiving point, $\mathrm{k}$ is the wavenumber of the medium, $s$ is the distance from the diffraction point on the wedge to the receiving point, $A(s)$ is the spreading factor

$$
A(s)=\sqrt{\frac{\rho}{s(s+\rho)}}
$$

$\rho$ is the edge caustic distance. For straight wedge and plane wave incidence, $\rho \rightarrow \infty$,

$$
\begin{gathered}
A(s)=\sqrt{\frac{1}{s}} \\
E_{\beta}^{d} \bar{\beta}+E_{\phi}^{d} \bar{\phi}=\left(E_{\beta}^{i}, \bar{\beta},+E_{\phi}^{i}, \bar{\phi}\right) \cdot\left(-\bar{\beta}, \bar{\beta} D_{s}-\bar{\phi}, \bar{\phi} D_{h}\right) \cdot \sqrt{\frac{1}{s}} \cdot e^{-j k s}
\end{gathered}
$$

From the correspondent quantities in $\bar{\beta}$ and $\bar{\phi}$,

$$
\begin{gathered}
E_{\beta}^{d}=-D_{s} E_{\beta}^{i} \cdot \sqrt{\frac{1}{s}} e^{-j k s} \\
E_{\phi}^{d}=-D_{h} E_{\phi}^{i} \cdot \sqrt{\frac{1}{s}} e^{-j k s} \\
{\left[\begin{array}{l}
E_{\beta}^{d} \\
E_{\phi}^{d}
\end{array}\right]=\left[\begin{array}{cc}
-D_{s} & 0 \\
0 & -D_{h}
\end{array}\right]\left[\begin{array}{l}
E_{\beta^{\prime}}^{i} \\
E_{\phi^{\prime}}^{i}
\end{array}\right] \cdot \sqrt{\frac{1}{s}} \cdot e^{-j k s}}
\end{gathered}
$$


$D_{s}$ and $D_{h}$ are scalar soft diffraction coefficient and hard diffraction coefficient when soft and hard boundary conditions are used. $D_{s}$ is related to the parallel component on the edge-fixed diffraction plane which is $\bar{\beta}$ polarized, while $D_{h}$ is related to the normal component to the edge-fixed diffraction plane which is $\bar{\phi}$ polarized.

Asymptotic diffraction coefficients of PEC wedge to make total fields continuous at the incident and reflection boundaries are $[7,8]$

$$
\begin{aligned}
D_{s}=\frac{-e^{-j \pi / 4}}{2 n \sqrt{2 \pi k} \sin \beta} & \left\{\cot \left[\frac{\pi+\left(\phi-\phi^{\prime}\right)}{2 n}\right] F\left[k L^{i} a^{+}\left(\phi-\phi^{\prime}\right)\right]\right. \\
& +\cot \left[\frac{\pi-\left(\phi-\phi^{\prime}\right)}{2 n}\right] F\left[k L^{i} a^{-}\left(\phi-\phi^{\prime}\right)\right] \\
& -\cot \left[\frac{\pi+\left(\phi+\phi^{\prime}\right)}{2 n}\right] F\left[k L^{m} a^{+}\left(\phi+\phi^{\prime}\right)\right] \\
& \left.-\cot \left[\frac{\pi-\left(\phi+\phi^{\prime}\right)}{2 n}\right] F\left[k L^{r o} a^{-}\left(\phi+\phi^{\prime}\right)\right]\right\} \\
D_{h}=\frac{-e^{-j \pi / 4}}{2 n \sqrt{2 \pi k} \sin \beta} & \left\{\cot \left[\frac{\pi+\left(\phi-\phi^{\prime}\right)}{2 n}\right] F\left[k L^{i} a^{+}\left(\phi-\phi^{\prime}\right)\right]\right. \\
& +\cot \left[\frac{\pi-\left(\phi-\phi^{\prime}\right)}{2 n}\right] F\left[k L^{i} a^{-}\left(\phi-\phi^{\prime}\right)\right] \\
& +\cot \left[\frac{\pi+\left(\phi+\phi^{\prime}\right)}{2 n}\right] F\left[k L^{r n} a^{+}\left(\phi+\phi^{\prime}\right)\right] \\
& \left.+\cot \left[\frac{\pi-\left(\phi+\phi^{\prime}\right)}{2 n}\right] F\left[k L^{r o} a^{-}\left(\phi+\phi^{\prime}\right)\right]\right\}
\end{aligned}
$$


Because plane wave incidence will have cylindrical wavefront for all diffraction rays from straight wedge, distance parameters will be reduced to

$$
L^{i}=L^{m}=L^{r o}=s \cdot \sin ^{2} \beta
$$

Associated function

$$
a^{ \pm}(\gamma)=2 \cos ^{2}\left(\frac{2 n \pi N^{ \pm}-\gamma}{2}\right)
$$

The interior angle of wedge $\alpha=(2-n) \cdot \pi$ and the exterior angle is $n \pi$. Use right angle wedge to simulate general building wedges like the edges and roofs, apply $\alpha=90^{\circ}$, $n=\frac{3}{2}$.

$N^{ \pm}$is the integer which is most closely satisfied

$$
\begin{gathered}
2 n \pi N^{ \pm}-\gamma= \pm \pi \\
\gamma=\phi \pm \phi
\end{gathered}
$$

$$
0 \leq \phi^{\prime} \leq \pi, 0 \leq \phi \leq 2 \pi,-\pi \leq \gamma=\phi \pm \phi^{\prime} \leq 3 \pi
$$

$$
N^{+}= \begin{cases}0 & \gamma<\frac{\pi}{2} \\ 1 & \gamma \geq \frac{\pi}{2}\end{cases}
$$

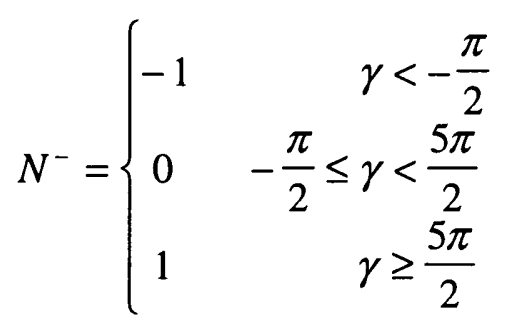

and when

$$
\phi= \begin{cases}\phi^{\prime}-\pi & N^{+}=0 \\ 2 \pi-\phi^{\prime} & N^{+}=1 \\ \pi \pm \phi^{\prime} & N^{-}=0\end{cases}
$$


Transition function $\quad F(x)=2 j \sqrt{x} e^{j x} \int_{\sqrt{x}}^{\infty} e^{-j t^{2}} d t$

$$
\begin{aligned}
& F(x)= \begin{cases}2 j \sqrt{x} e^{j x} \sqrt{\frac{\pi}{2}}\left[\frac{1}{2}-c(\sqrt{x})-j\left(\frac{1}{2}-s(\sqrt{x})\right)\right] & x<100 \\
1+\frac{j}{2 x}-\frac{3}{4 x^{2}}-\frac{15 j}{8 x^{3}}+\frac{75}{16 x^{4}} & x \geq 100\end{cases} \\
& c(x)=\sqrt{\frac{2}{\pi}} \int_{0}^{x} \cos \left(t^{2}\right) d t \\
& s(x)=\sqrt{\frac{2}{\pi}} \int_{0}^{x} \sin \left(t^{2}\right) d t
\end{aligned}
$$

If the characteristic of the incident wave is known, we can have the $\bar{\beta}$ and $\bar{\phi}$ polarized fields by $D_{s}$ and $D_{h}$ using above equations and composite them for the diffraction electric field at the receiving end according to the ray-fixed coordinate. The quantity relationship between $\bar{\beta}$ and $\bar{\phi}$ polarization is usually changed so that the polarization property of the diffraction ray will be different from the incident ray.

\subsubsection{3-Dimensional Diffraction Coefficient of PEC Wedge Using FDTD Method}

Diffraction coefficient can be found numerically by using FDTD method from equations (2.3) and (2.6) for PEC wedge in frequency domain [10].

$$
D=\frac{f f t\left(E^{d}\right)}{f f t\left(E^{i}\right)} \cdot \sqrt{s} \cdot e^{j k s}
$$

Illuminate the wedge with short pulse. Sample the fields at the observation point according to the distance and direction and extract the diffraction wave from all other field responses using time-gating. The Fourier transform gives the diffraction coefficient over a wide range of frequency band. 
To find the diffraction coefficients including frequency components at $850 \mathrm{MHz}$ and $1.7 \mathrm{GHz}$ in $1.06 \mathrm{~m}$ distance around the wedge for oblique incidence of $\beta^{\prime}=70^{\circ}$, $\phi^{\prime}=150^{\circ}$ to right angle PEC wedge, define cell size $\Delta x=\Delta y=\Delta z=\Delta d=1.41 \mathrm{~cm}$. The valid frequency band will be $f_{\max }=2.12 \mathrm{GHz}$ although it will be more accurate at $850 \mathrm{MHz}\left(\frac{\lambda}{\Delta d}=25\right)$ than at $1.7 \mathrm{GHz}\left(\frac{\lambda}{\Delta d}=12.5\right)$. The diffraction angles are $35^{\circ}, 40^{\circ}$, $45^{\circ}, 50^{\circ}, 60^{\circ}, 70^{\circ}, 80^{\circ}$ and $100^{\circ}$ around the wedge [9].

We have known that the modulated Gaussian pulse plane wave at modulated frequency $f_{0}=850 \mathrm{MHz}$ with time step $\Delta t=27.188 \mathrm{ps}$ and pulse width $\beta=32$ time steps will have high enough energy at both $850 \mathrm{MHz}$ and $1.7 \mathrm{GHz}$. In case of soft diffraction coefficient $D_{s}$, only field strength of diffraction ray at $\bar{\beta}$ direction and incident ray at $\bar{\beta}$ direction are concerned. In case of hard diffraction coefficient $D_{h}$, only field strength of diffraction ray at $\bar{\phi}$ direction and incident ray at $\bar{\phi}$, direction are concerned. Let the incident plane wave in $\bar{\beta}$, or $\bar{\phi}$, polarization for $D_{s}$ or $D_{h}$ respectively. If the wedge is set parallel to the z-axis, $\bar{\beta}$, and $\bar{\phi}$ in ray-fixed coordinate system will be parallel or opposite to $\theta$ or $\phi$ polarization definition in spherical system so that it will be convenient to specify the polarization of the incident plane wave. 


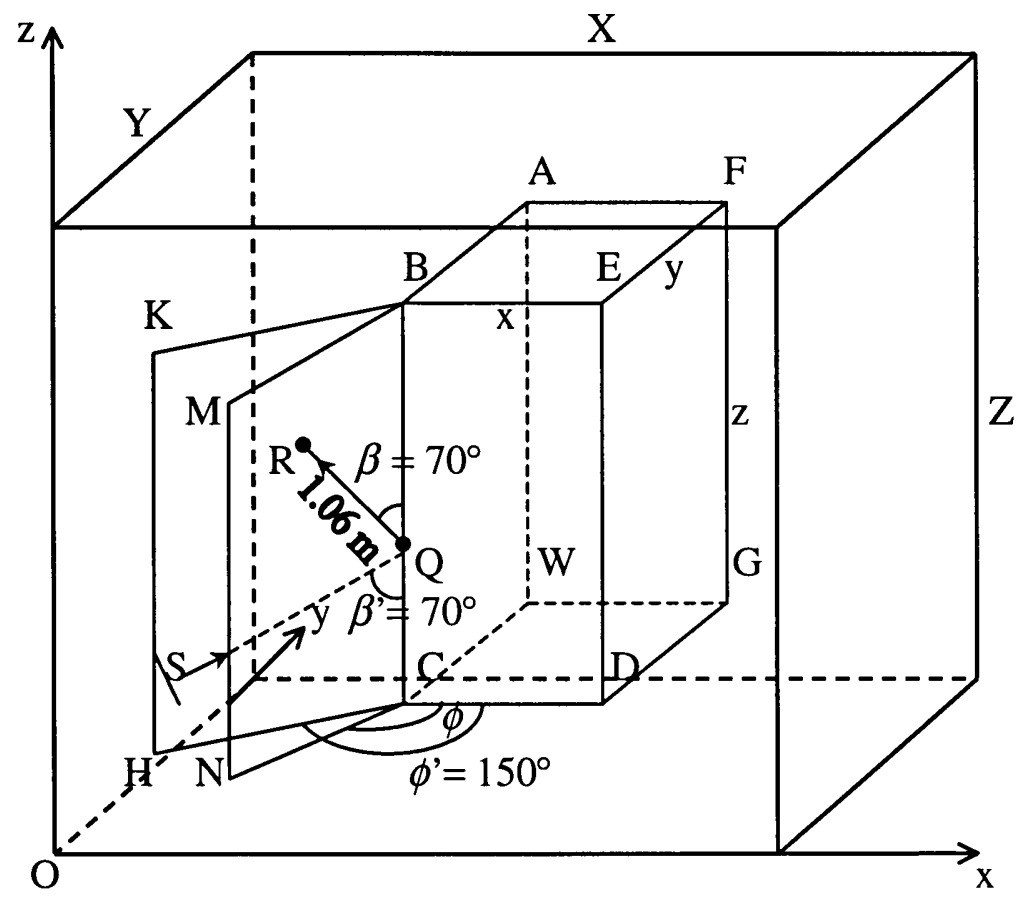

Figure 3.6 Geometry of the structure inside problem space to get diffraction coefficients from $\phi^{\prime}=150^{\circ}, \beta^{\prime}=70^{\circ}$ plane wave incidence

The cell numbers of the problem space are $\mathrm{X}, \mathrm{Y}$ and $\mathrm{Z}$, the cell numbers of the wedge structure are $\mathrm{x}, \mathrm{y}$ and $\mathrm{z}$. PEC right angle wedge $\mathrm{BC}$ is formed by surface planes BCDE and $\mathrm{ABCW}$. $\mathrm{Q}$ is the point of diffraction on the wedge $\mathrm{BC}$. Incident ray $\mathrm{SQ}$ is on the incident plane BKHC. Observation point $\mathrm{R}$ and diffraction ray $\mathrm{QR}$ are on the diffraction plane BMNC. $\phi^{\prime}=\angle \mathrm{HCD}=150^{\circ}$ is the incident angle between the incident plane BKHC and wedge surface plane BCDE. $\beta^{\prime}=\angle \mathrm{SQC}=70^{\circ}$ is the angle between the incident ray SQ and the wedge $\mathrm{BC}$.

$$
\begin{aligned}
& \phi_{f d t d}^{i}=210^{\circ} \\
& \theta_{f d t d}^{i}=110^{\circ}
\end{aligned}
$$

are direction of the incident plane wave $S$ defined in FDTD. 
$\phi=\angle \mathrm{NCD}=35^{\circ}, 40^{\circ}, 45^{\circ}, 50^{\circ}, 60^{\circ}, 70^{\circ}, 80^{\circ}, 100^{\circ}$ is the diffraction angle between the diffraction plane $\mathrm{BMNC}$ and wedge surface plane $\mathrm{BCDE}$. $\beta=\angle \mathrm{RQB}=70^{\circ}$ is the angle between the diffraction ray $\mathrm{QR}$ and the wedge $\mathrm{BC}$. Observation point $\mathrm{R}$ is in the distance of $|\mathrm{RQ}|=1.06 \mathrm{~m}$ from the diffraction point $\mathrm{Q}$. Absorbing boundary is Liao.
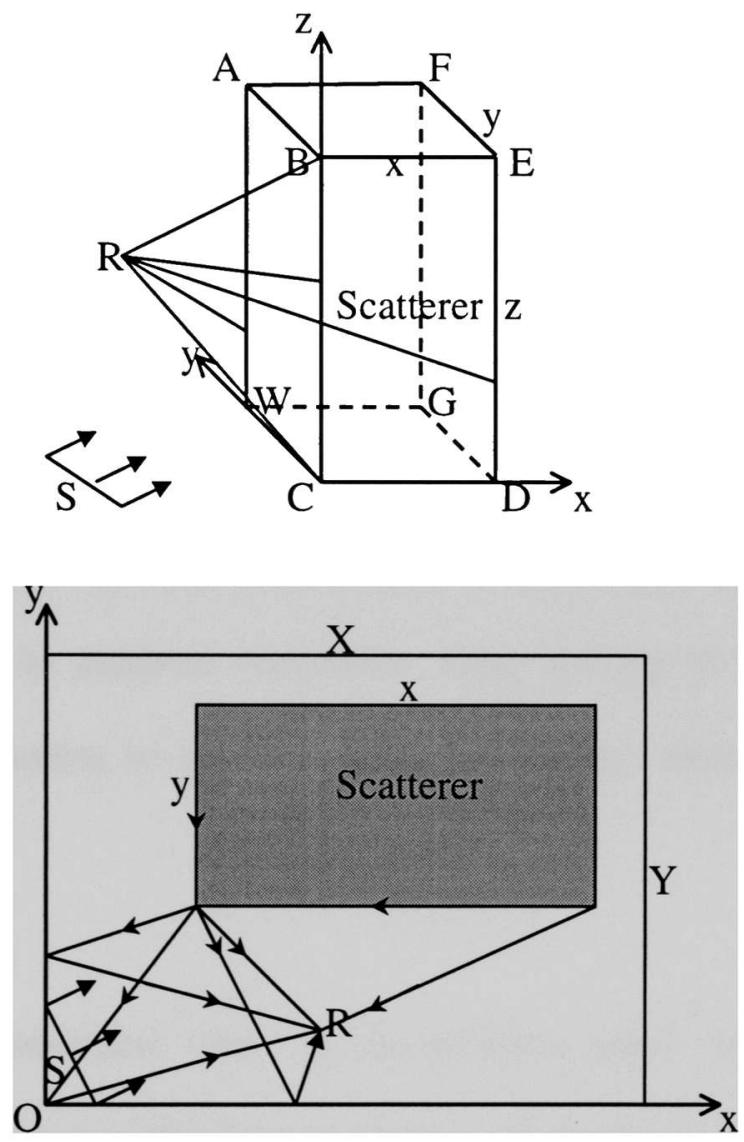

Figure 3.7 Scattered Fields Consideration from side view and top view

The diffraction from wedge $\mathrm{BC}$ is the desired pulse, but there are other pulses to the observation point R. (1) To separate the diffraction from wedge ED, x could not be small. (2) To separate the diffraction from wedge AW or multiple diffraction from wedge AW and $\mathrm{BC}$ (for some diffraction angles), y needs to be large enough. (3) To separate the 
diffraction from corner $\mathrm{B}$ and $\mathrm{C}$, the height between $\mathrm{BR}$ and $\mathrm{CR}$ should be large enough. Because the incident wave is from low to high $\mathrm{R}$ is not set in the middle of $\mathrm{z}$ but a little higher to let top and bottom diffraction waves get to $\mathrm{R}$ almost at the same time. Based on the ray solution from UTD, if the wedge diffraction field is separate from the corner diffraction at the end of the wedge, the height of the structure is not necessary to be as the whole building. (4) To avoid reflections from the walls parallel to ABCW and BCDE and corner close to wedge $\mathrm{BC}$ of the absorbing boundary, put more enough free space between the structure and the boundary for attenuation, time separation and better absorption of boundary reflection. Other diffraction or reflection waves from corners, wedges and surfaces of the structure and the boundary will come behind the above waves. Satisfying these will take much memory, so every dimension of the structure and problem space needs to be assigned reasonable. After making all other scattered fields behind, the first pulse access to the receiver $R$ will be the diffraction field from the desired wedge $\mathrm{BC}$.

Above are 3-dimensional views of the problem space and the structure. The following geometry figures are all of top views for simplicity to show the cell numbers inside and around the structure. 10 cells free space from the top and bottom surface of the structure to the boundary are common for all 2-dimensional figures. Each geometry is used for both soft and hard diffraction coefficients only the polarization of the fields is different. 
3.3.2.1 Diffraction Coefficients of PEC Wedge for $\phi^{\prime}=150^{\circ}, \beta^{\prime}=70^{\circ}, \phi=35^{\circ}$

1) Soft Diffraction Coefficient $D_{s}$ for $\phi=35^{\circ}$

$$
\begin{aligned}
& X=153 \\
& Y=125 \\
& Z=280 \\
& X=108 \\
& y=40 \\
& z=260 \\
& R(93,35,186)
\end{aligned}
$$

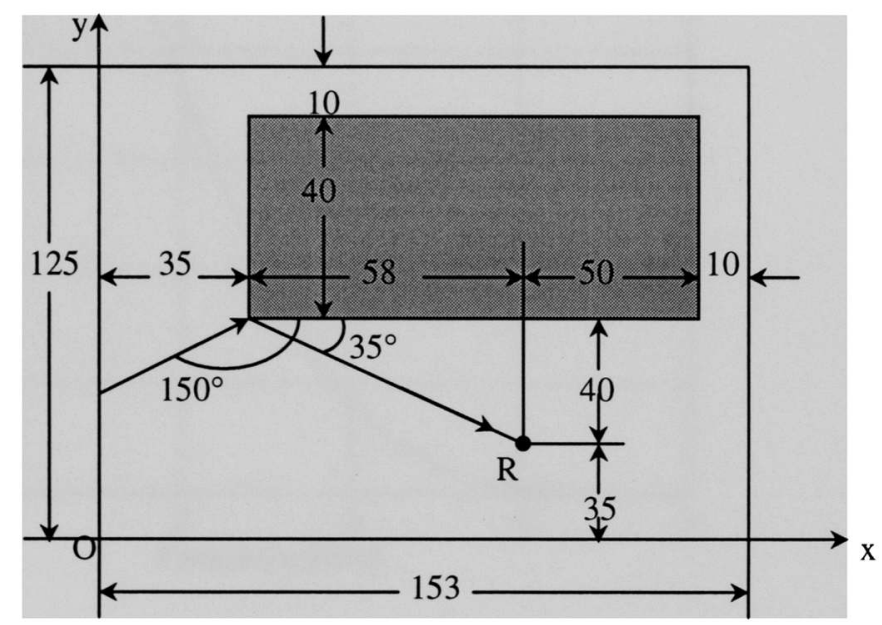

Figure 3.8 Geometry of structure and problem space to get the diffraction coefficient for $\phi^{\prime}=150^{\circ}, \beta^{\prime}=70^{\circ}$ and $\phi=35^{\circ}$ in $\mathrm{R}=1.06 \mathrm{~m}$

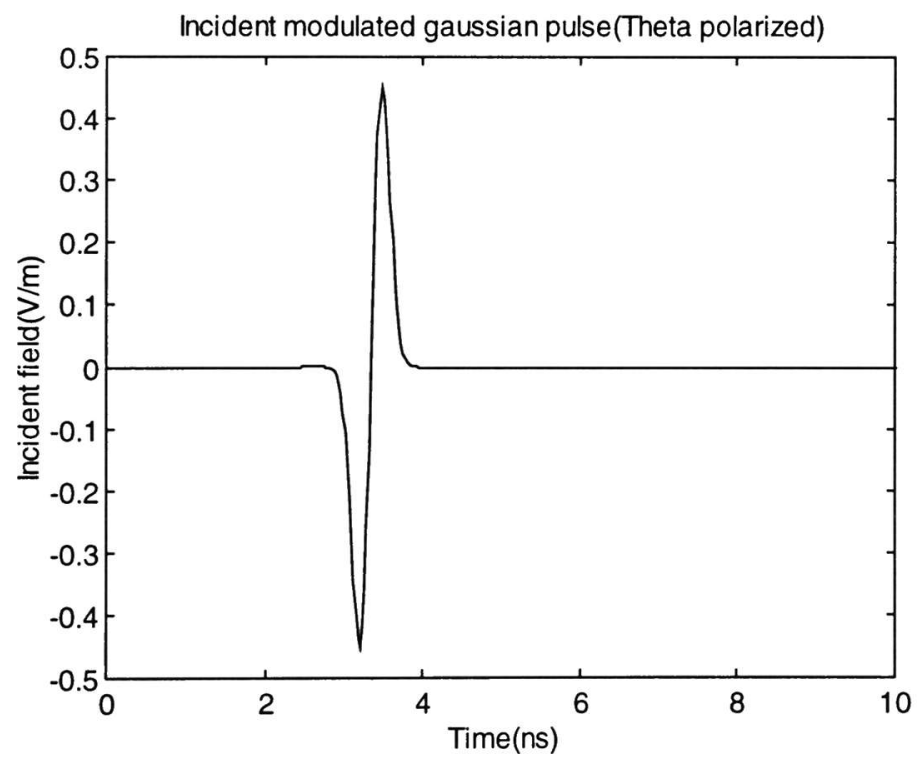

Figure $3.9 \theta$-polarized incident modulated Gaussian plane wave inside the problem space from $\phi_{\text {ftrd }}^{i}=210^{\circ} \theta_{\text {fttd }}^{i}=110^{\circ}$ ( $f_{0}=850 \mathrm{MHz}$, pulse width $\beta=32$ time steps, $\Delta t=27.188 \mathrm{ps}, \mathrm{A}=1 \mathrm{~V} / \mathrm{m}$ ) 


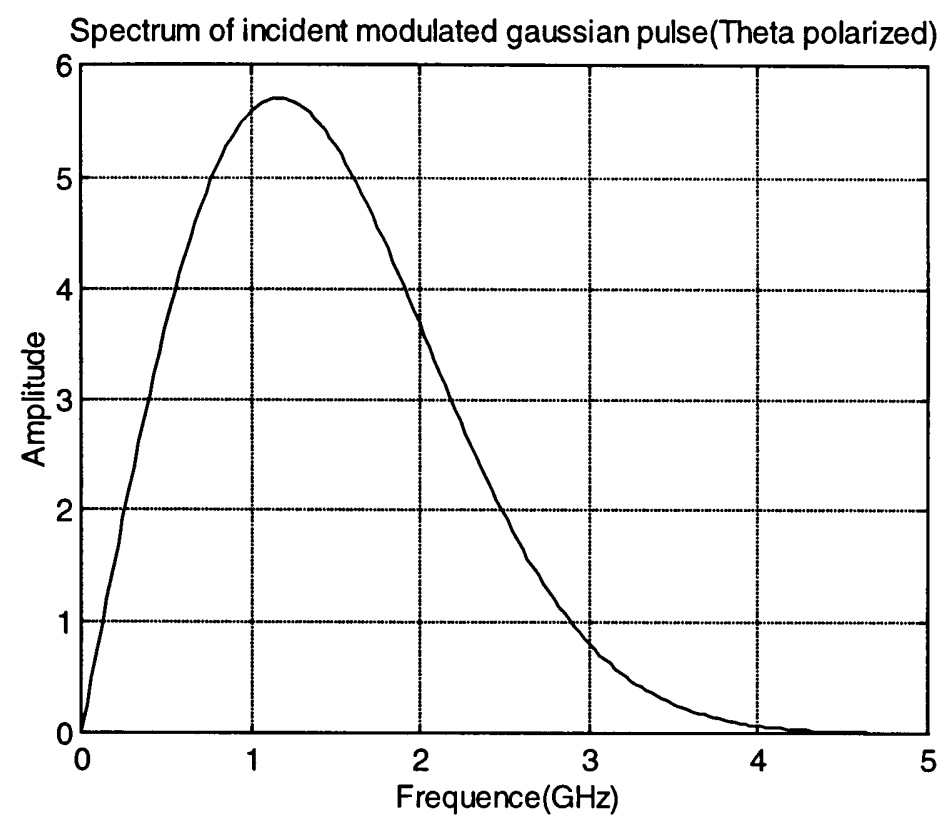

Figure 3.10 Spectrum of the $\theta$-polarized incident modulated Gaussian plane wave

Save time domain waveforms $E_{x}, E_{y}$ and $E_{z}$ at the observation point R.

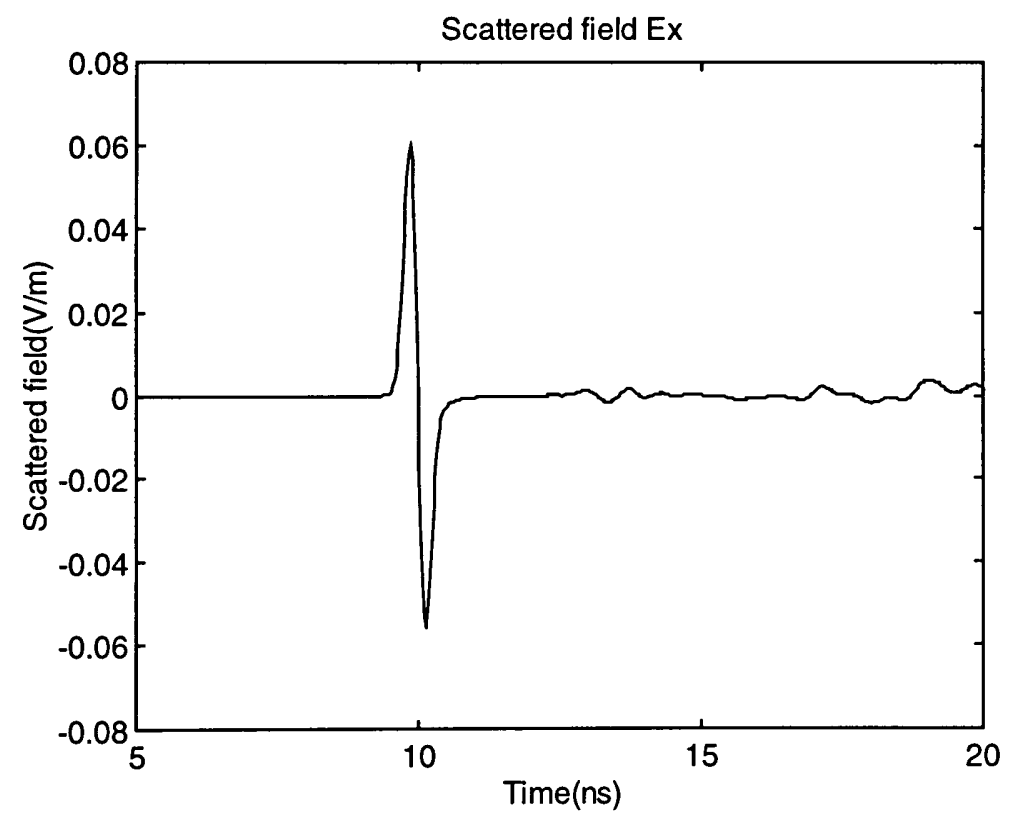

Figure 3.11 Scattered field Ex at receiver from $\theta$-polarized plane wave incident on PEC wedge for $\phi=35^{\circ}$ 


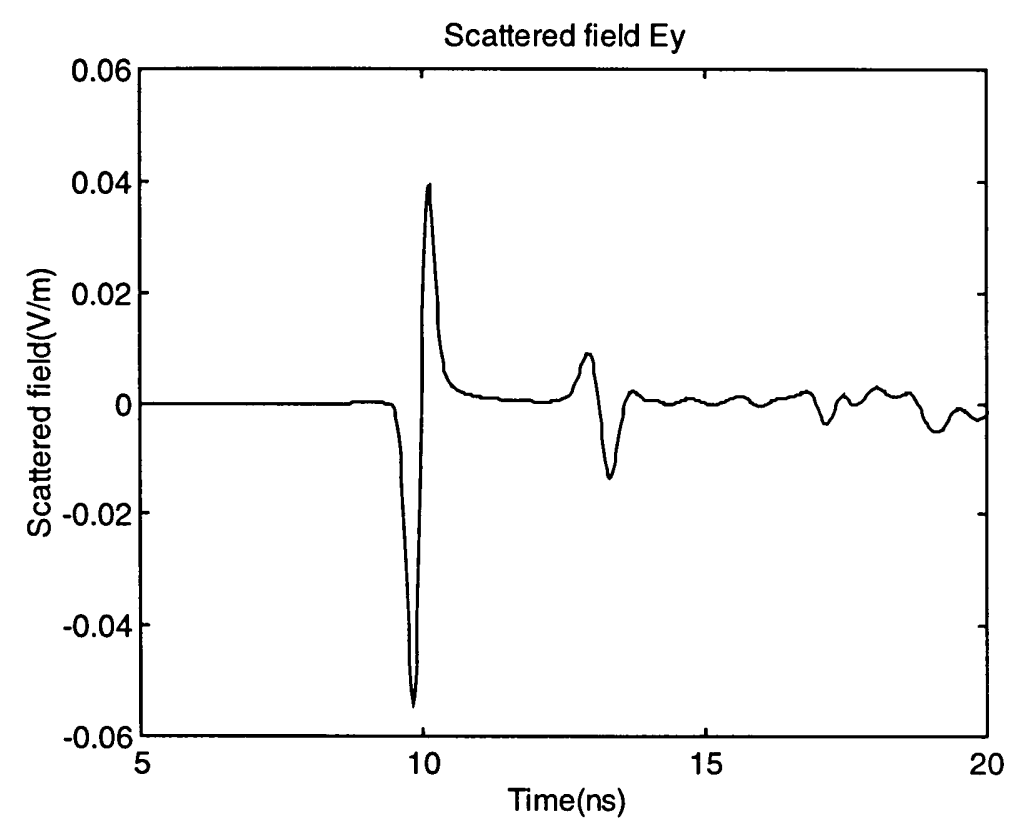

Figure 3.12 Scattered field Ey at receiver from $\theta$-polarized plane wave incident on PEC wedge for $\phi=35^{\circ}$

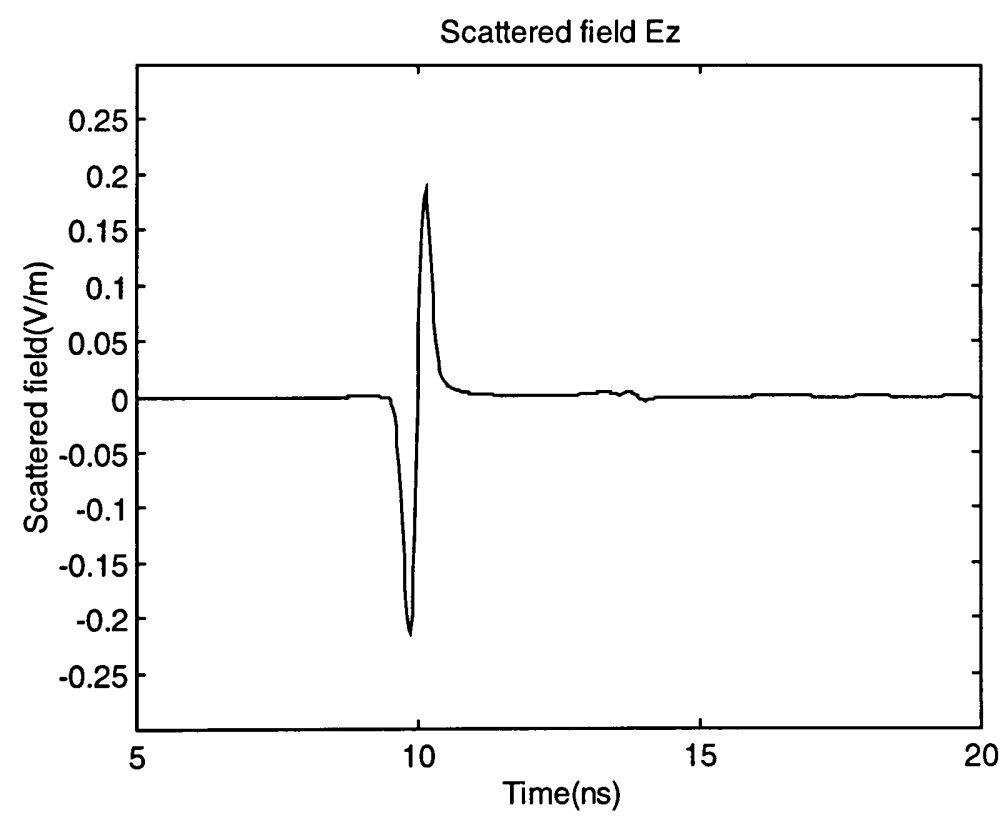

Figure 3.13 Scattered field $\mathrm{Ez}$ at receiver from $\theta$-polarized plane wave incident on PEC wedge for $\phi=35^{\circ}$ 
Use coordinate transformation from Cartesian to spherical system [13]

$$
E_{\theta}=E_{x} \cos \theta \cos \phi+E_{y} \cos \theta \sin \phi-E_{z} \sin \theta
$$

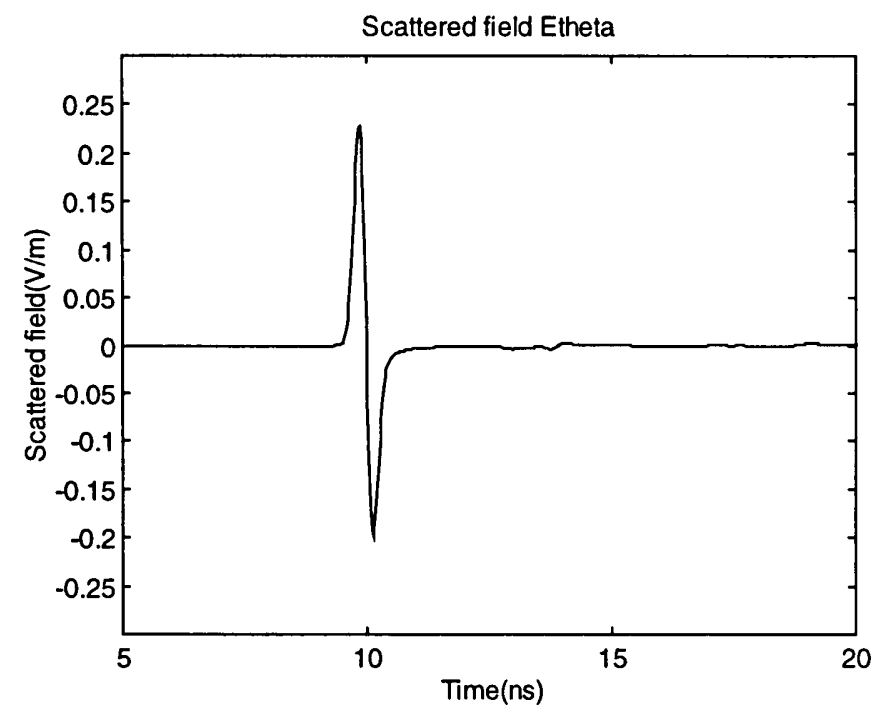

Figure 3.14 Scattered field at receiver from $\theta$-polarized plane wave incident on PEC wedge for $\phi=35^{\circ}$

Use time-gating to get the first pulse as the desired diffraction field from the wedge.

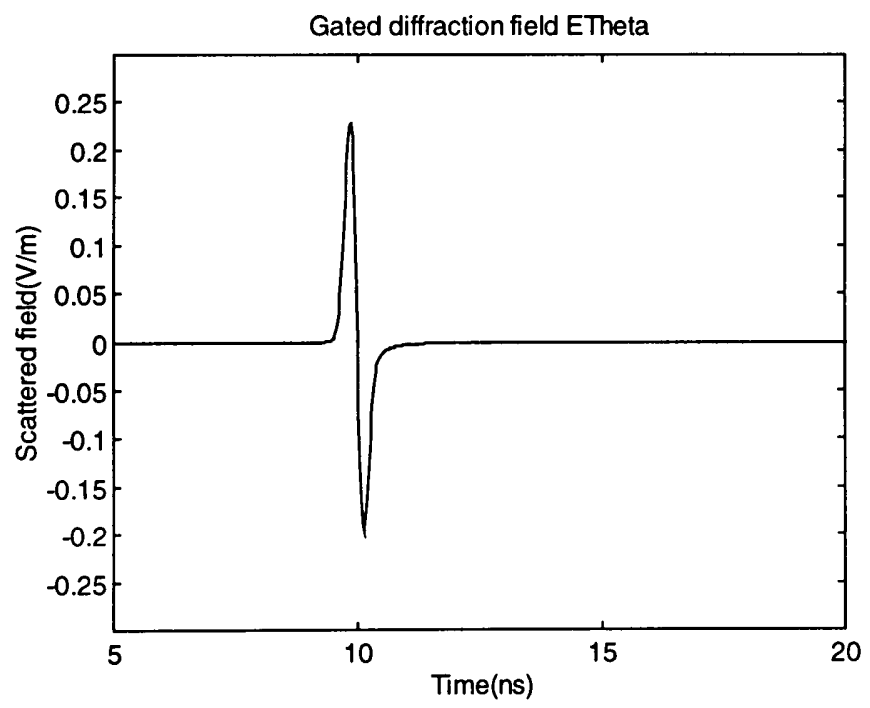

Figure 3.15 Extract the diffraction field from $\theta$-polarized plane wave incident on PEC wedge for $\phi=35^{\circ}$ using time-gating 


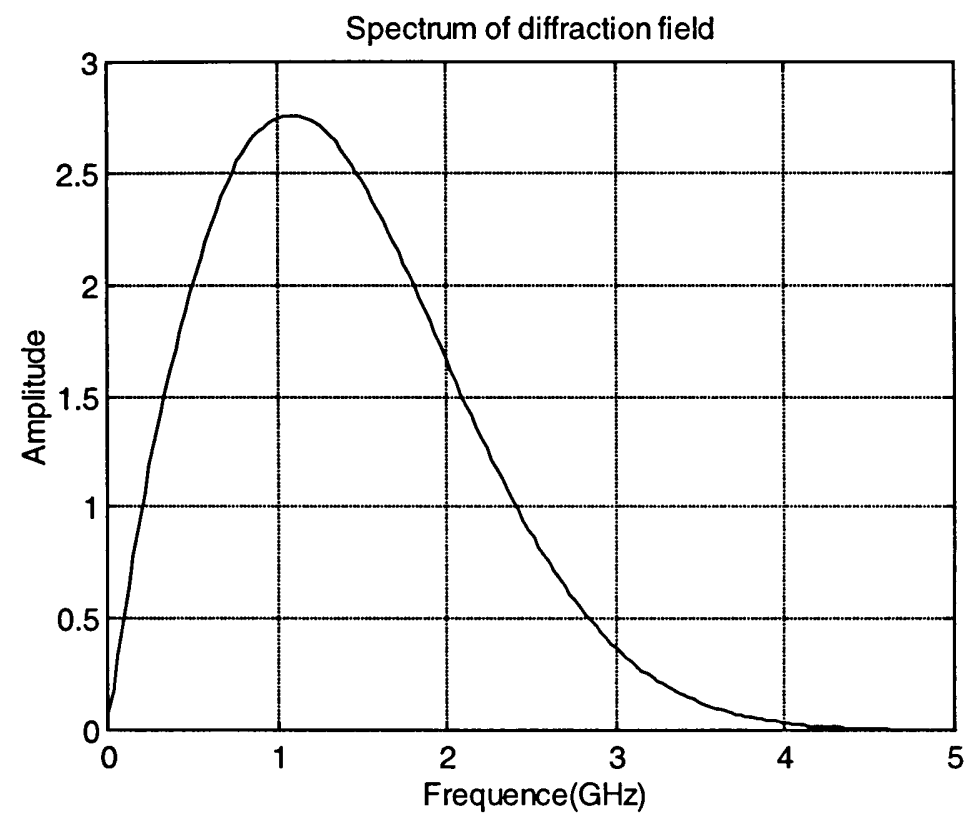

Figure 3.16 Spectrum of the diffraction field from $\theta$-polarized plane wave incident on PEC wedge for $\phi=35^{\circ}$

Apply equation (2.21) for each frequency to get the diffraction coefficients.

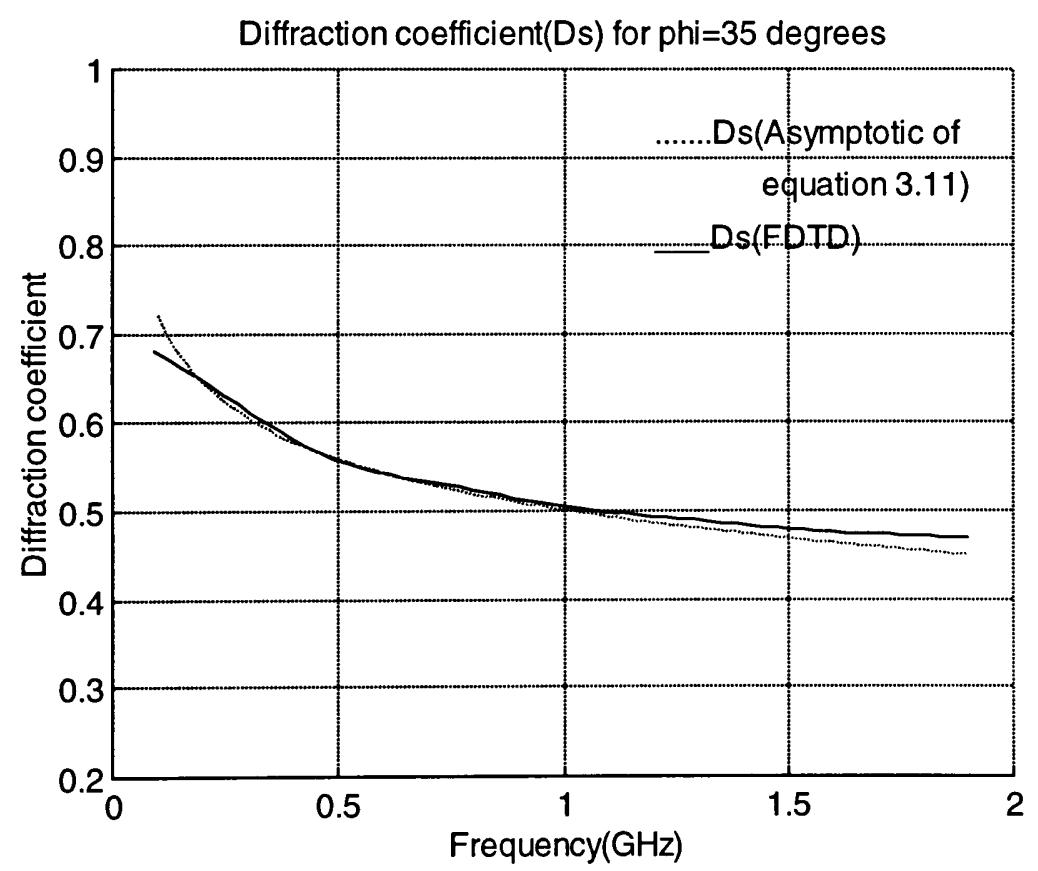

Figure 3.17 Comparison of soft diffraction coefficient $D_{s}$ from PEC wedge for $\phi=35^{\circ}$ by asymptotic and FDTD 
2) Hard Diffraction Coefficient $D_{h}$ for $\phi=35^{\circ}$

Set the incident modulated Gaussian pulse plane wave in $\phi$ polarization. The time domain waveform and spectrum will be same as the $\theta$-polarized pulse. Save scattered fields $E_{x}$ and $E_{y}$ at the observation point R for hard diffraction coefficient $D_{h}$.

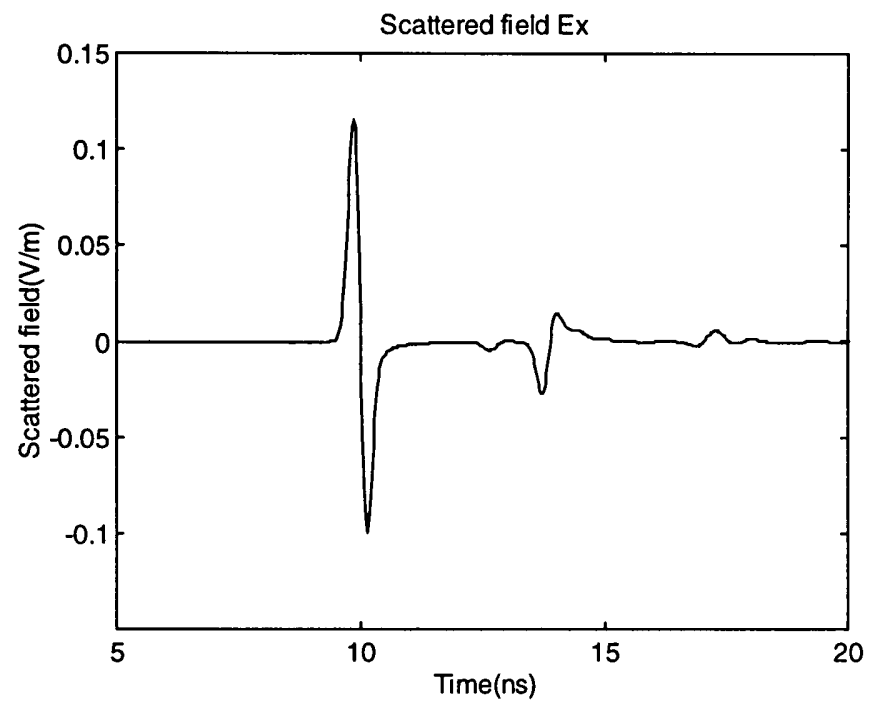

Figure 3.18 Scattered field Ex at receiver from $\phi$-polarized plane wave incident on PEC wedge for $\phi=35^{\circ}$

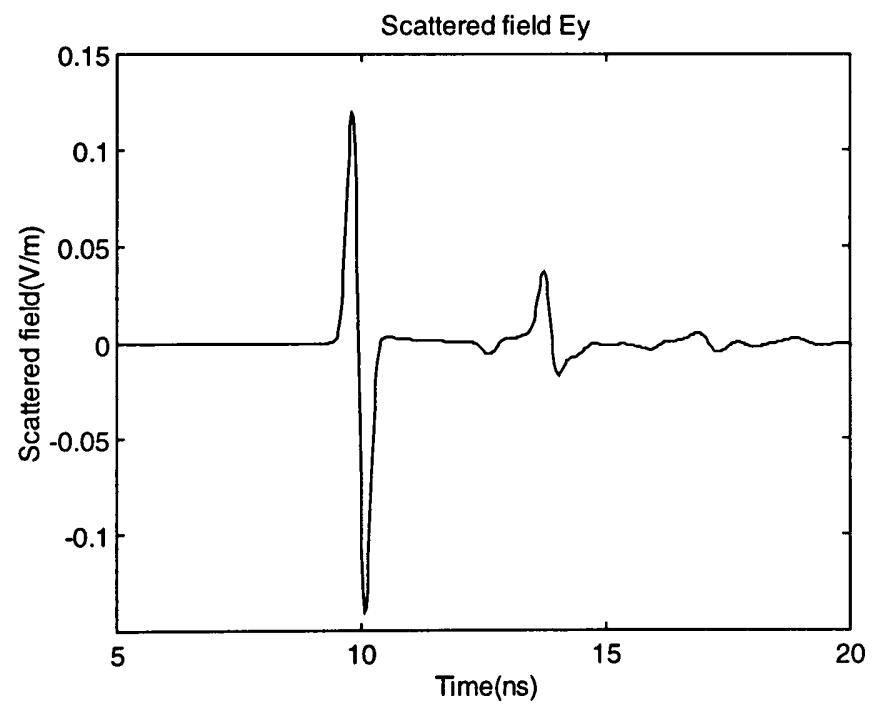

Figure 3.19 Scattered field Ey at receiver from $\phi$-polarized plane wave incident on PEC wedge for $\phi=35^{\circ}$ 
Use coordinate transformation

$$
E_{\phi}=-E_{x} \sin \phi+E_{y} \cos \phi
$$

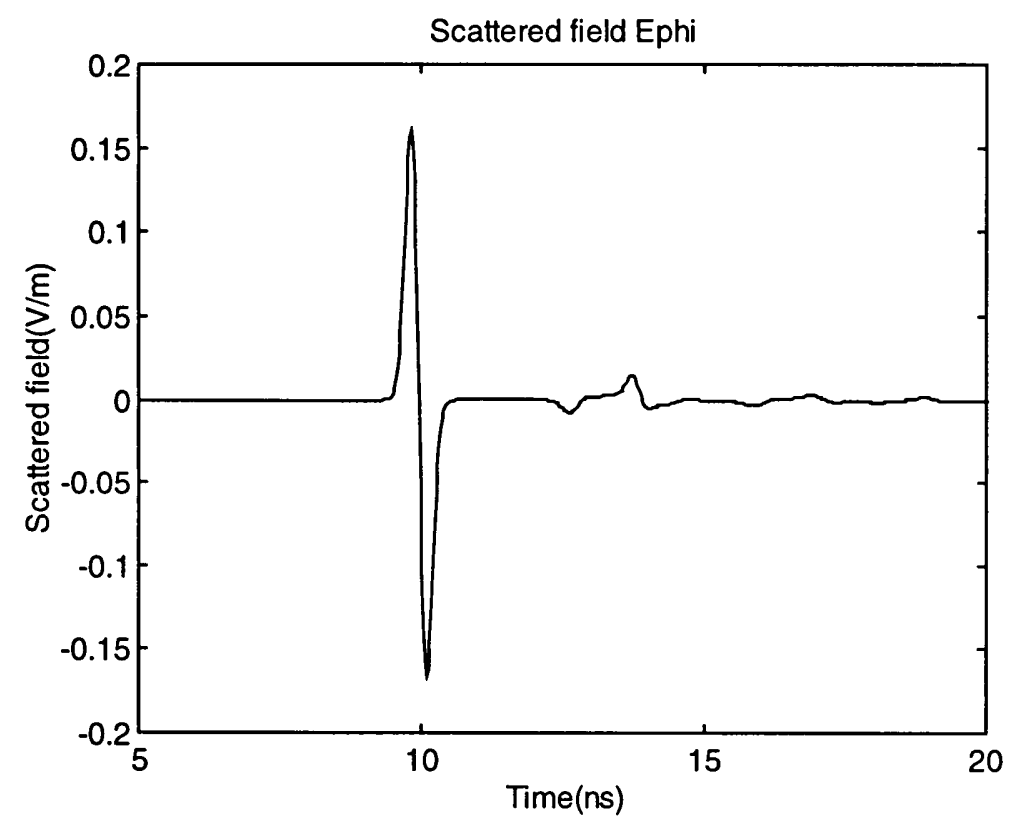

Figure 3.20 Scattered field at receiver from $\phi$-polarized plane wave incident on PEC wedge for $\phi=35^{\circ}$

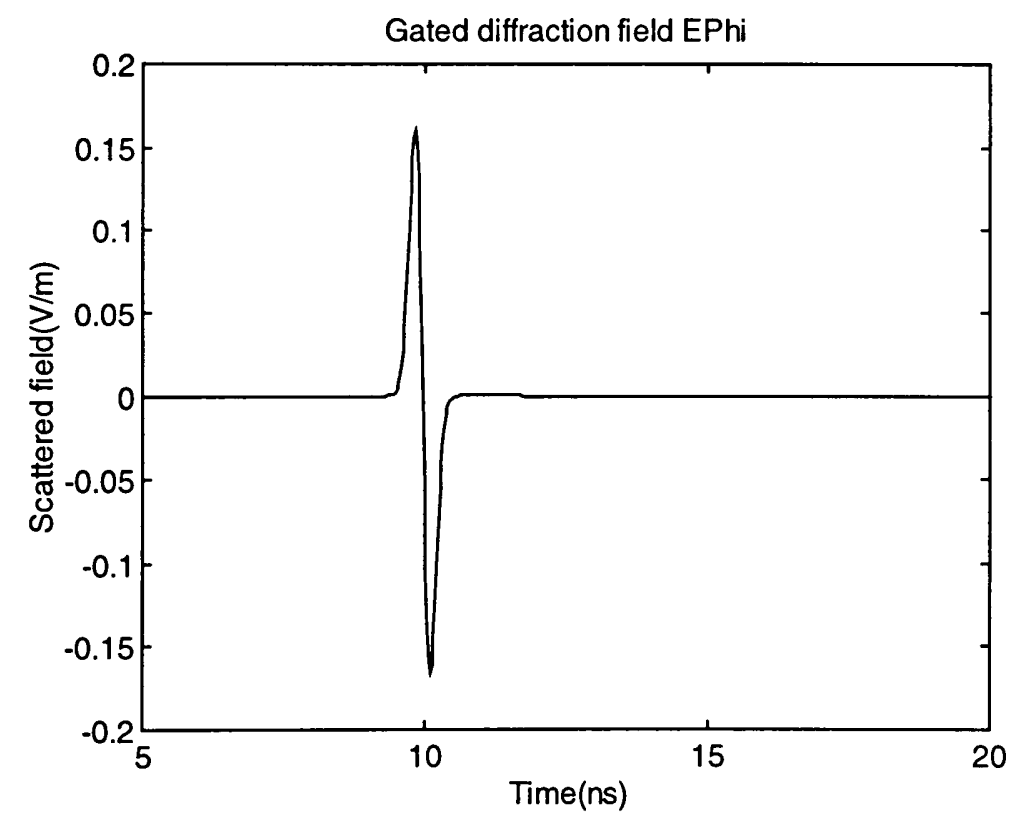

Figure 3.21 Extract the diffraction field from $\phi$-polarized plane wave incident on PEC wedge for $\phi=35^{\circ}$ using time-gating 


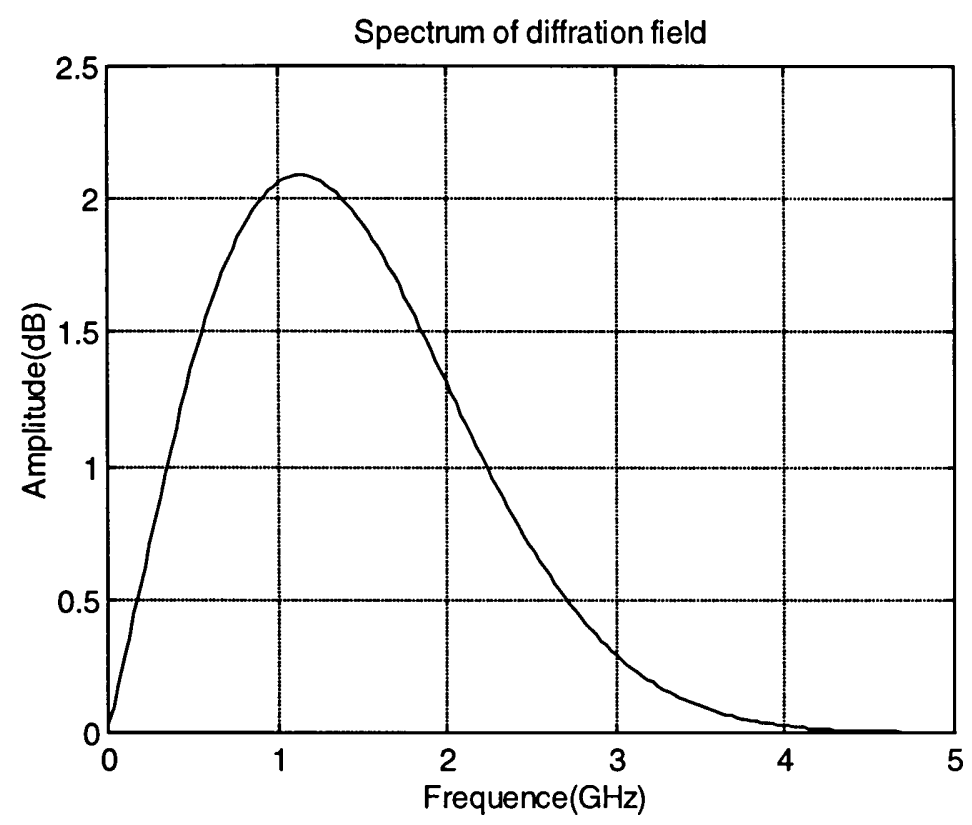

Figure 3.22 Spectrum of the diffraction field from $\phi$-polarized plane wave incident on PEC wedge for $\phi=35^{\circ}$

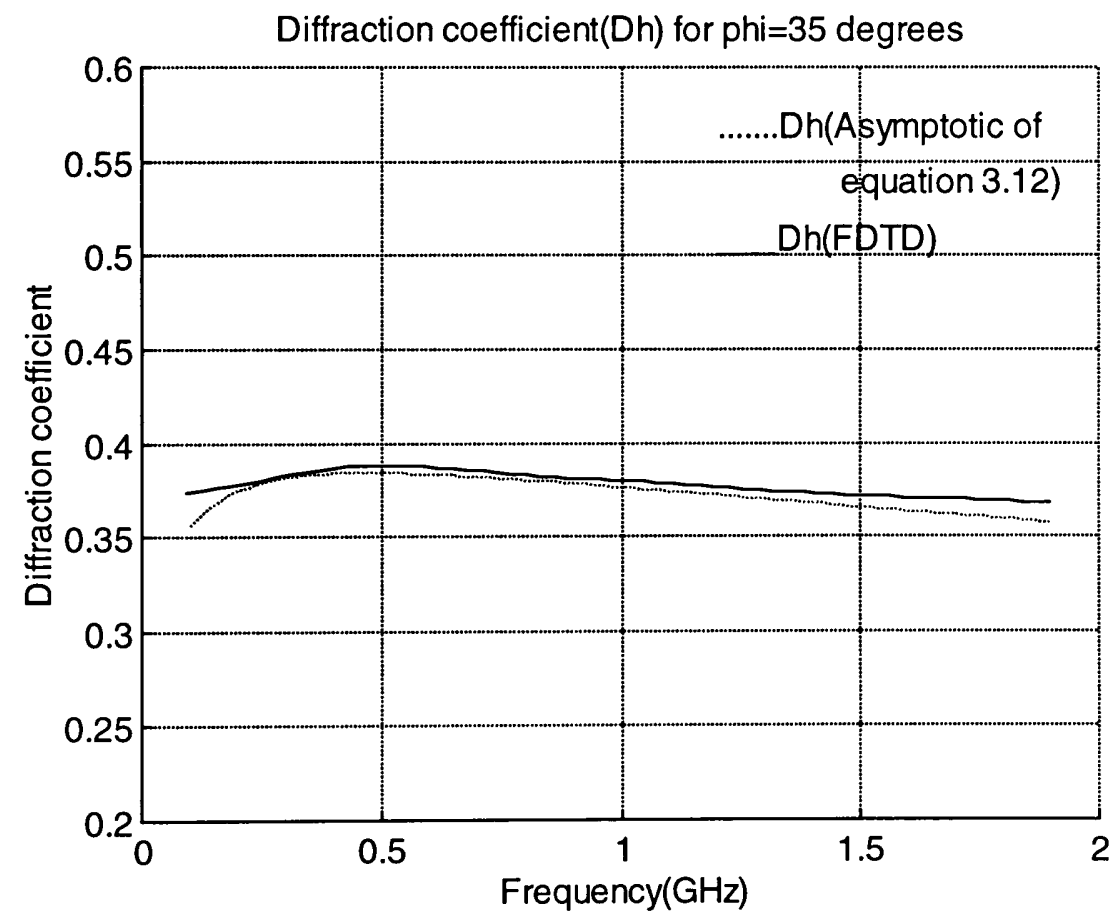

Figure 3.23 Comparison of hard diffraction coefficient $D_{h}$ from PEC wedge for $\phi=35^{\circ}$ by asymptotic and FDTD 
3.3.2.2 Diffraction Coefficients of PEC Wedge for $\phi^{\prime}=150^{\circ}, \beta^{\prime}=\mathbf{7 0}^{\circ}, \phi=40^{\circ}$

$X=149$
$Y=130$
$Z=300$
$X=99$
$y=40$
$Z=280$
$R(94,35,210)$

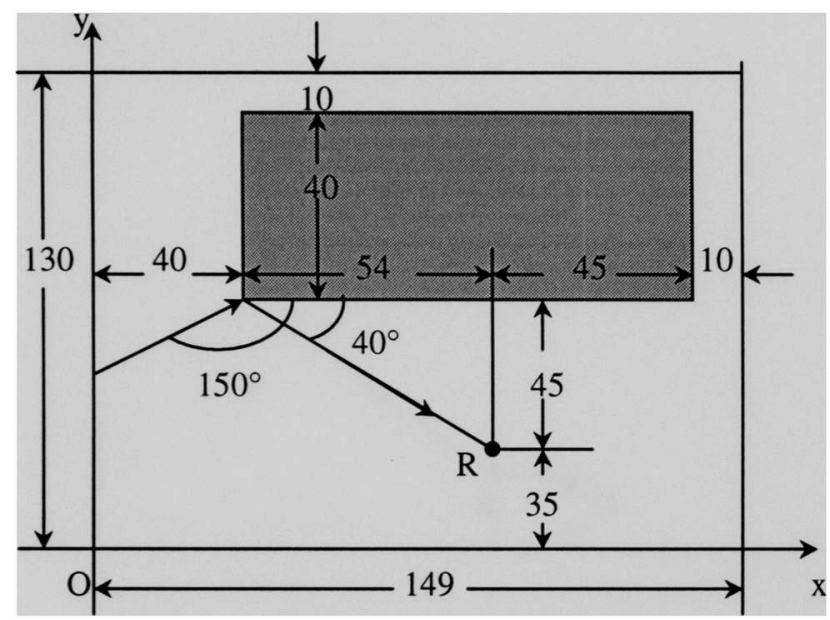

Figure 3.24 Geometry of structure and problem space to get the diffraction coefficient for $\phi^{\prime}=150^{\circ}, \beta^{\prime}=70^{\circ}$ and $\phi=40^{\circ}$ in $\mathrm{R}=1.06 \mathrm{~m}$

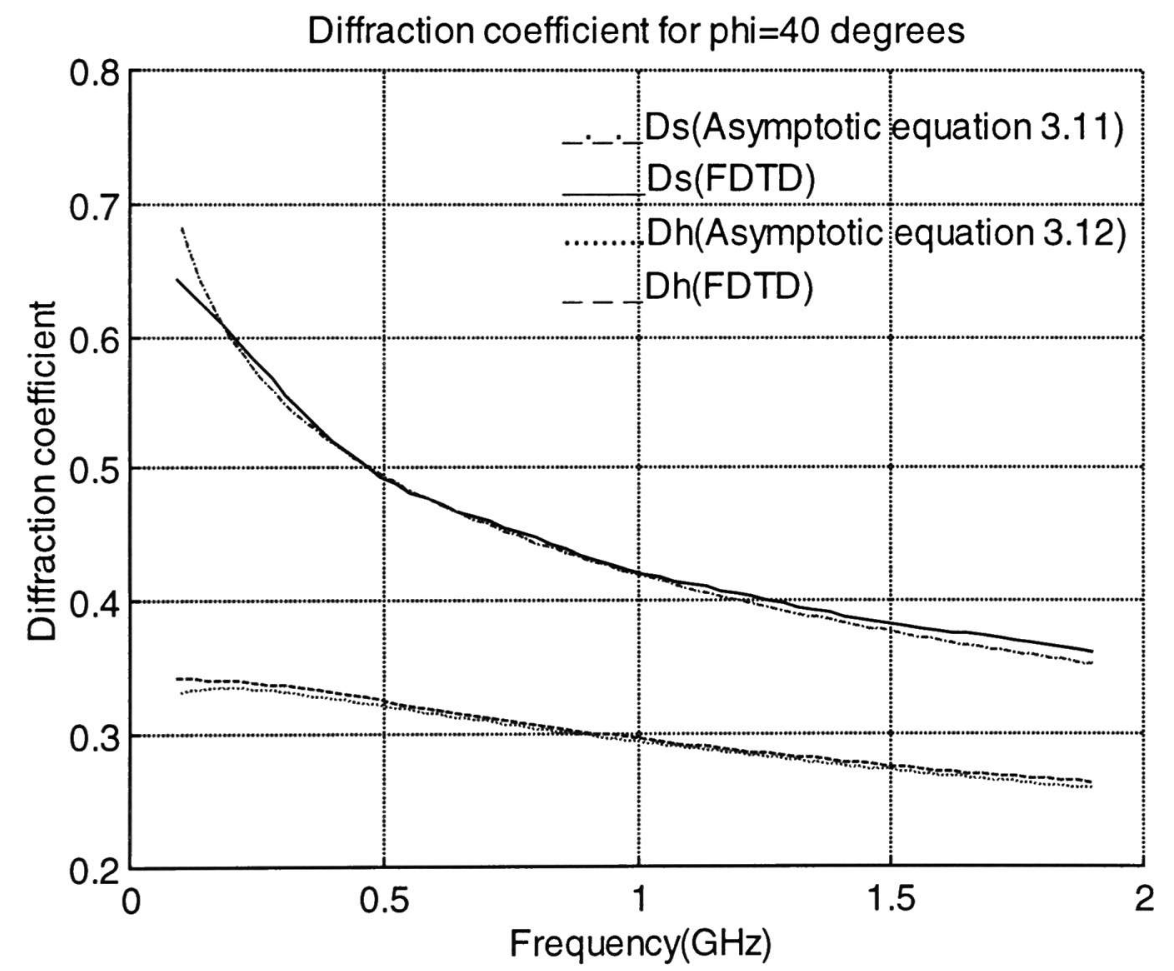

Figure 3.25 Comparison of soft and hard diffraction coefficients for PEC wedge at $1.06 \mathrm{~m}\left(\beta^{\prime}=70^{\circ}, \phi^{\prime}=150^{\circ}, \phi=40^{\circ}\right)$ by asymptotic and FDTD 
3.3.2.3 Diffraction Coefficients of PEC Wedge for $\phi^{\prime}=150^{\circ}, \beta^{\prime}=70^{\circ}, \phi=45^{\circ}$

$X=143$
$Y=138$
$Z=300$
$x=93$
$y=43$
$z=280$
$R(90,35,210)$

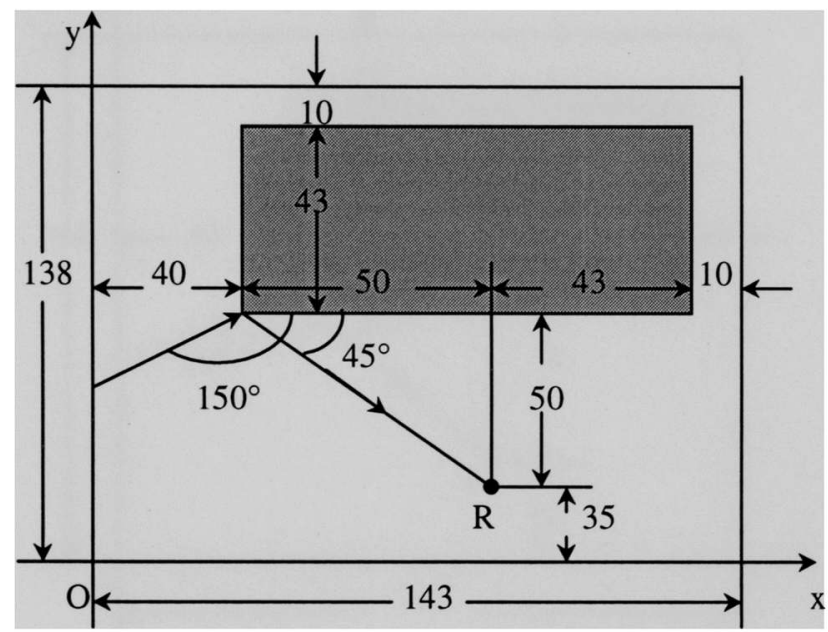

Figure 3.26 Geometry of structure and problem space to get the diffraction coefficient for $\phi^{\prime}=150^{\circ}, \beta^{\prime}=70^{\circ}$ and $\phi=45^{\circ}$ in $\mathrm{R}=1.06 \mathrm{~m}$

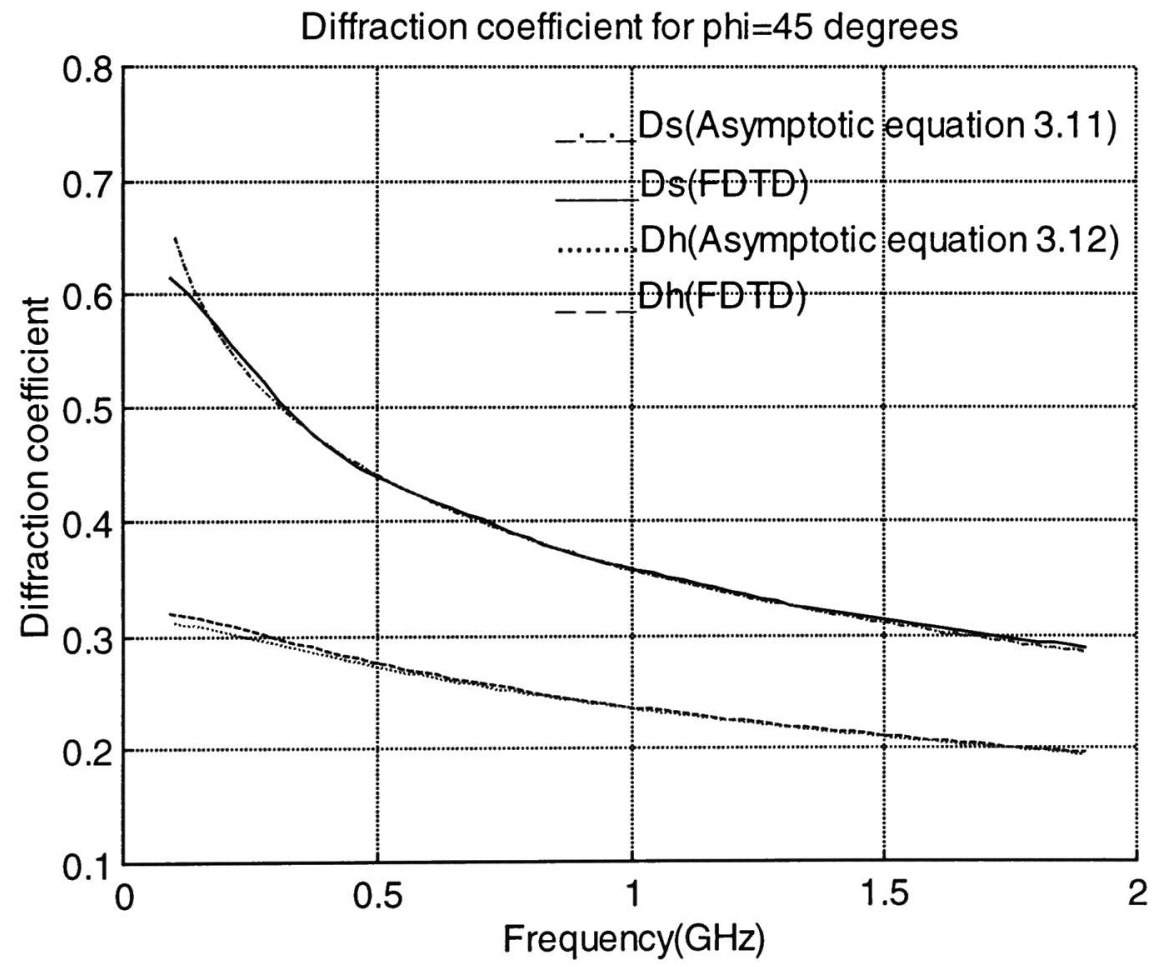

Figure 3.27 Comparison of soft and hard diffraction coefficients for PEC wedge at $1.06 \mathrm{~m}\left(\beta^{\prime}=70^{\circ}, \phi^{\prime}=150^{\circ}, \phi=45^{\circ}\right)$ by asymptotic and FDTD 
3.3.2.4 Diffraction Coefficients of PEC Wedge for $\phi^{\prime}=150^{\circ}, \beta^{\prime}=70^{\circ}, \phi=50^{\circ}$

$$
\begin{aligned}
& X=138 \\
& Y=144 \\
& Z=300 \\
& X=88 \\
& y=45 \\
& z=280 \\
& R(85,35,210)
\end{aligned}
$$

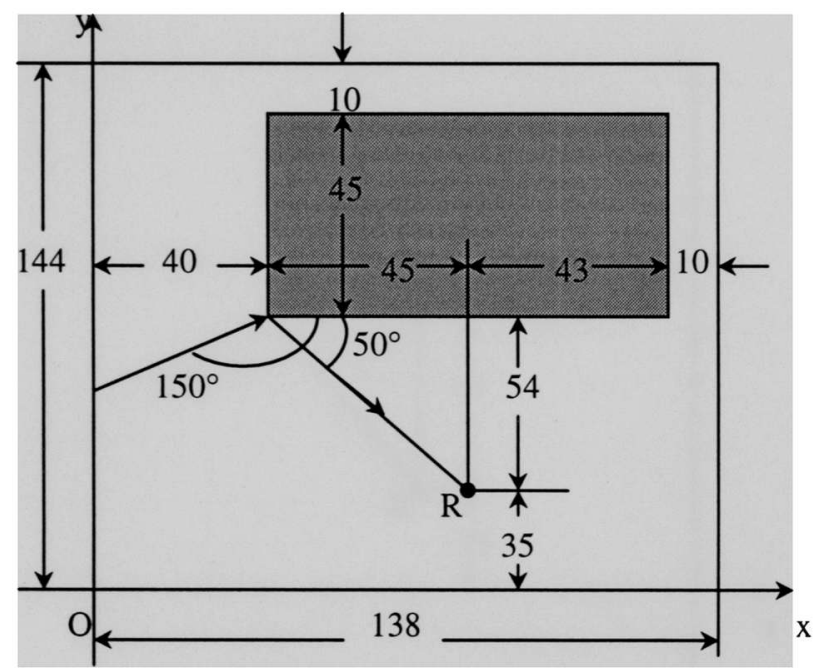

Figure 3.28 Geometry of structure and problem space to get the diffraction coefficient for $\phi^{\prime}=150^{\circ}, \beta^{\prime}=70^{\circ}$ and $\phi=50^{\circ}$ in $\mathrm{R}=1.06 \mathrm{~m}$

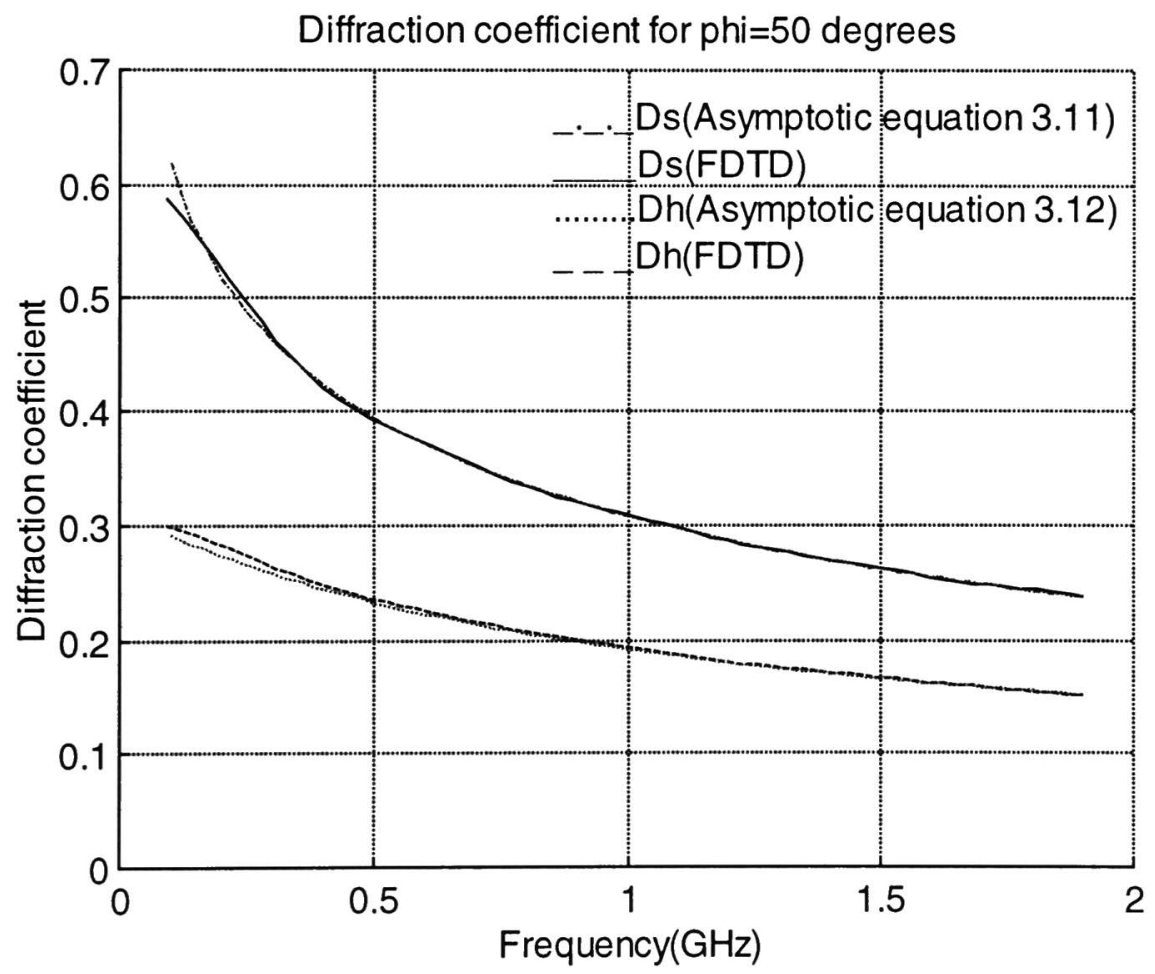

Figure 3.29 Comparison of soft and hard diffraction coefficients for PEC wedge at $1.06 \mathrm{~m}\left(\beta^{\prime}=70^{\circ}, \phi^{\prime}=150^{\circ}, \phi=50^{\circ}\right)$ by asymptotic and FDTD 
3.3.2.5 Diffraction Coefficients of PEC Wedge for $\phi^{\prime}=150^{\circ}, \beta^{\prime}=70^{\circ}, \phi=60^{\circ}$

$X=138$
$Y=154$
$Z=300$
$x=78$
$y=48$
$z=280$
$R(85,35,210)$

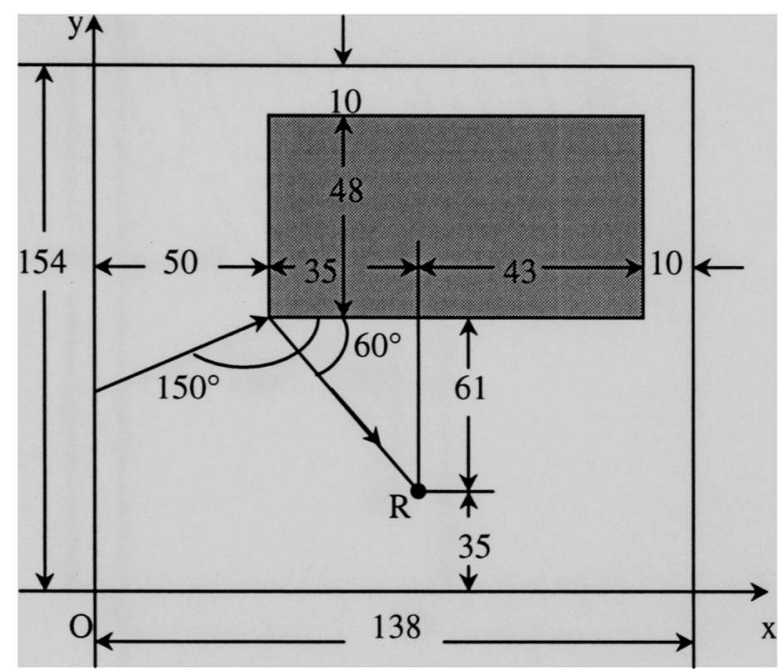

Figure 3.30 Geometry of structure and problem space to get the diffraction coefficient for $\phi^{\prime}=150^{\circ}, \beta^{\prime}=70^{\circ}$ and $\phi=60^{\circ}$ in $\mathrm{R}=1.06 \mathrm{~m}$

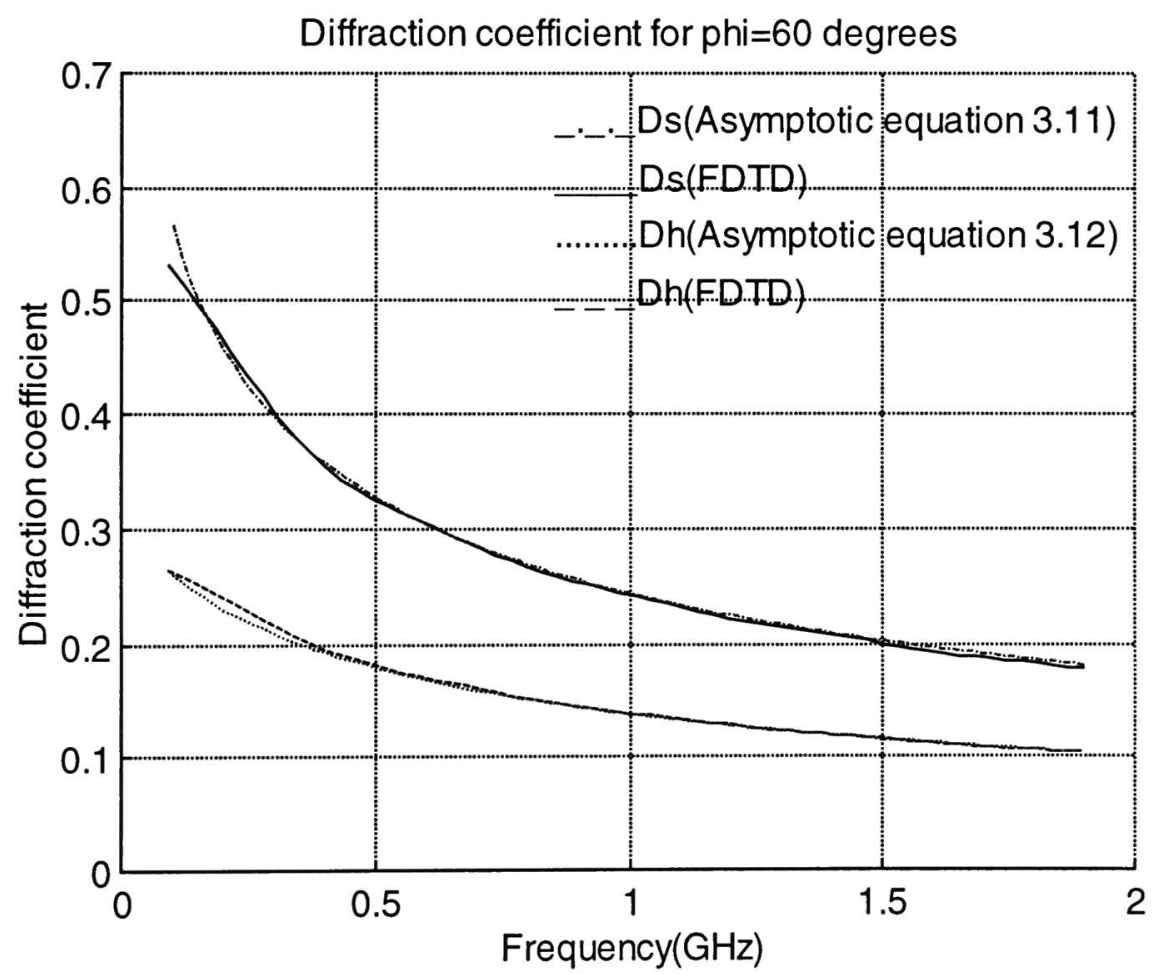

Figure 3.31 Comparison of soft and hard diffraction coefficients for PEC wedge at $1.06 \mathrm{~m}\left(\beta^{\prime}=70^{\circ}, \phi^{\prime}=150^{\circ}, \phi=60^{\circ}\right)$ by asymptotic and FDTD 
3.3.2.6 Diffraction Coefficients of PEC Wedge for $\phi^{\prime}=150^{\circ}, \beta^{\prime}=7^{\circ}, \phi=70^{\circ}$

$X=136$
$Y=163$
$Z=300$
$X=76$
$y=52$
$Z=280$
$R(74,35,210)$

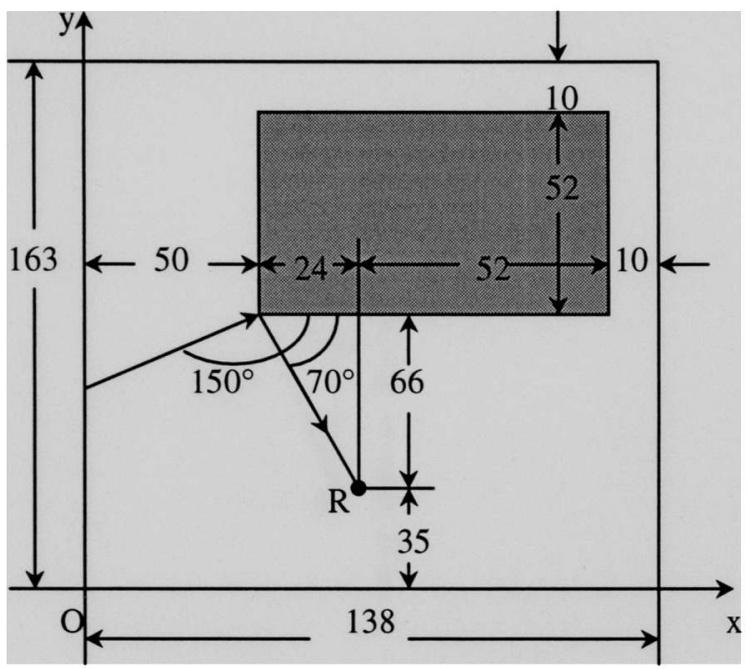

Figure 3.32 Geometry of structure and problem space to get the diffraction coefficient for $\phi^{\prime}=150^{\circ}, \beta^{\prime}=70^{\circ}$ and $\phi=70^{\circ}$ in $\mathrm{R}=1.06 \mathrm{~m}$

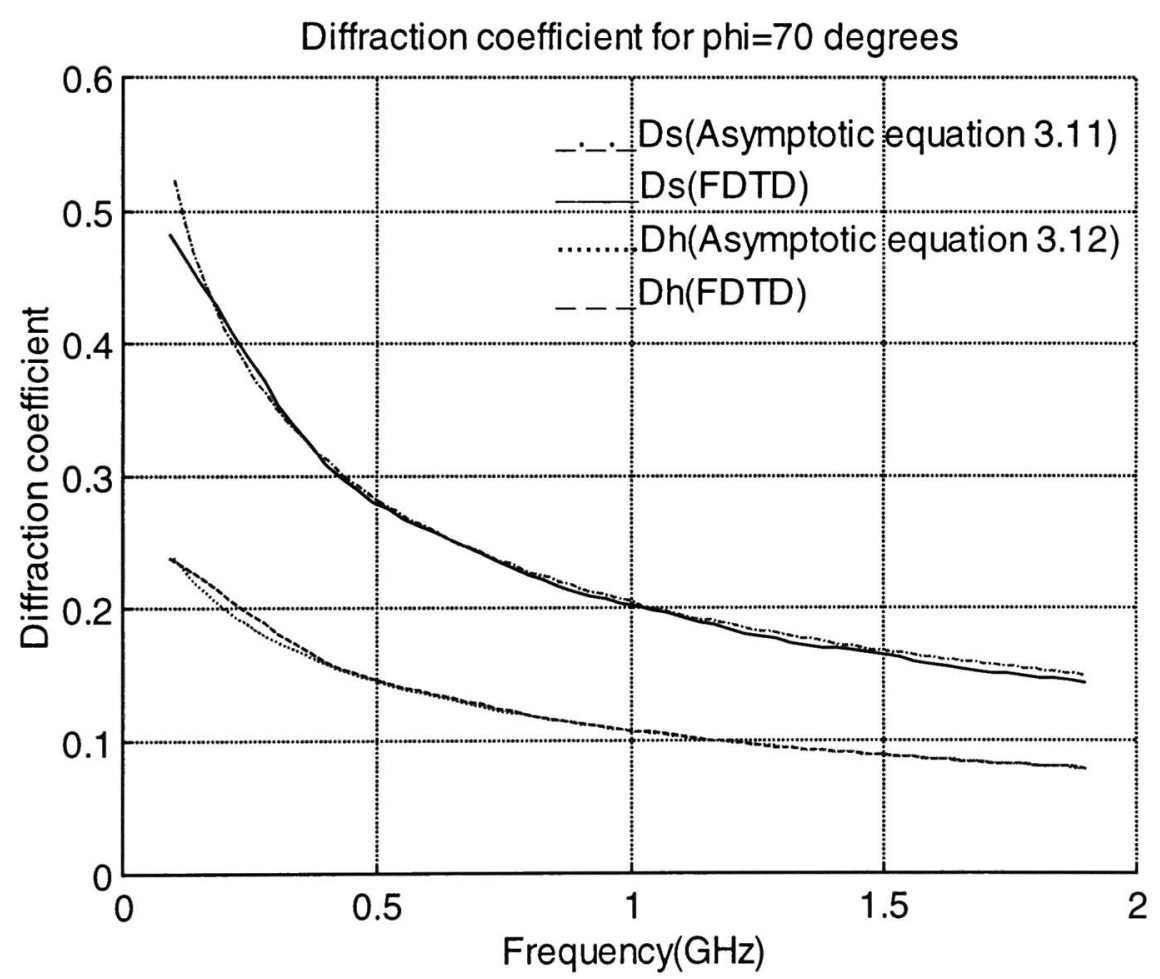

Figure 3.33 Comparison of soft and hard diffraction coefficients for PEC wedge at $1.06 \mathrm{~m}\left(\beta^{\prime}=70^{\circ}, \phi^{\prime}=150^{\circ}, \phi=70^{\circ}\right)$ by asymptotic and FDTD 
3.3.2.7 Diffraction Coefficients of PEC Wedge for $\phi^{\prime}=150^{\circ}, \beta^{\prime}=\mathbf{7 0}^{\circ}, \phi=80^{\circ}$

$$
\begin{aligned}
& X=134 \\
& Y=170 \\
& Z=300 \\
& X=64 \\
& y=56 \\
& Z=280 \\
& R(72,35,210)
\end{aligned}
$$

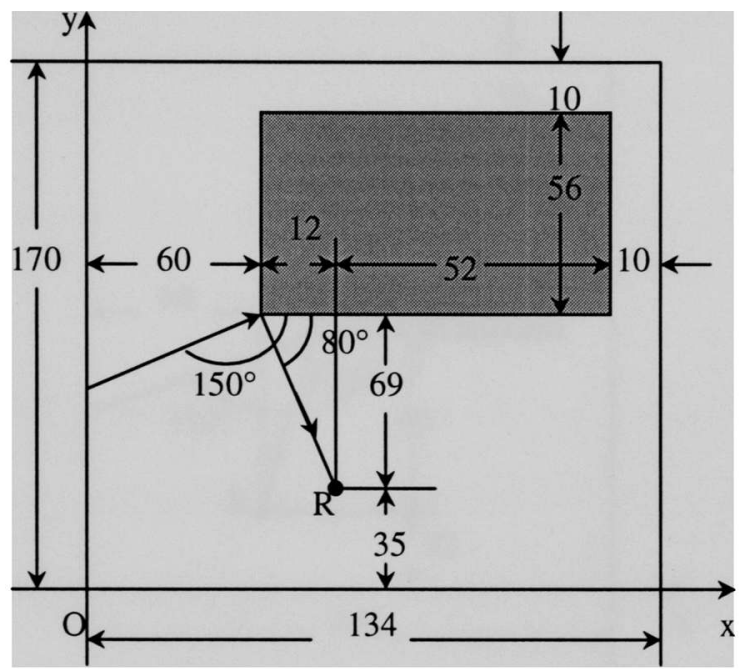

Figure 3.34 Geometry of structure and problem space to get the diffraction coefficient for $\phi^{\prime}=150^{\circ}, \beta^{\prime}=70^{\circ}$ and $\phi=80^{\circ}$ in $\mathrm{R}=1.06 \mathrm{~m}$

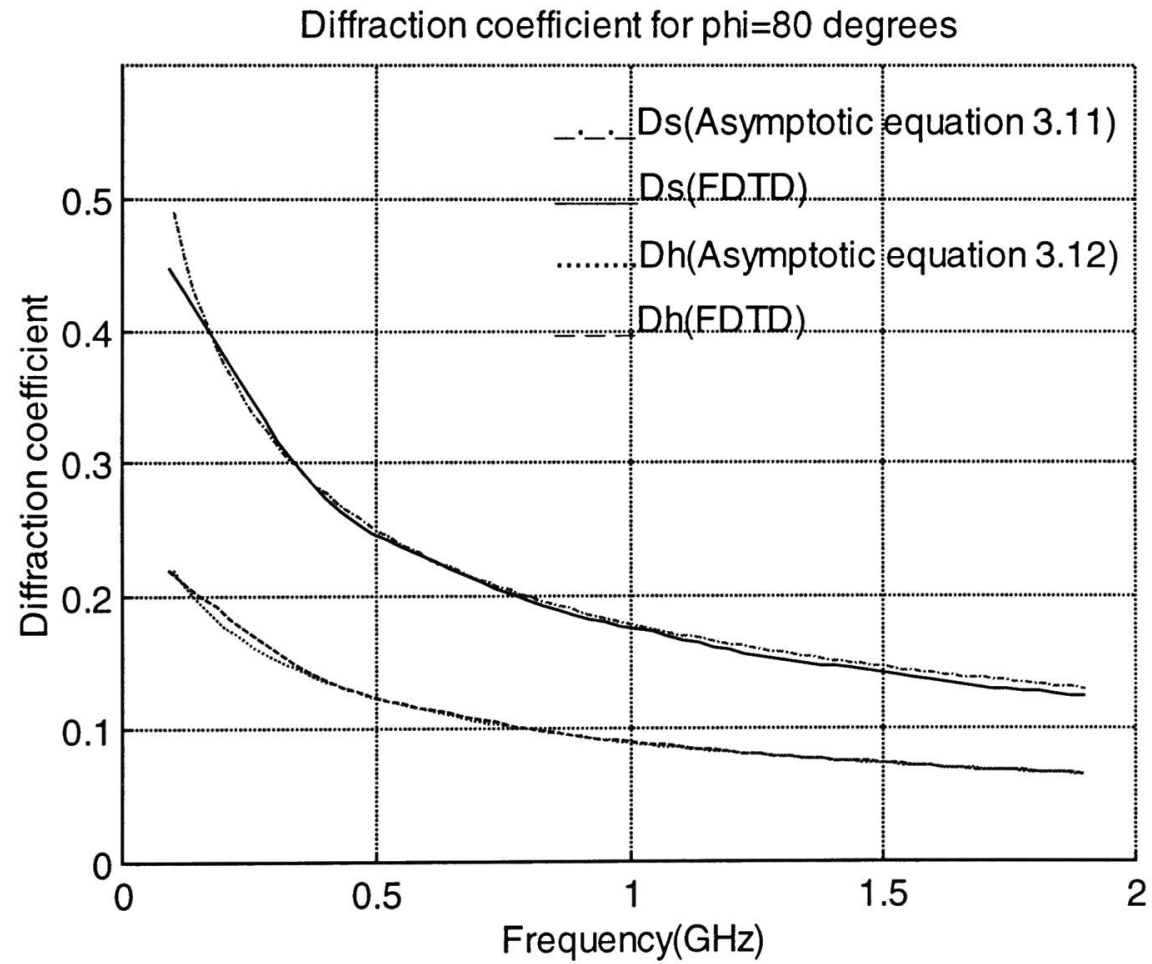

Figure 3.35 Comparison of soft and hard diffraction coefficients for PEC wedge at $1.06 \mathrm{~m}\left(\beta^{\prime}=70^{\circ}, \phi^{\prime}=150^{\circ}, \phi=80^{\circ}\right)$ by asymptotic and FDTD 
3.3.2.8 Diffraction Coefficients of PEC Wedge for $\phi^{\prime}=150^{\circ}, \beta^{\prime}=7^{\circ}, \phi=100^{\circ}$

$$
\begin{aligned}
& X=146 \\
& Y=160 \\
& Z=300 \\
& x=64 \\
& y=58 \\
& z=280 \\
& R(60,23,210)
\end{aligned}
$$

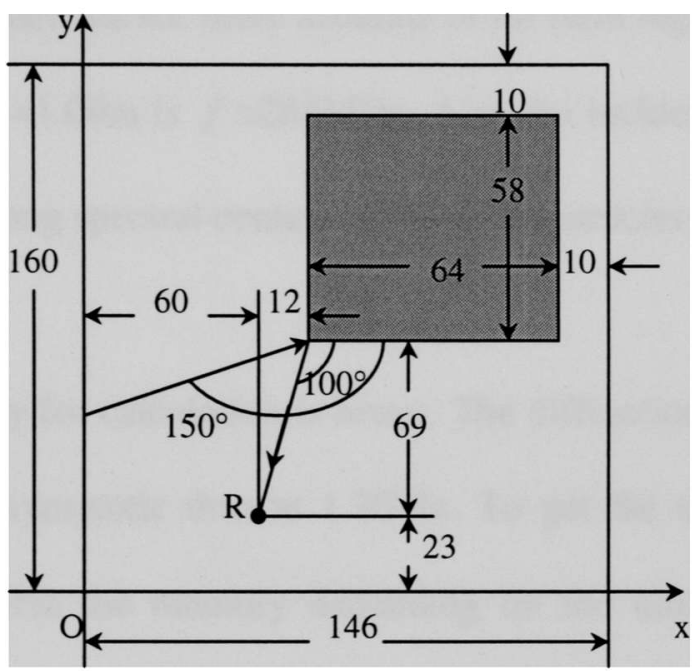

Figure 3.36 Geometry of structure and problem space to get the diffraction coefficient for $\phi^{\prime}=150^{\circ}, \beta^{\prime}=70^{\circ}$ and $\phi=100^{\circ}$ in $\mathrm{R}=1.06 \mathrm{~m}$

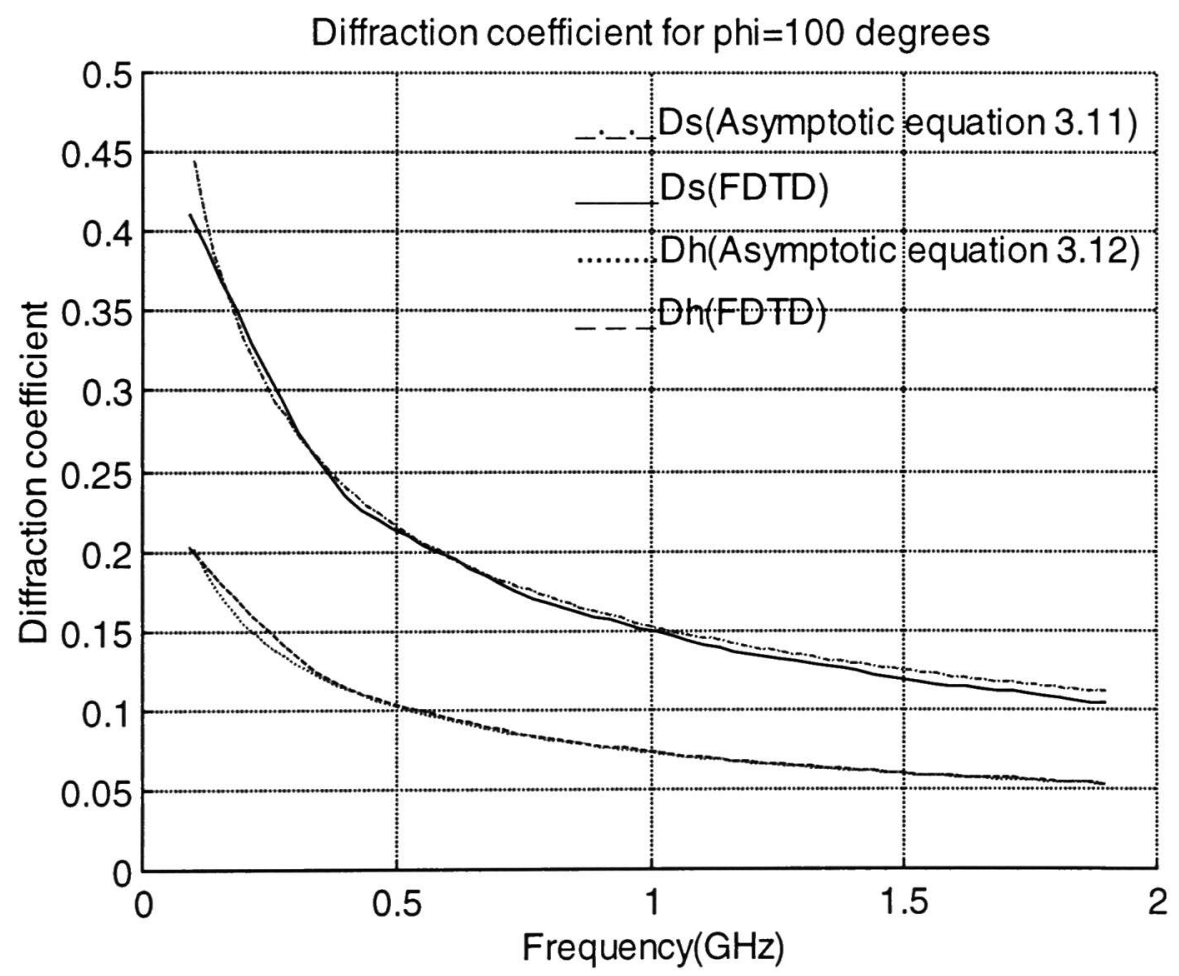

Figure 3.37 Comparison of soft and hard diffraction coefficients for PEC wedge at $1.06 \mathrm{~m}\left(\beta^{\prime}=70^{\circ}, \phi^{\prime}=150^{\circ}, \phi=100^{\circ}\right)$ by asymptotic and FDTD 
Some mismatches are at the low and high frequencies.

- The asymptotic diffraction coefficients are more accurate in far field region. If $d>\lambda$ is the far zone condition, for $d=1.06 \mathrm{~m}$ is $f>283 \mathrm{MHz}$. Also the incident modulated Gaussian pulse doesn't have strong spectral content at lower frequencies to illuminate the wedge.

- At high frequencies the accuracy for calculation is lower. The diffraction coefficients at $850 \mathrm{MHz}$ are closer to the asymptotic than at $1.7 \mathrm{GHz}$. To get the same level of answer at $1.7 \mathrm{GHz}$ as at $850 \mathrm{MHz}$ the memory depending on the number of cells should be doubled which could be not practical. Although the diffraction coefficients at $1.7 \mathrm{GHz}$ have lower accuracy the data are still close to analytical results and valid in FDTD frequency response.

Pick up the diffraction coefficients at frequencies most close to $850 \mathrm{MHz}$ and $1.7 \mathrm{GHz}$ while the frequency resolution $\Delta f$ is defined by time step $\Delta t$ and the total number of time steps $T$ as

$$
\Delta f=\frac{1}{T \cdot \Delta t}
$$

Connect the soft diffraction coefficients $D_{s}$ and hard diffraction coefficients $D_{h}$ respectively and compare with asymptotic for PEC wedge. 


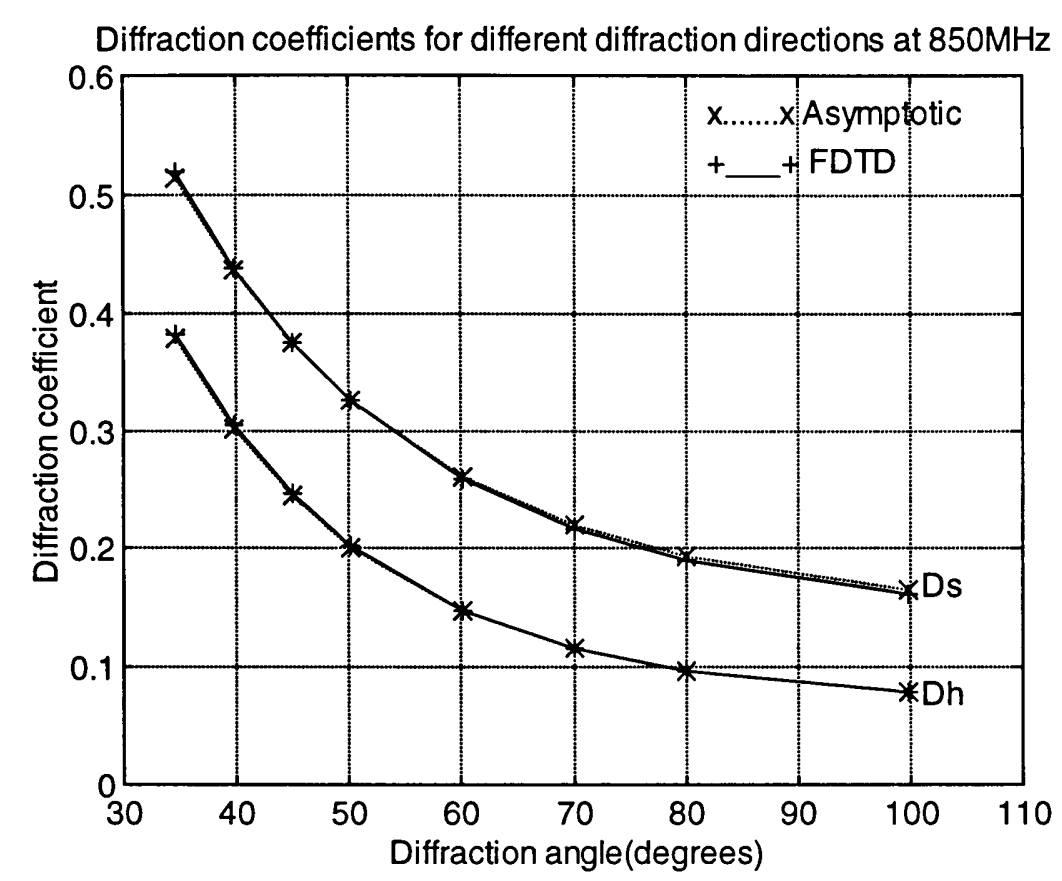

Figure 3.38 Comparison of soft and hard diffraction coefficients at $850 \mathrm{MHz}$ to different directions for PEC wedge at $1.06 \mathrm{~m}\left(\beta^{\prime}=70^{\circ}, \phi^{\prime}=150^{\circ}\right)$ by asymptotic and FDTD

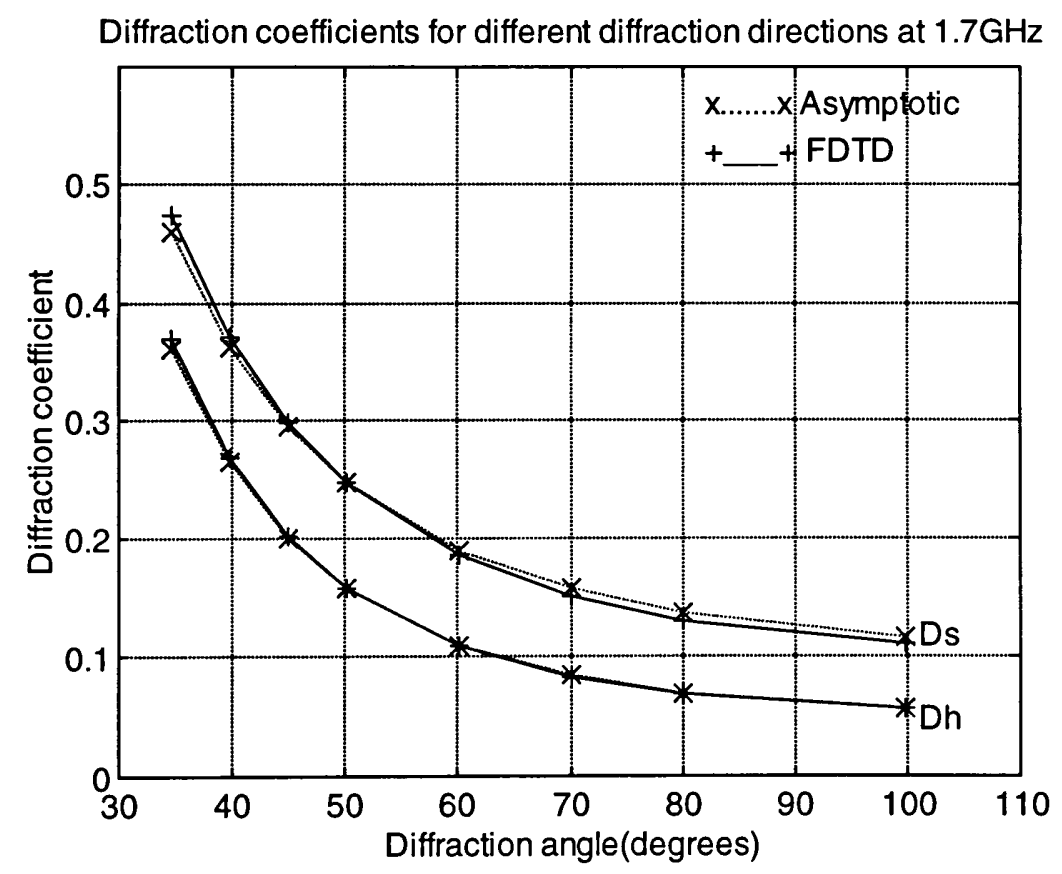

Figure 3.39 Comparison of soft and hard diffraction coefficients at $1.7 \mathrm{GHz}$ to different directions for PEC wedge at $1.06 \mathrm{~m}\left(\beta^{\prime}=70^{\circ}, \phi^{\prime}=150^{\circ}\right)$ by asymptotic and FDTD 


\subsection{3-Dimensional Diffraction Coefficient of Dielectric Wedges}

Based on the agreement of diffraction coefficients for PEC wedge obtained using FDTD method and the asymptotic equations, we can extend the wedges with practical dielectric materials for diffraction coefficients that are not available analytically.

Diffraction is not scattering propagation inside the scatterer, the cell size is not concerned to be smaller than in the free space for dielectric structure although the wavelength of dielectric is shorter. And for above geometry of PEC wedges it takes much time for the fields caused by internal propagation of the dielectric object to the observation point. Use the same cell size and geometry as the PEC structures and change the material properties $\left(\varepsilon_{r}, \sigma\right)$ to building components which depends on the compositions

1. $(100,100)$

2. $(12,0.1)$

3. $(3,0.01)$

$\varepsilon=\varepsilon_{0} \varepsilon_{r}$ is the permittivity of the medium with $\varepsilon_{0}=8.85 \times 10^{-12} \mathrm{~F} / \mathrm{m} . \quad \sigma$ is the conductivity of the medium.

3.4.1 Diffraction Coefficients of Dielectric Wedge for $\phi^{\prime}=150^{\circ}, \beta^{\prime}=7^{\circ}, \phi=35^{\circ}$

3.4.1.1 Diffraction Coefficients for Wedge $\left(\varepsilon_{r}, \sigma=100,100\right)$ in $\phi=35^{\circ}$

1) Soft Diffraction Coefficients $D_{s}$ for Wedge $\left(\varepsilon_{r}, \sigma=100,100\right)$ in $\phi=35^{\circ}$ 


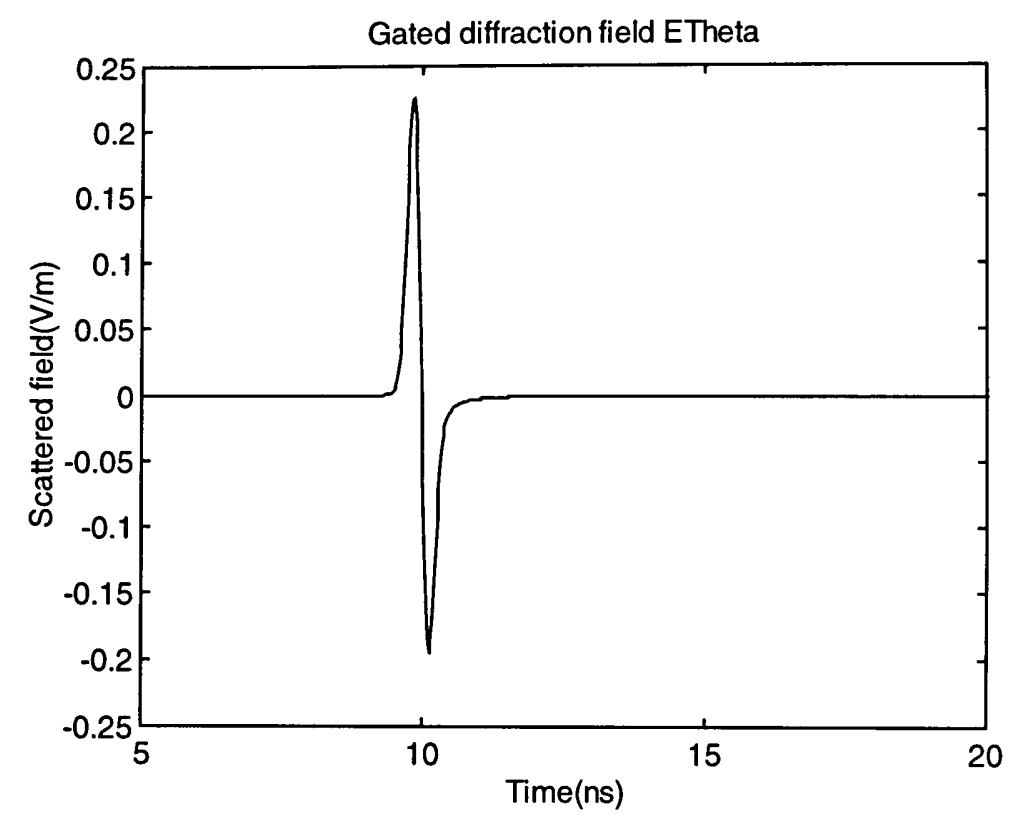

Figure 3.40 Diffraction field from $\theta$-polarized plane wave incident on wedge $\left(\varepsilon_{r}, \sigma=100,100\right)$ for $\phi=35^{\circ}$ using time-gating

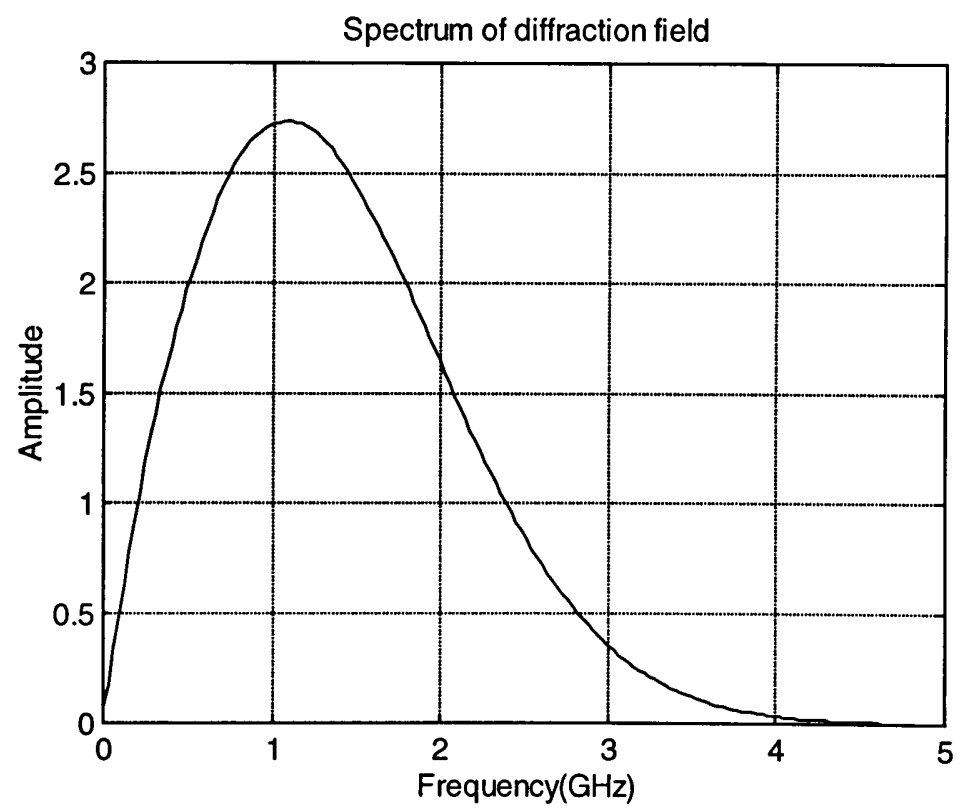

Figure 3.41 Spectrum of the diffraction field from $\theta$-polarized plane wave incident on wedge $\left(\varepsilon_{r}, \sigma=100,100\right)$ for $\phi=35^{\circ}$ 
Diffraction Coefficient(Ds) for phi=35 degrees (permittivity=100, conductivity=100)

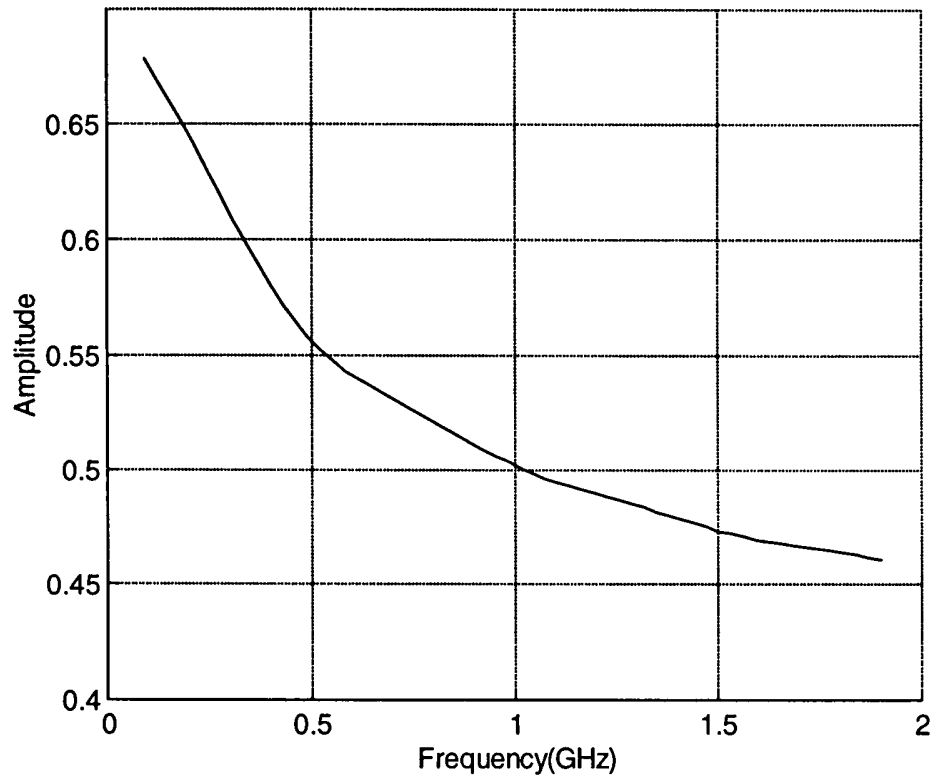

Figure 3.42 Soft diffraction coefficient of wedge $\left(\varepsilon_{r}, \sigma=100,100\right)$ for $\phi=35^{\circ}$ using FDTD

2) Hard Diffraction Coefficients $D_{h}$ for Wedge $\left(\varepsilon_{r}, \sigma=100,100\right)$ in $\phi=35^{\circ}$

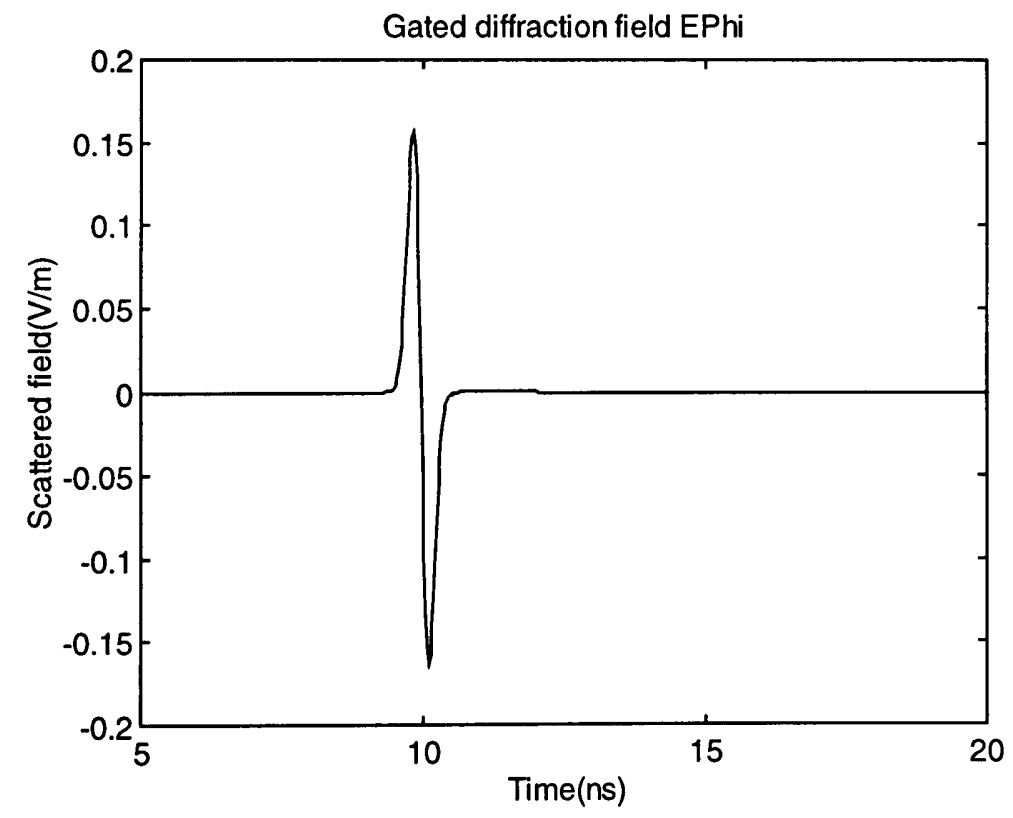

Figure 3.43 Diffraction field from $\phi$-polarized plane wave incident on wedge $\left(\varepsilon_{r}, \sigma=100,100\right)$ for $\phi=35^{\circ}$ using time-gating 


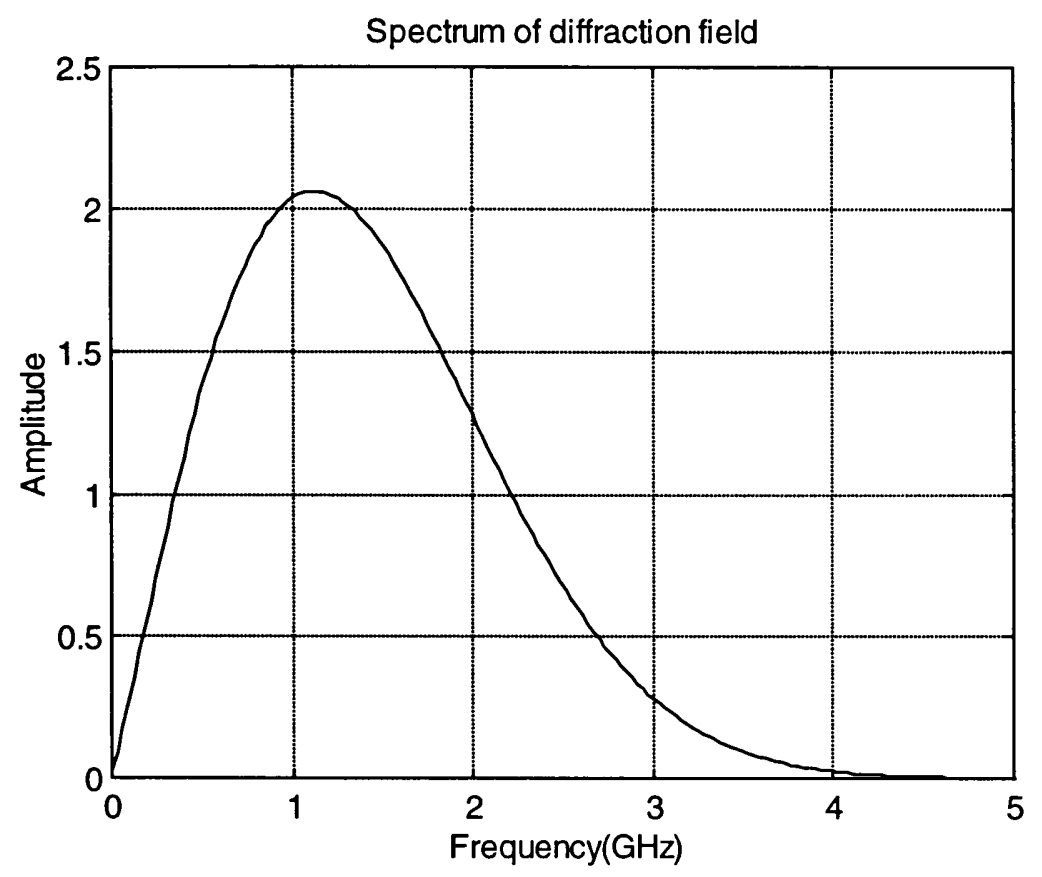

Figure 3.44 Spectrum of the diffraction field from $\phi$-polarized plane wave incident on wedge $\left(\varepsilon_{r}, \sigma=100,100\right)$ for $\phi=35^{\circ}$

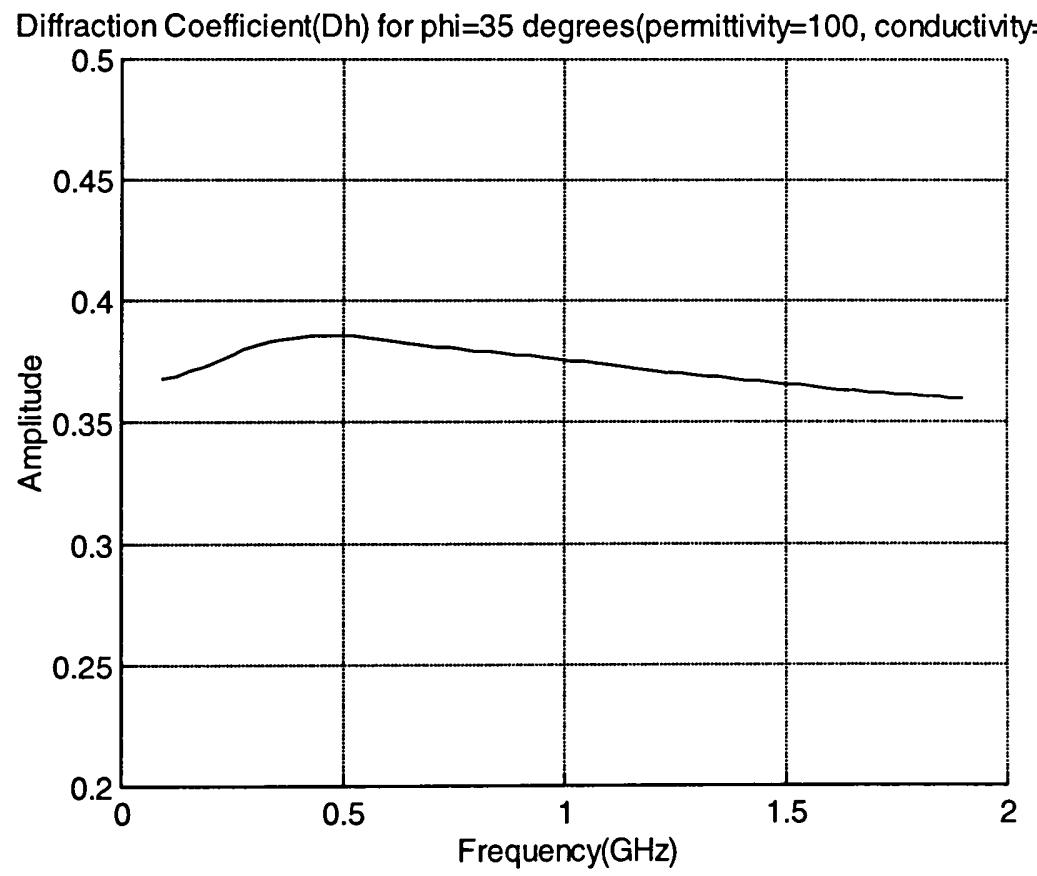

Figure 3.45 Hard diffraction coefficient of wedge $\left(\varepsilon_{r}, \sigma=100,100\right)$ for $\phi=35^{\circ}$ using FDTD 
3.4.1.3 Diffraction Coefficients for Wedge $\left(\varepsilon_{r}, \sigma=12,0.1\right)$ in $\phi=35^{\circ}$

1) Soft Diffraction Coefficients $D_{s}$ for Wedge $\left(\varepsilon_{r}, \sigma=12,0.1\right)$ in $\phi=35^{\circ}$

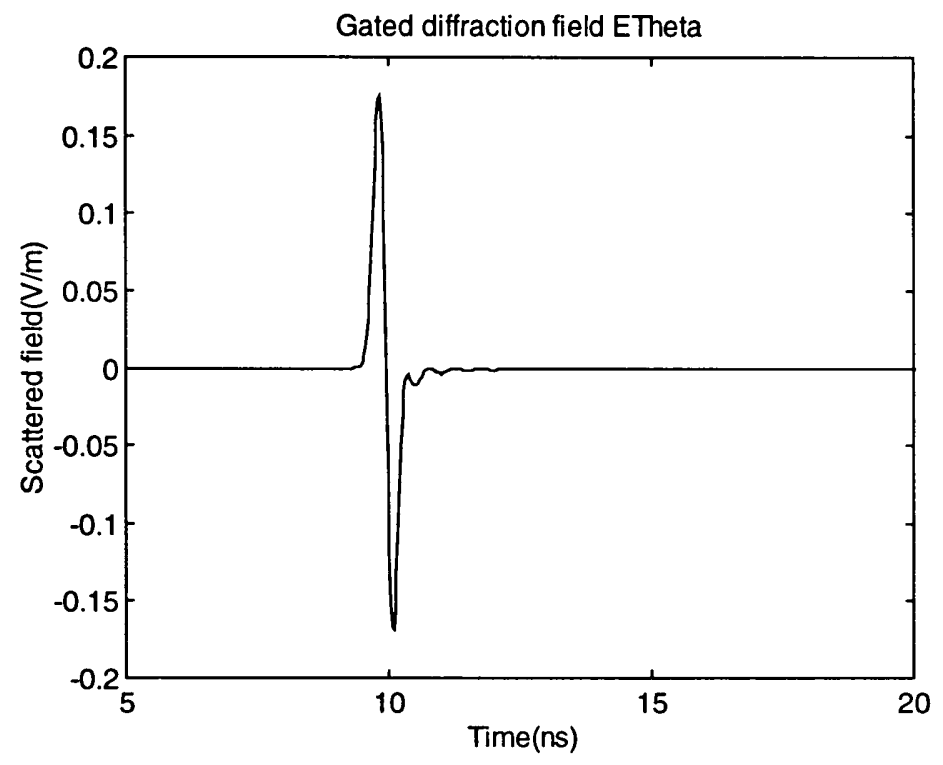

Figure 3.46 Diffraction field from $\theta$-polarized plane wave incident on wedge $\left(\varepsilon_{r}, \sigma=12,0.1\right)$ for $\phi=35^{\circ}$ using time-gating

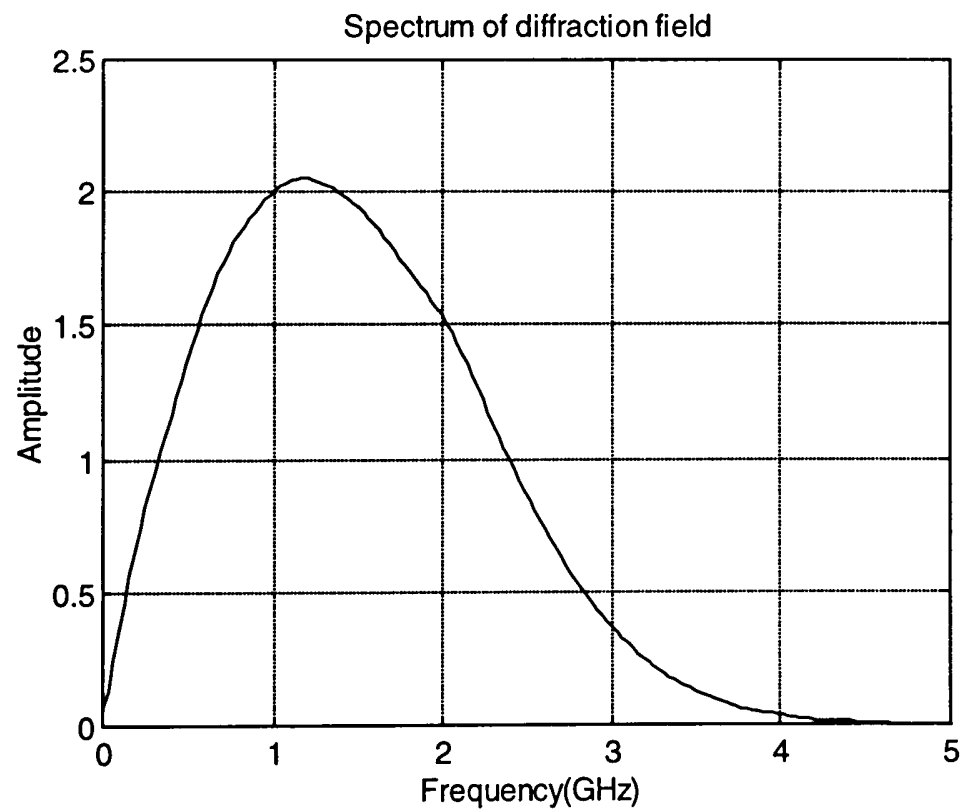

Figure 3.47 Spectrum of the diffraction field from $\theta$-polarized plane wave incident on wedge $\left(\varepsilon_{r}, \sigma=12,0.1\right)$ for $\phi=35^{\circ}$ 
Diffraction Coefficient(Ds) for phi=35 degrees(permittivity=12, conductivity=0.1)

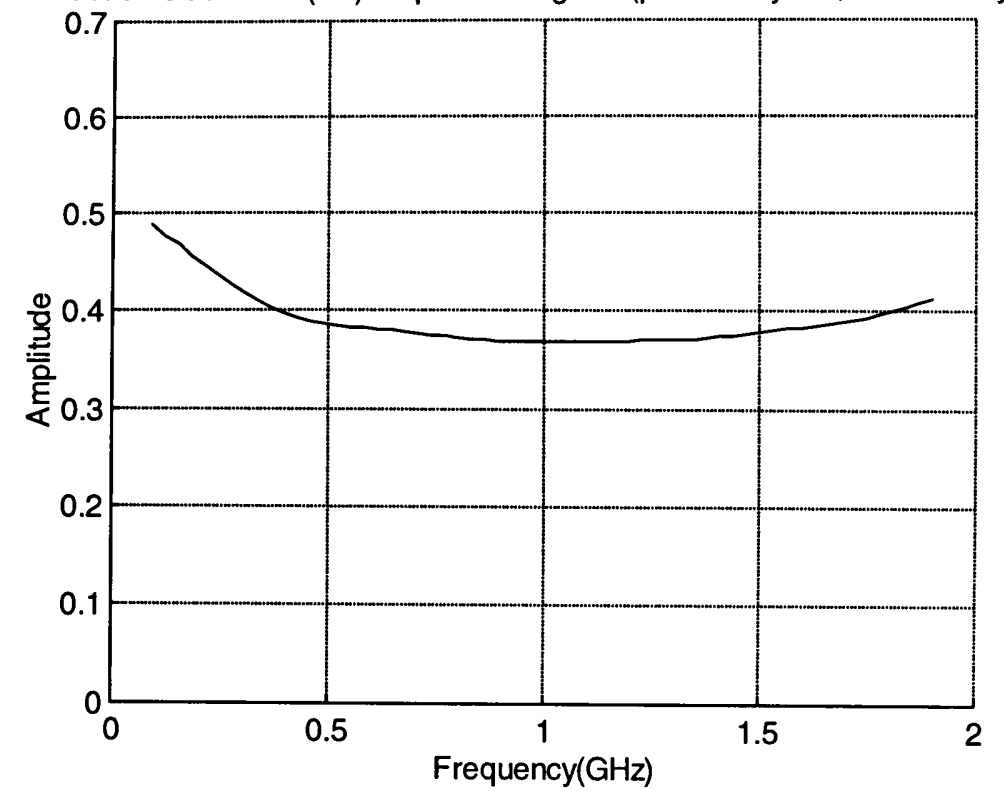

Figure 3.48 Soft diffraction coefficient of wedge $\left(\varepsilon_{r}, \sigma=12,0.1\right)$ for $\phi=35^{\circ}$ using FDTD

2) Hard Diffraction Coefficients $D_{h}$ for Wedge $(12,0.1)$ in $\phi=35^{\circ}$

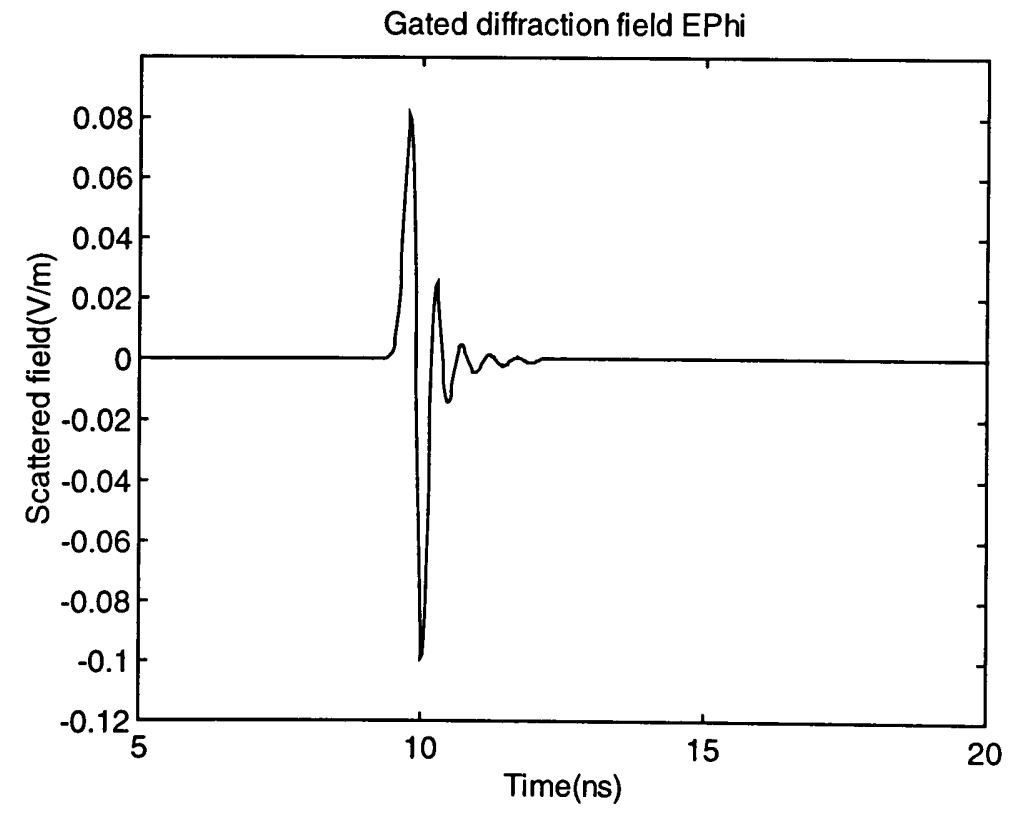

Figure 3.49 Diffraction field from $\phi$-polarized plane wave incident on wedge $\left(\varepsilon_{r}, \sigma=12,0.1\right)$ for $\phi=35^{\circ}$ using time-gating 


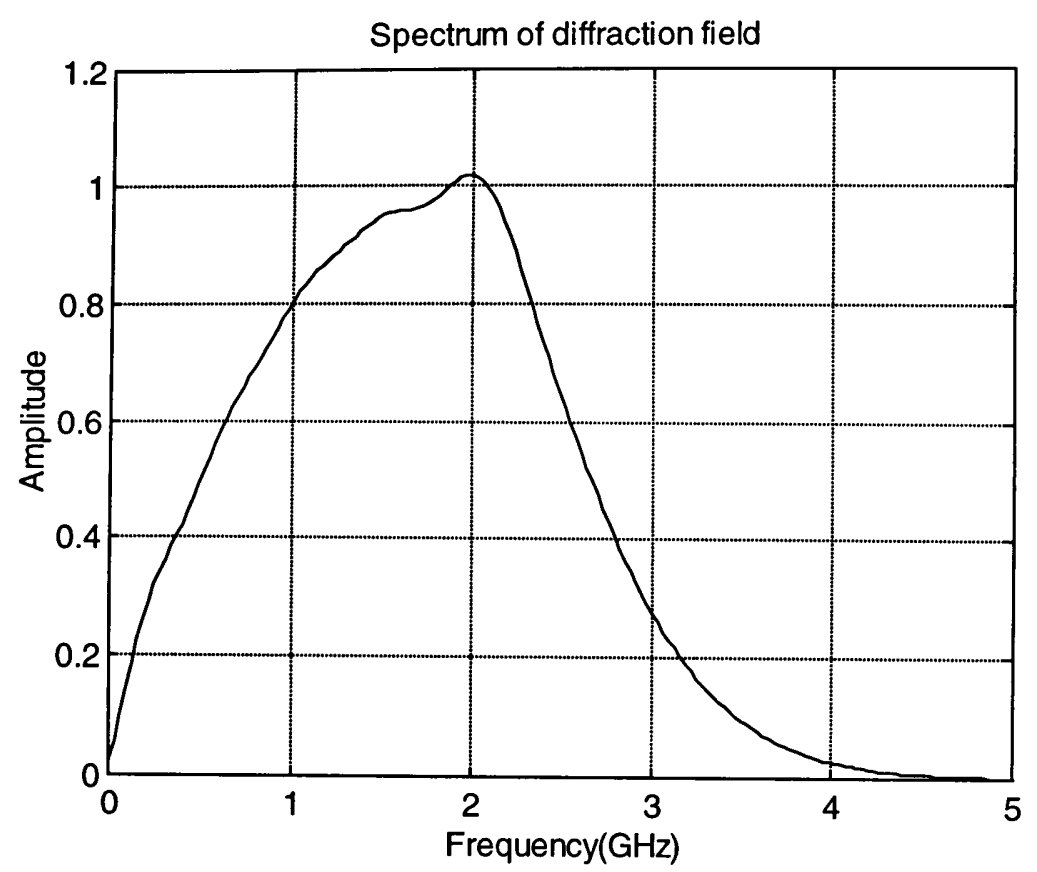

Figure 3.50 Spectrum of the diffraction field from $\phi$-polarized plane wave incident on wedge $\left(\varepsilon_{r}, \sigma=12,0.1\right)$ for $\phi=35^{\circ}$

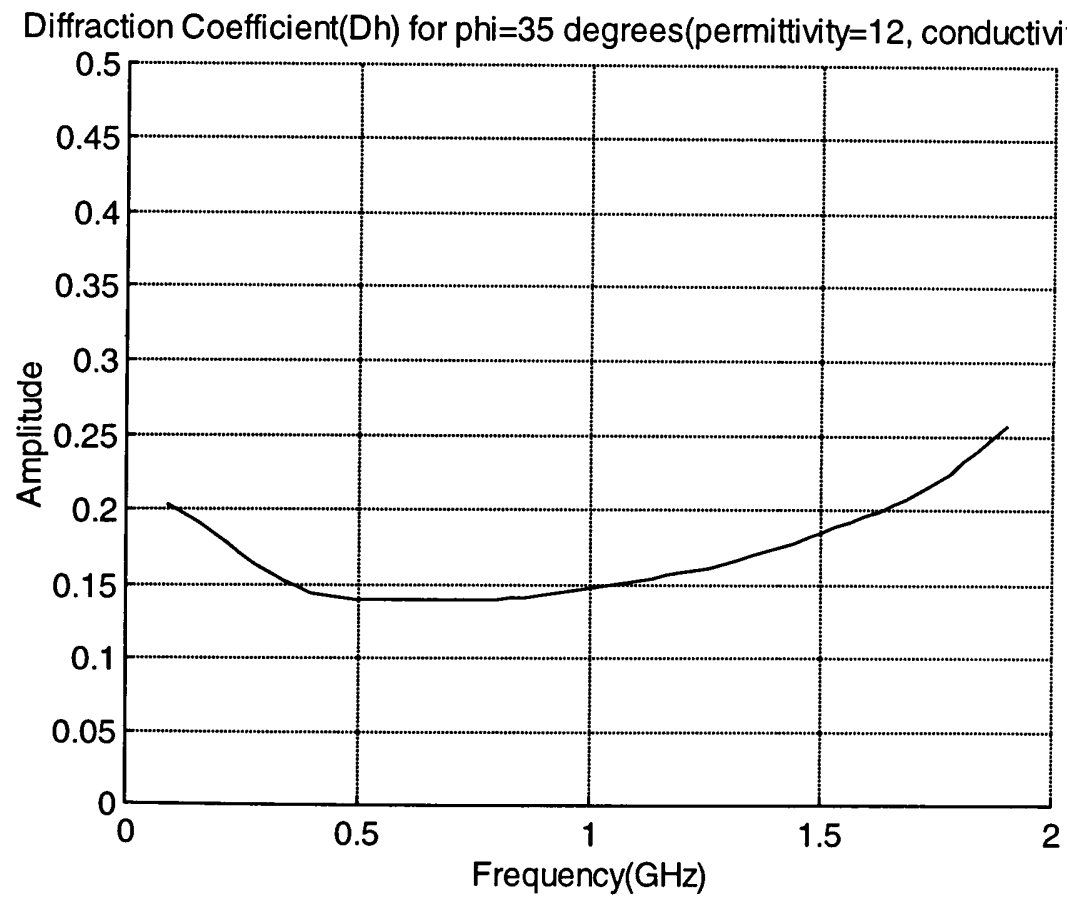

Figure 3.51 Hard diffraction coefficient of wedge $\left(\varepsilon_{r}, \sigma=12,0.1\right)$ for $\phi=35^{\circ}$ using FDTD 
3.4.1.2 Diffraction Coefficients for Wedge $\left(\varepsilon_{r}, \sigma=3,0.01\right)$ in $\phi=35^{\circ}$

1) Soft Diffraction Coefficients $D_{s}$ for Wedge $\left(\varepsilon_{r}, \sigma=3,0.01\right)$ in $\phi=35^{\circ}$

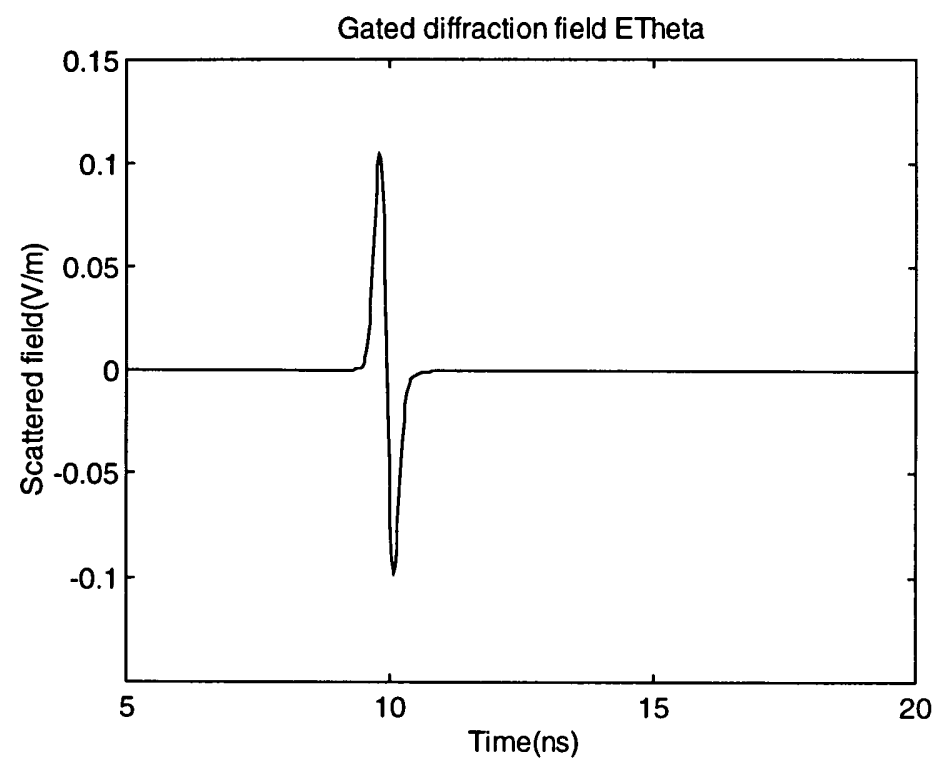

Figure 3.52 Diffraction field from $\theta$-polarized plane wave incident on wedge $\left(\varepsilon_{r}, \sigma=3,0.01\right)$ for $\phi=35^{\circ}$ using time-gating

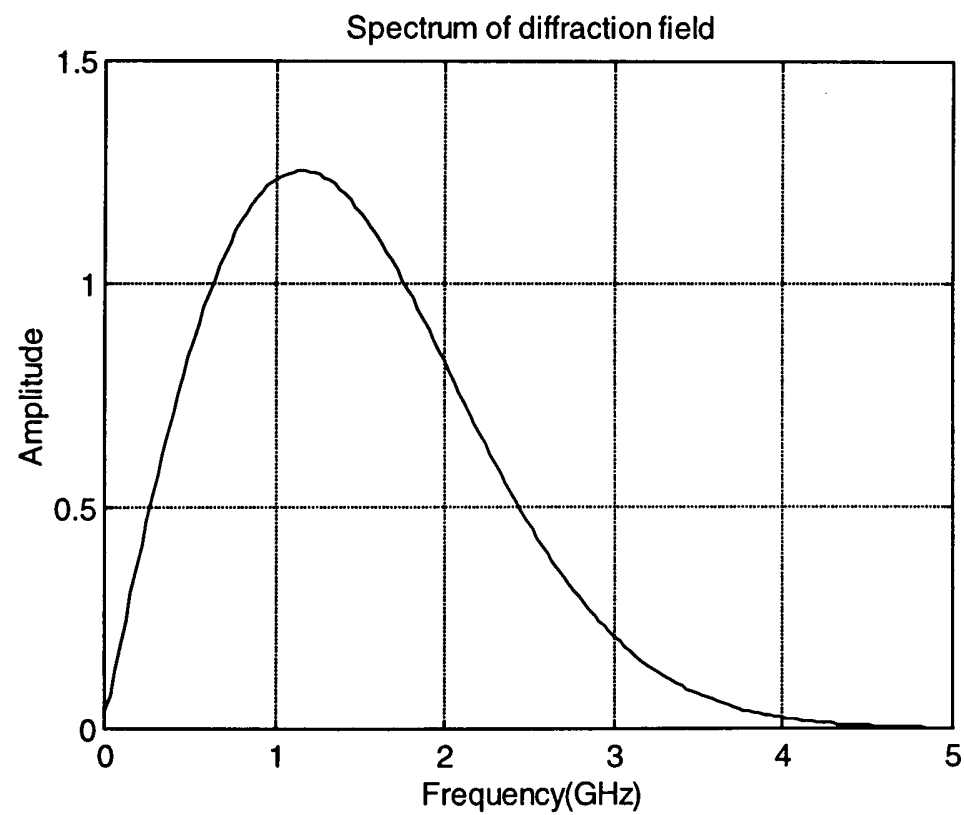

Figure 3.53 Spectrum of the diffraction field from $\theta$-polarized plane wave incident on wedge $\left(\varepsilon_{r}, \sigma=3,0.01\right)$ for $\phi=35^{\circ}$ 
Diffraction Coefficient(Ds) for phi=35 degrees(permittivity $=3$, conductivity $=0.01$ )

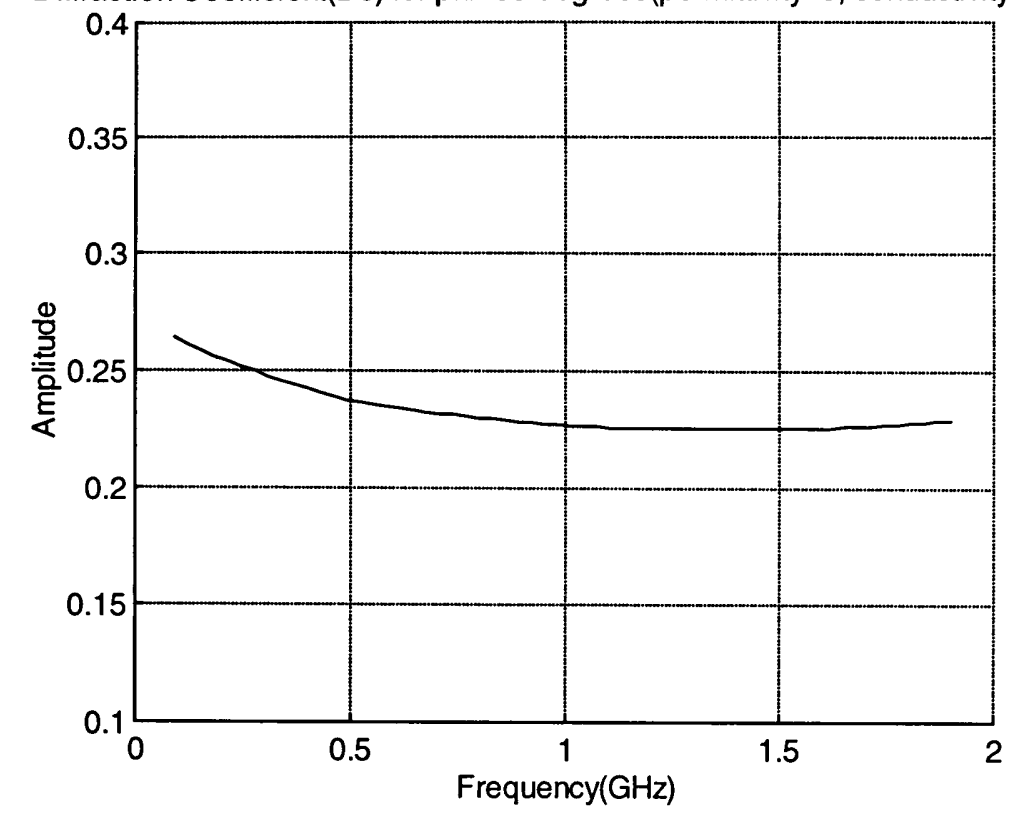

Figure 3.54 Soft diffraction coefficient of wedge $\left(\varepsilon_{r}, \sigma=3,0.01\right)$ for $\phi=35^{\circ}$ using FDTD

2) Hard Diffraction Coefficients $D_{h}$ for Wedge $(3,0.01)$ in $\phi=35^{\circ}$

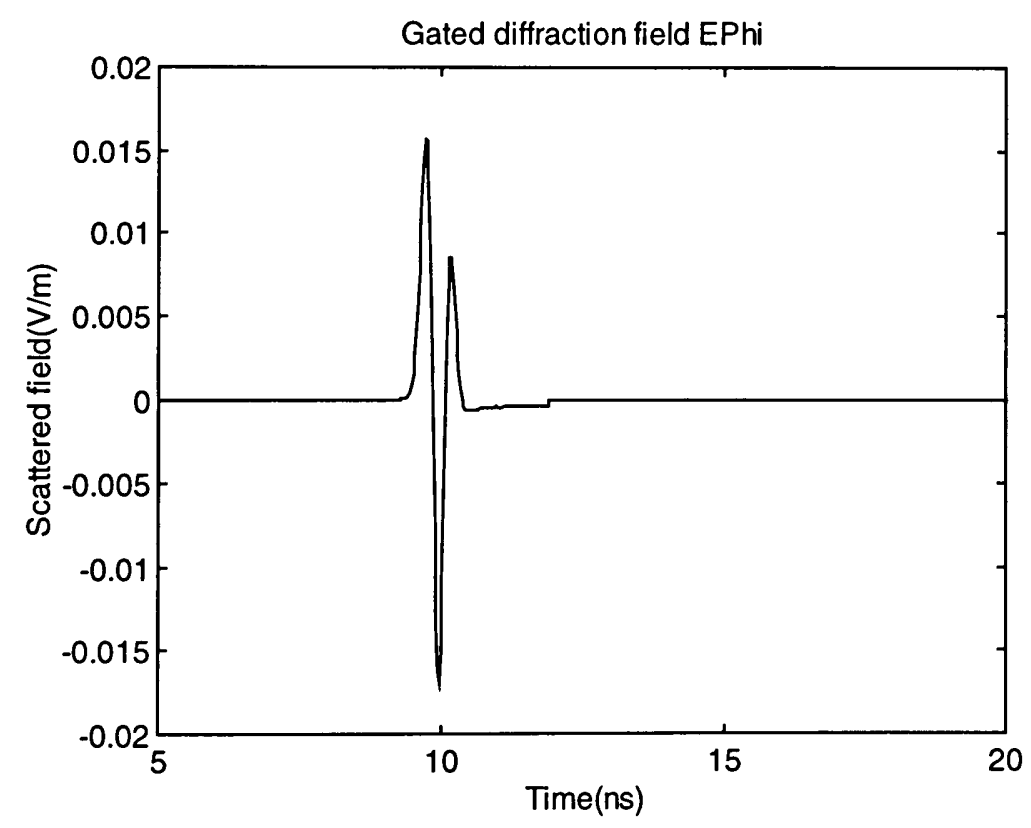

Figure 3.55 Diffraction field from $\phi$-polarized plane wave incident on wedge $\left(\varepsilon_{r}, \sigma=3,0.01\right)$ for $\phi=35^{\circ}$ using time-gating 


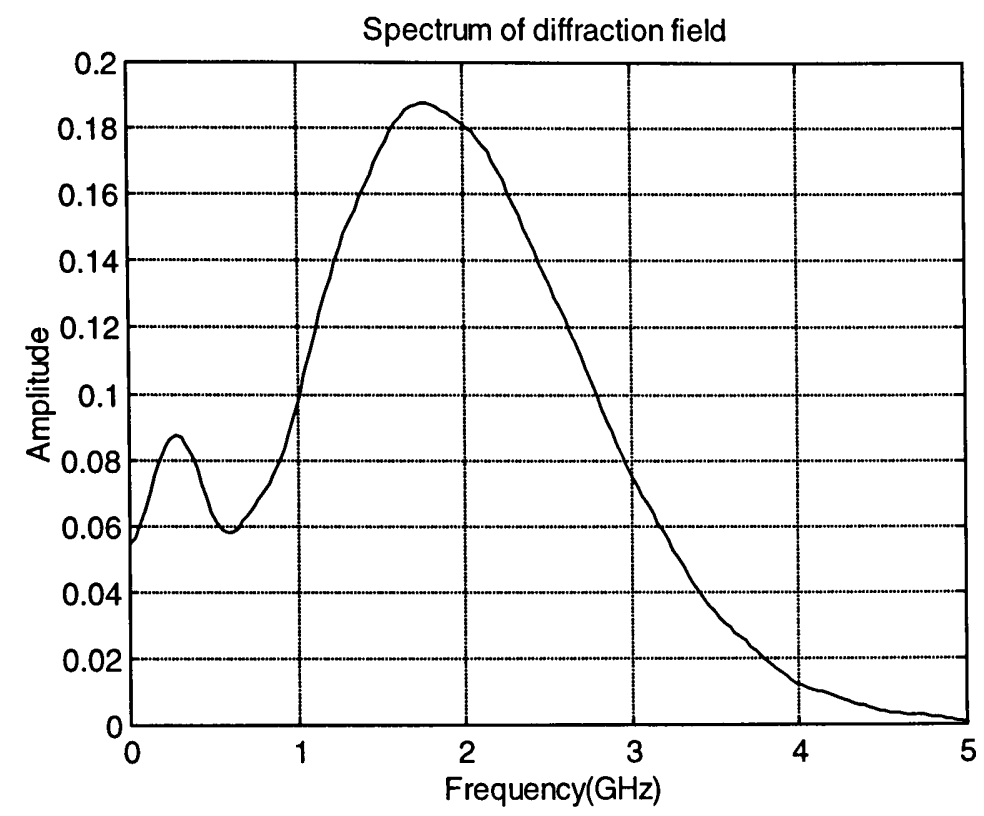

Figure 3.56 Spectrum of the diffraction field from $\phi$-polarized plane wave incident on wedge $\left(\varepsilon_{r}, \sigma=3,0.01\right)$ for $\phi=35^{\circ}$

Diffraction Coefficient(Dh) for phi $=35$ degrees (permittivity $=3$, conductivity $=0.01$ )

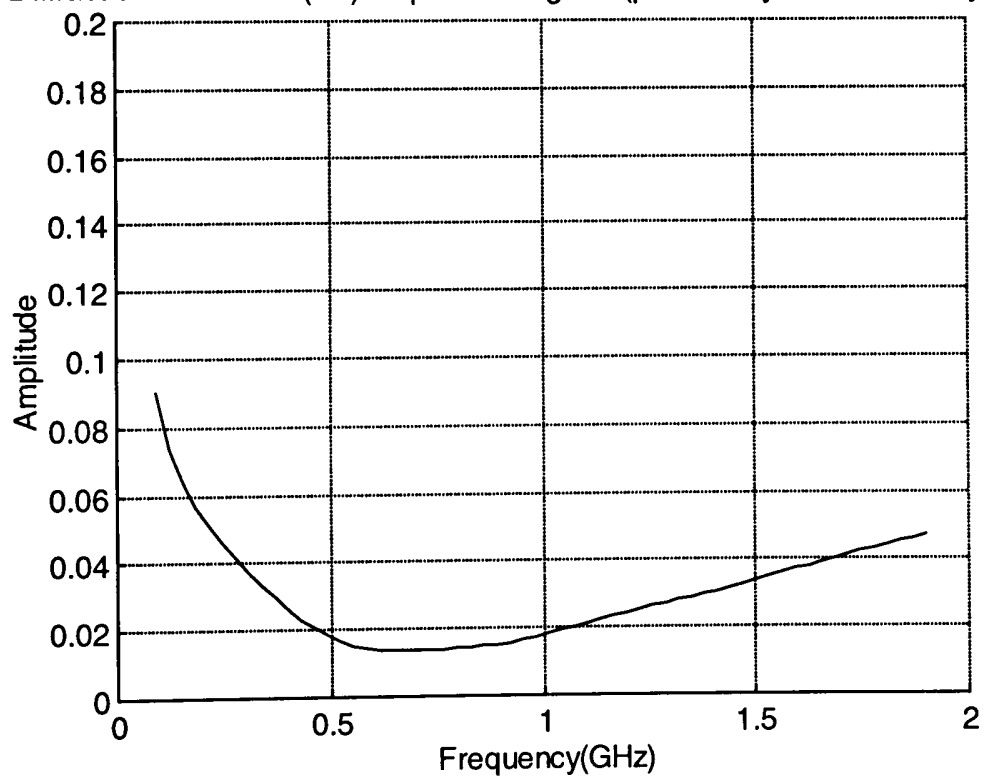

Figure 3.57 Hard diffraction coefficient of wedge $\left(\varepsilon_{r}, \sigma=3,0.01\right)$ for $\phi=35^{\circ}$ using FDTD 
Compare soft and hard diffraction coefficients $D_{s}$ and $D_{h}$ of the four wedge materials.

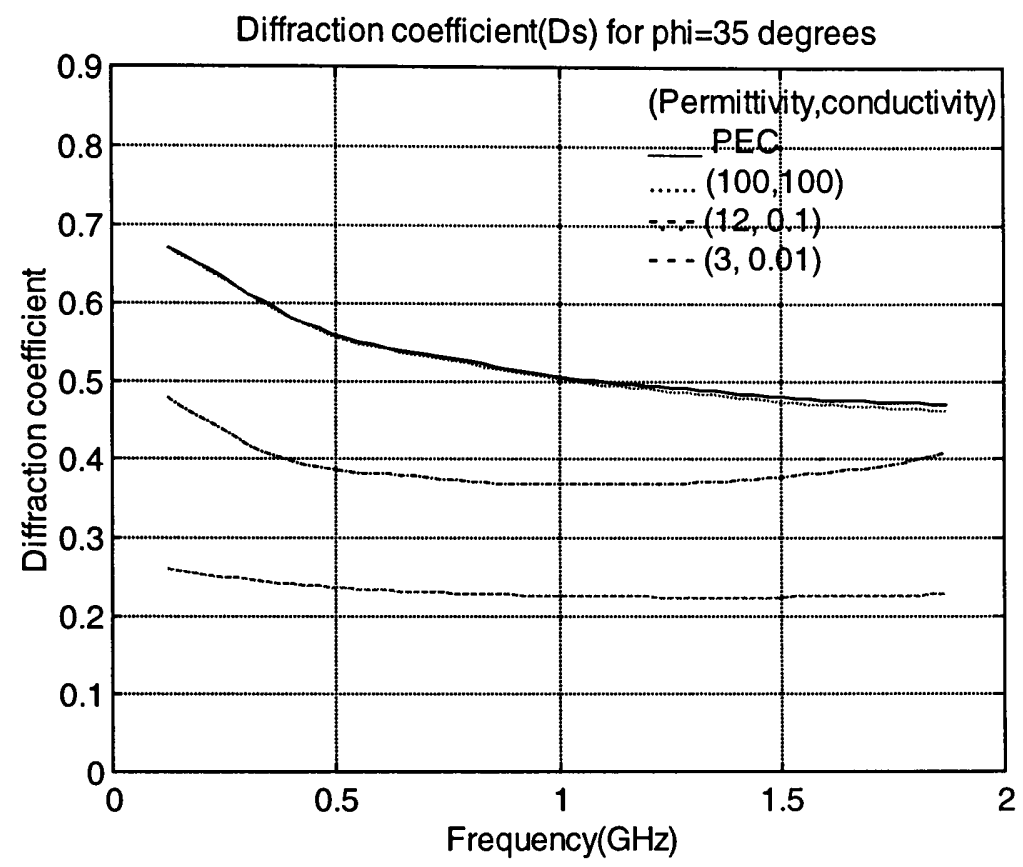

Figure 3.58 3-Dimensional soft diffraction coefficient for four wedge types for $\phi=35^{\circ}$

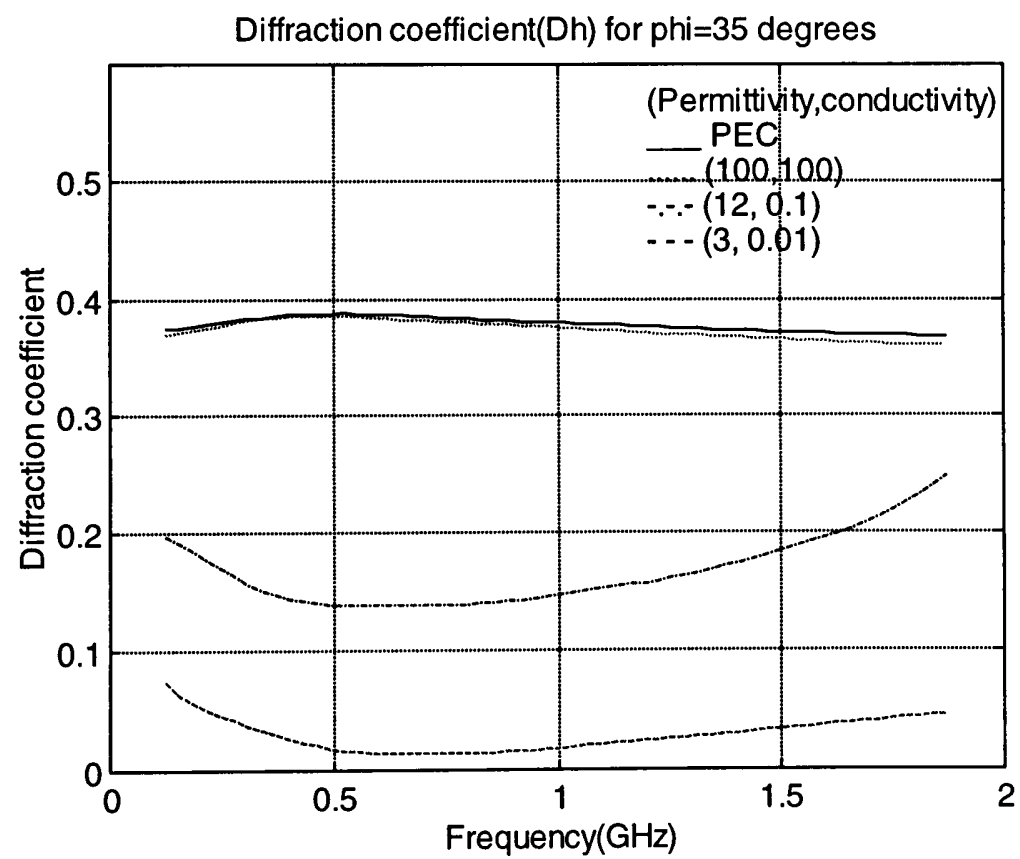

Figure 3.59 3-Dimensional hard diffraction coefficient for four wedge types for $\phi=35^{\circ}$ 
3.4.2 Diffraction Coefficients of Dielectric Wedge for $\phi^{\prime}=150^{\circ}, \beta^{\prime}=70^{\circ}, \phi=40^{\circ}$

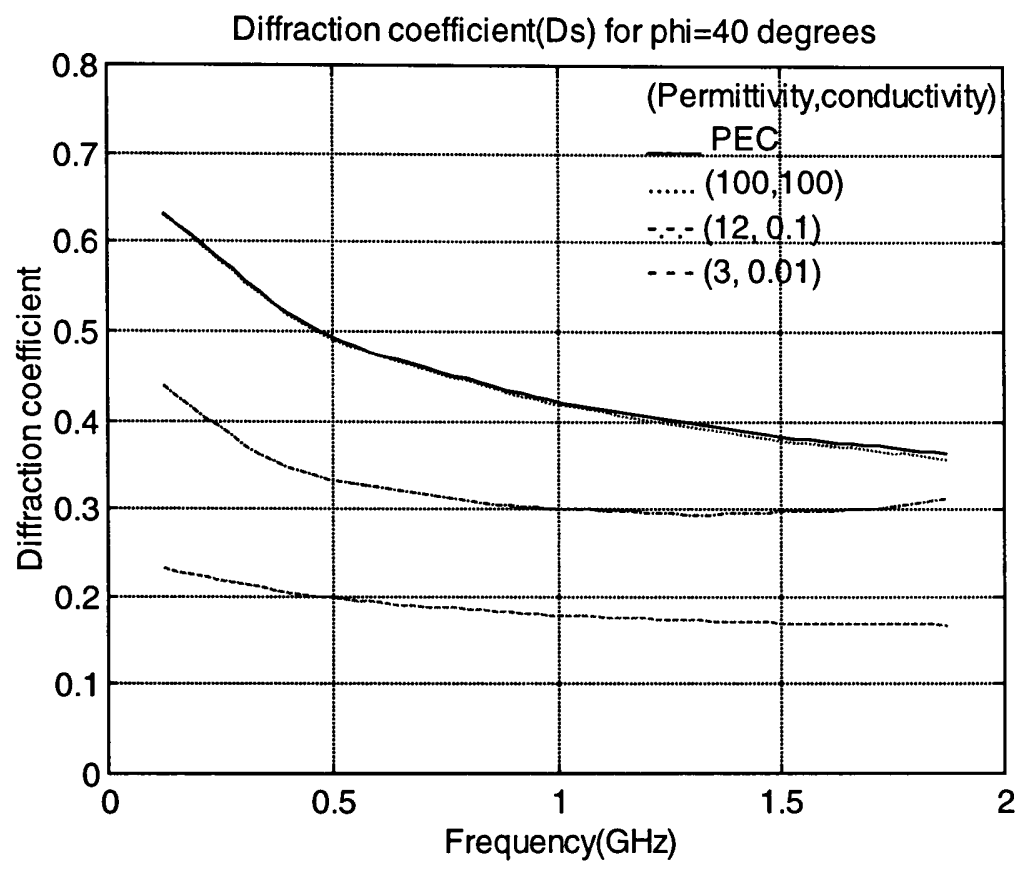

Figure 3.60 3-Dimensional soft diffraction coefficient for four wedge types for $\phi=40^{\circ}$

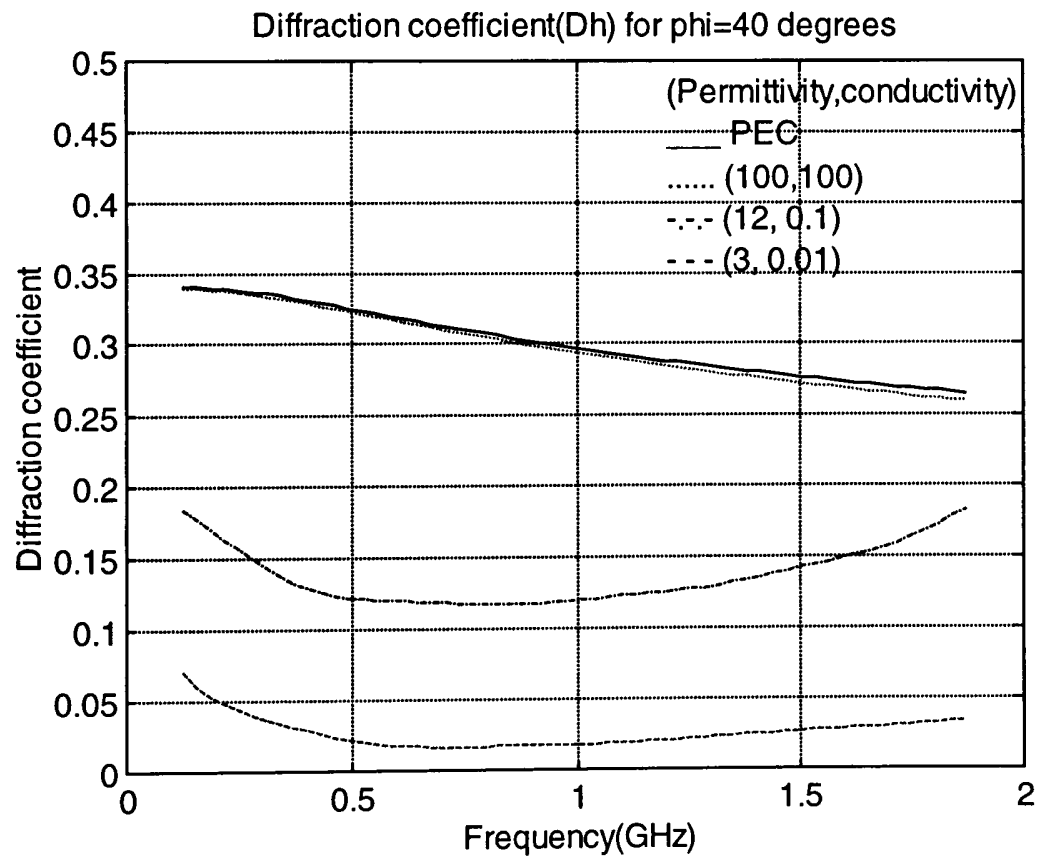

Figure 3.61 3-Dimensional hard diffraction coefficient for four wedge types for $\phi=40^{\circ}$ 
3.4.3 Diffraction Coefficients of Dielectric Wedge for $\phi^{\prime}=150^{\circ}, \beta^{\prime}=70^{\circ}, \phi=45^{\circ}$

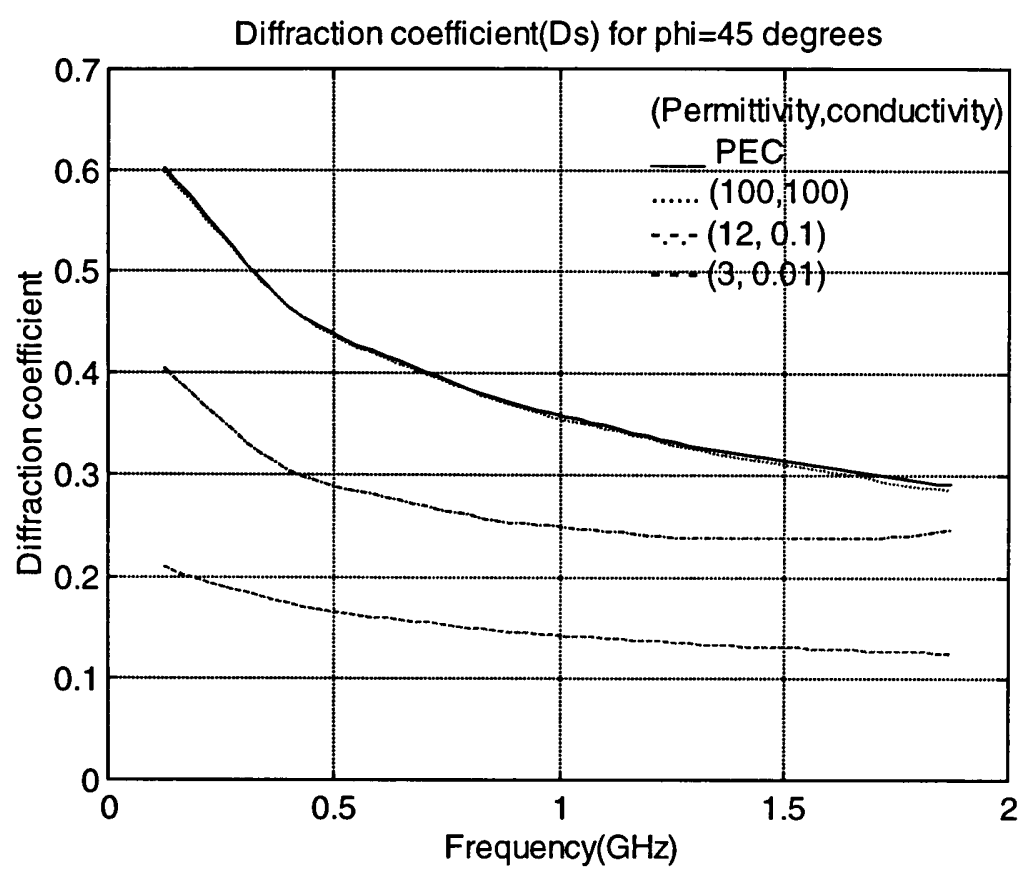

Figure 3.62 3-Dimensional soft diffraction coefficient for four wedge types for $\phi=45^{\circ}$

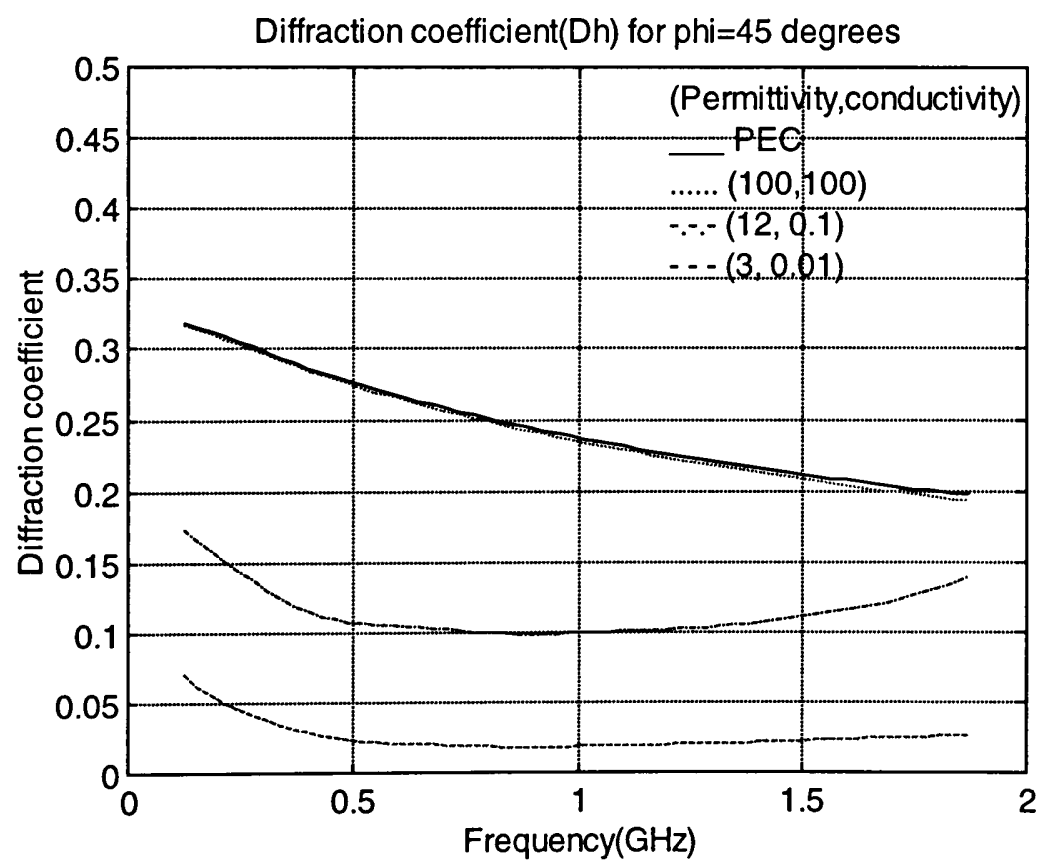

Figure 3.63 3-Dimensional hard diffraction coefficient for four wedge types for $\phi=45^{\circ}$ 
3.4.4 Diffraction Coefficients of Dielectric Wedge for $\phi^{\prime}=150^{\circ}, \beta^{\prime}=\mathbf{7 0}^{\circ}, \phi=50^{\circ}$

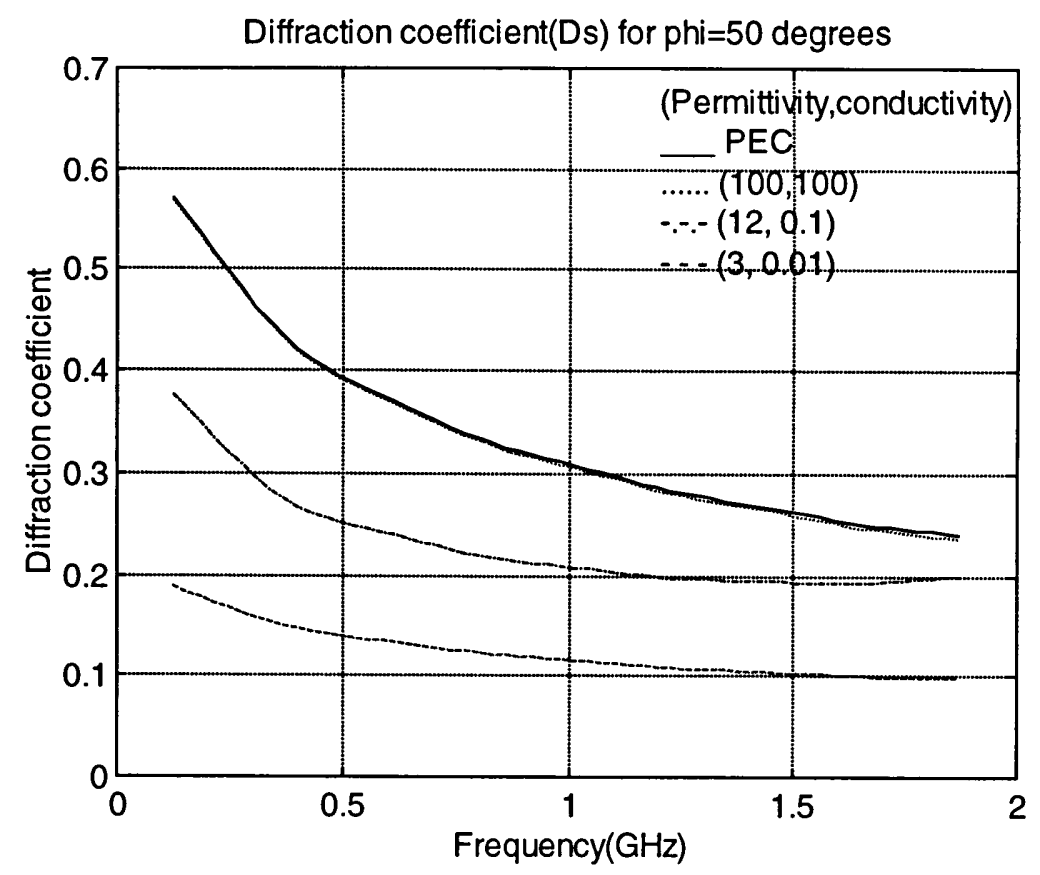

Figure 3.64 3-Dimensional soft diffraction coefficient for four wedge types for $\phi=50^{\circ}$

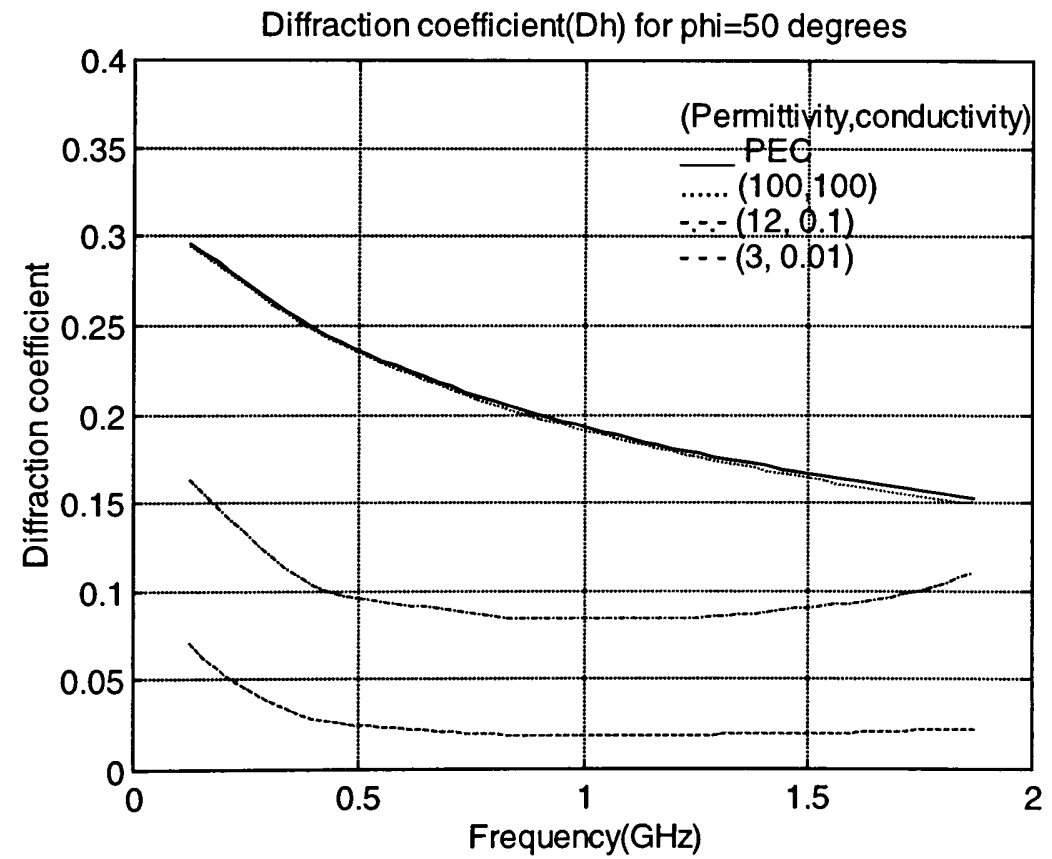

Figure 3.65 3-Dimensional hard diffraction coefficient for four wedge types for $\phi=50^{\circ}$ 
3.4.5 Diffraction Coefficients of Dielectric Wedge for $\phi^{\prime}=150^{\circ}, \beta^{\prime}=70^{\circ}, \phi=60^{\circ}$

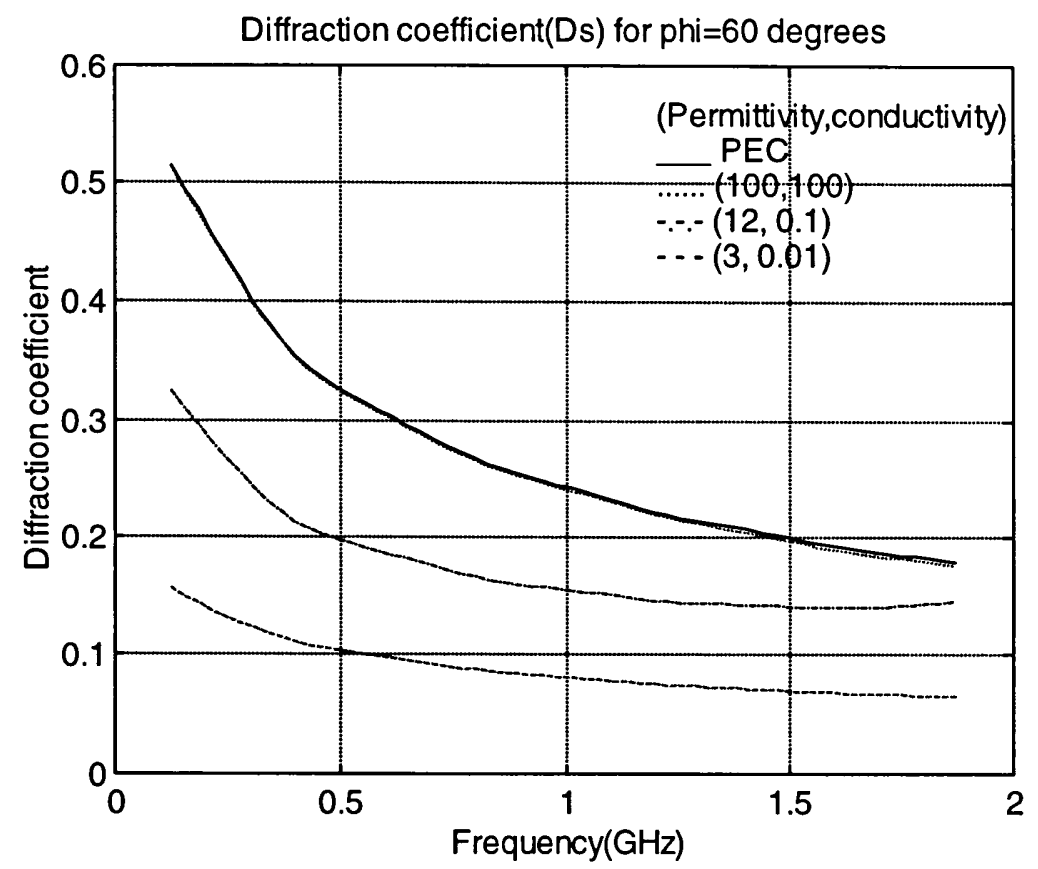

Figure 3.66 3-Dimensional soft diffraction coefficient for four wedge types for $\phi=60^{\circ}$

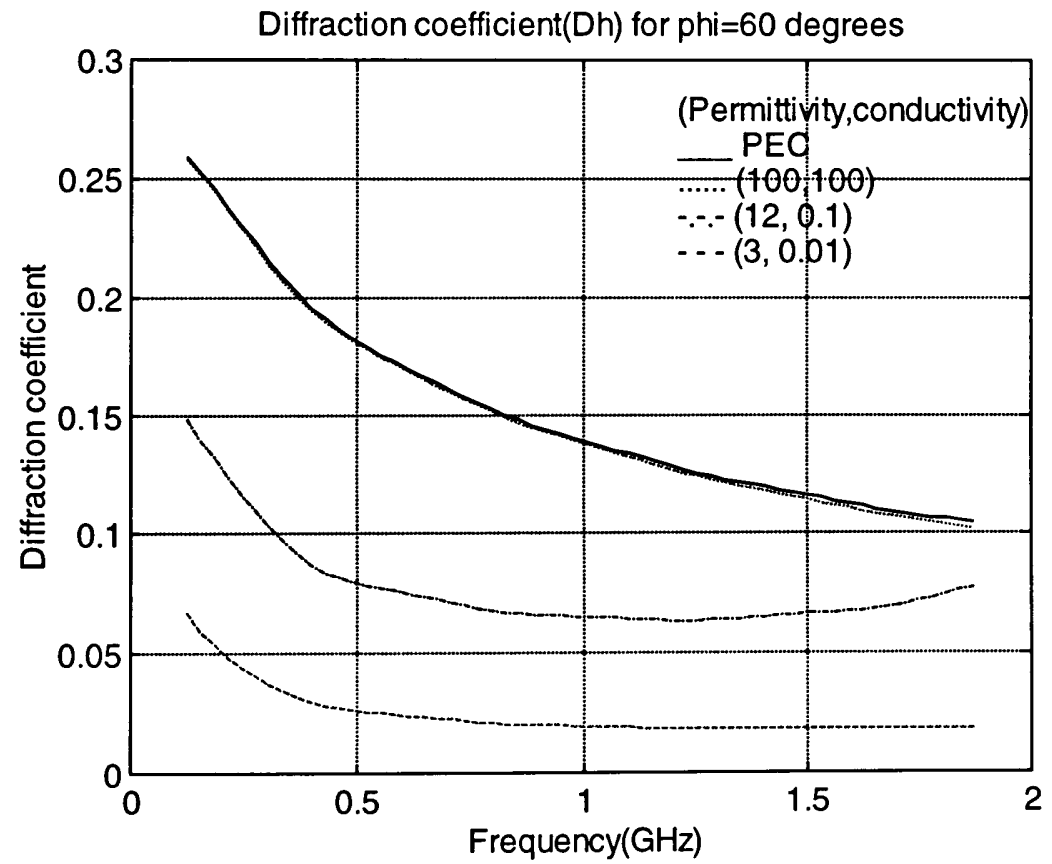

Figure 3.67 3-Dimensional hard diffraction coefficient for four wedge types for $\phi=60^{\circ}$ 
3.4.6 Diffraction Coefficients of Dielectric Wedge for $\phi^{\prime}=150^{\circ}, \beta^{\prime}=\mathbf{7 0}^{\circ}, \phi=70^{\circ}$

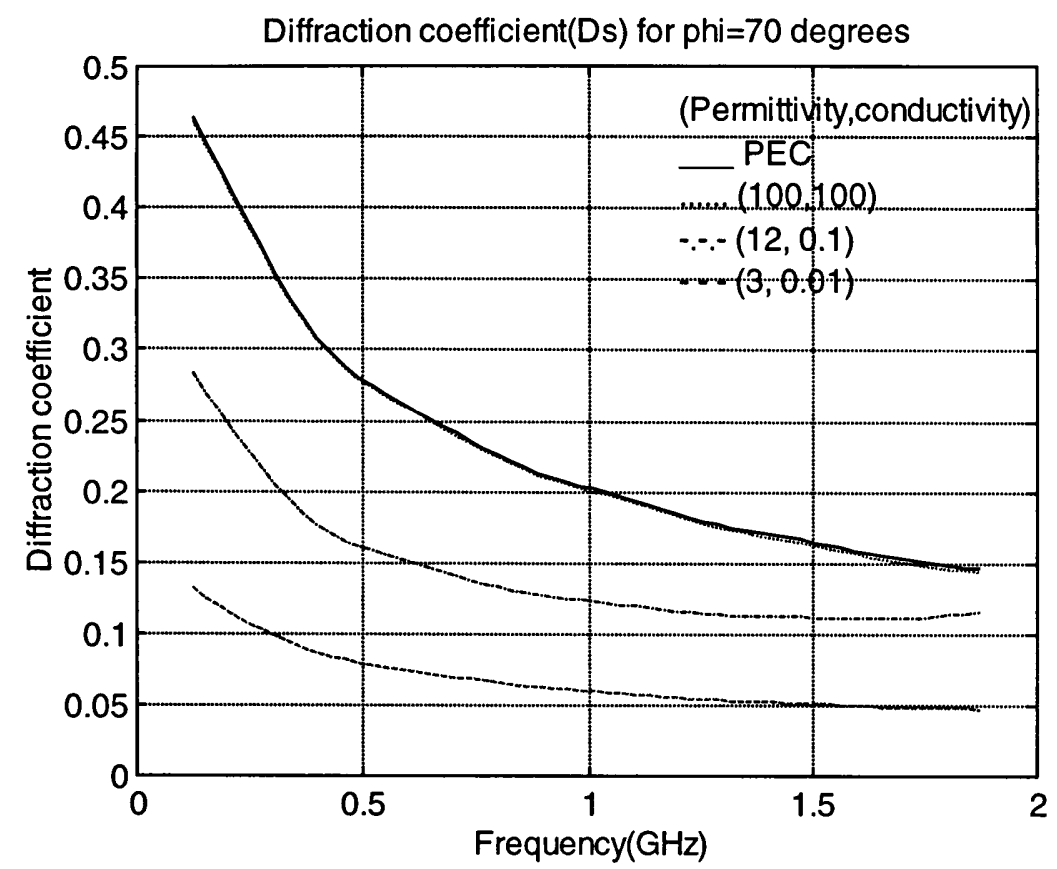

Figure 3.68 3-Dimensional soft diffraction coefficient for four wedge types for $\phi=70^{\circ}$

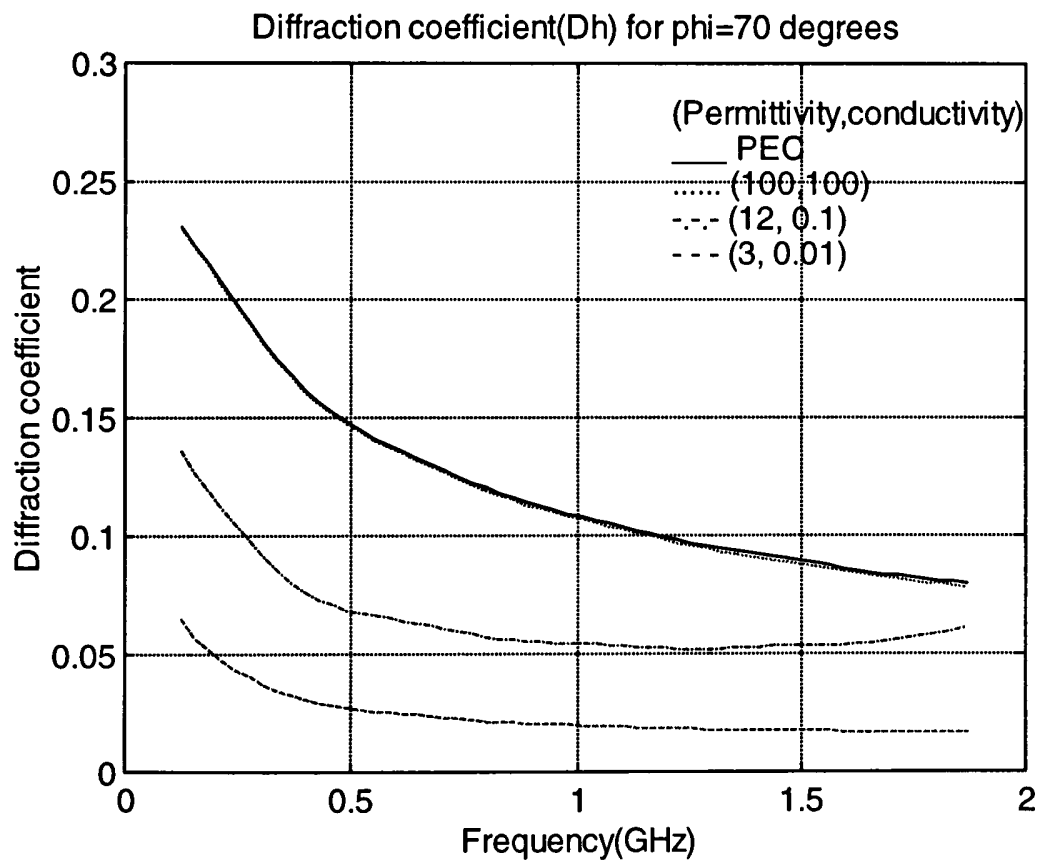

Figure 3.69 3-Dimensional hard diffraction coefficient for four wedge types for $\phi=70^{\circ}$ 
3.4.7 Diffraction Coefficients of Dielectric Wedge for $\phi^{\prime}=150^{\circ}, \beta^{\prime}=70^{\circ}, \phi=80^{\circ}$

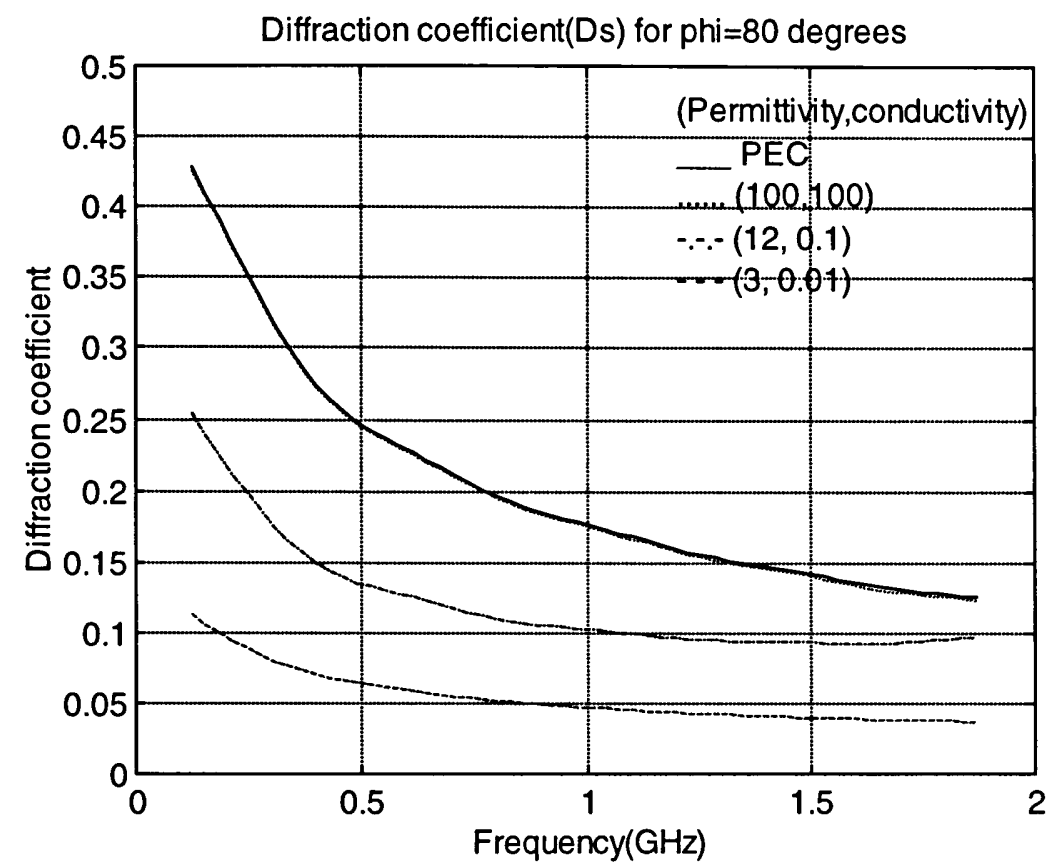

Figure 3.70 3-Dimensional soft diffraction coefficient for four wedge types for $\phi=80^{\circ}$

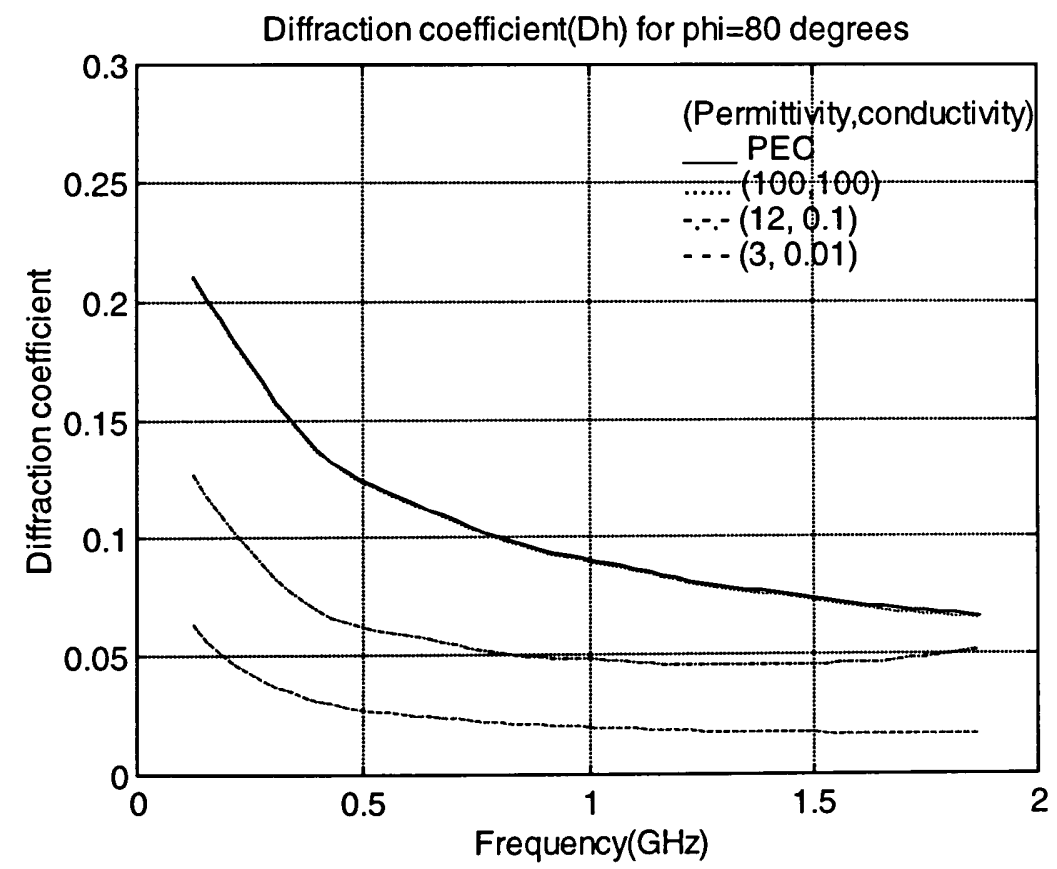

Figure 3.71 3-Dimensional soft diffraction coefficient for four wedge types for $\phi=80^{\circ}$ 
3.4.8 Diffraction Coefficients of Dielectric Wedge for $\phi^{\prime}=150^{\circ}, \beta^{\prime}=\mathbf{7 0}^{\circ}, \phi=100^{\circ}$

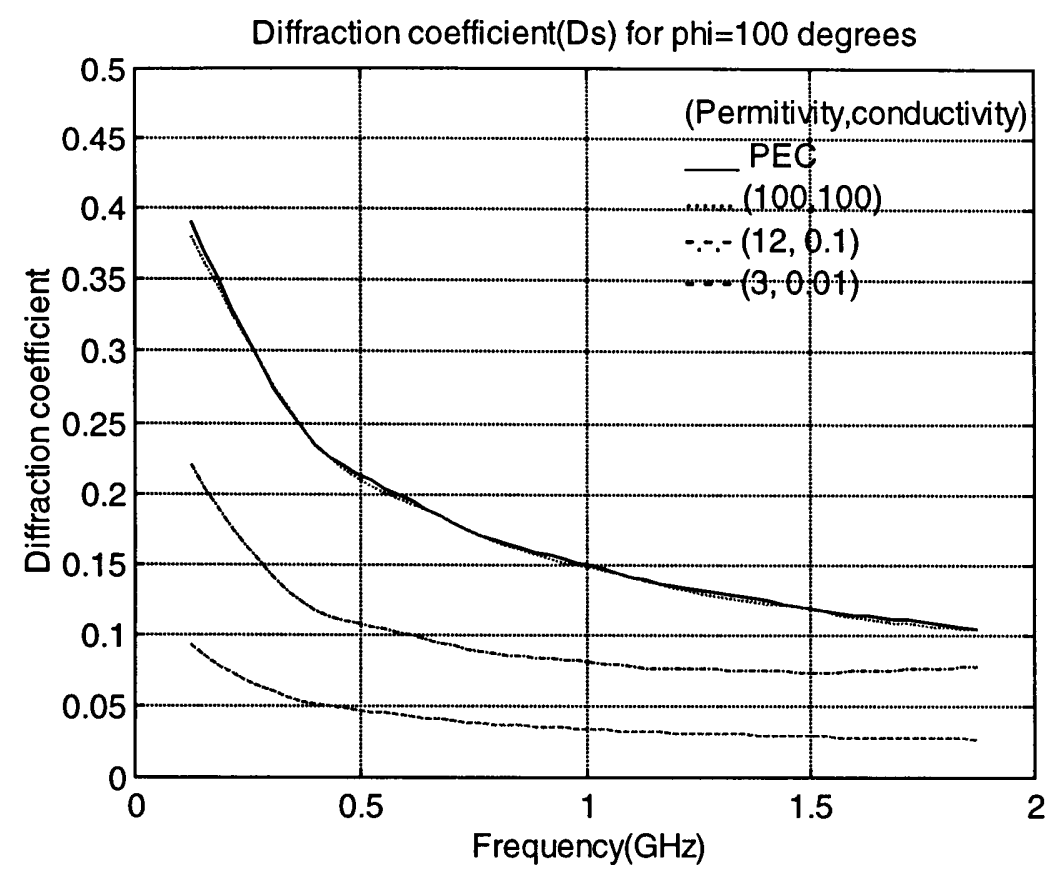

Figure 3.72 3-Dimensional soft diffraction coefficient for four wedge types for $\phi=100^{\circ}$

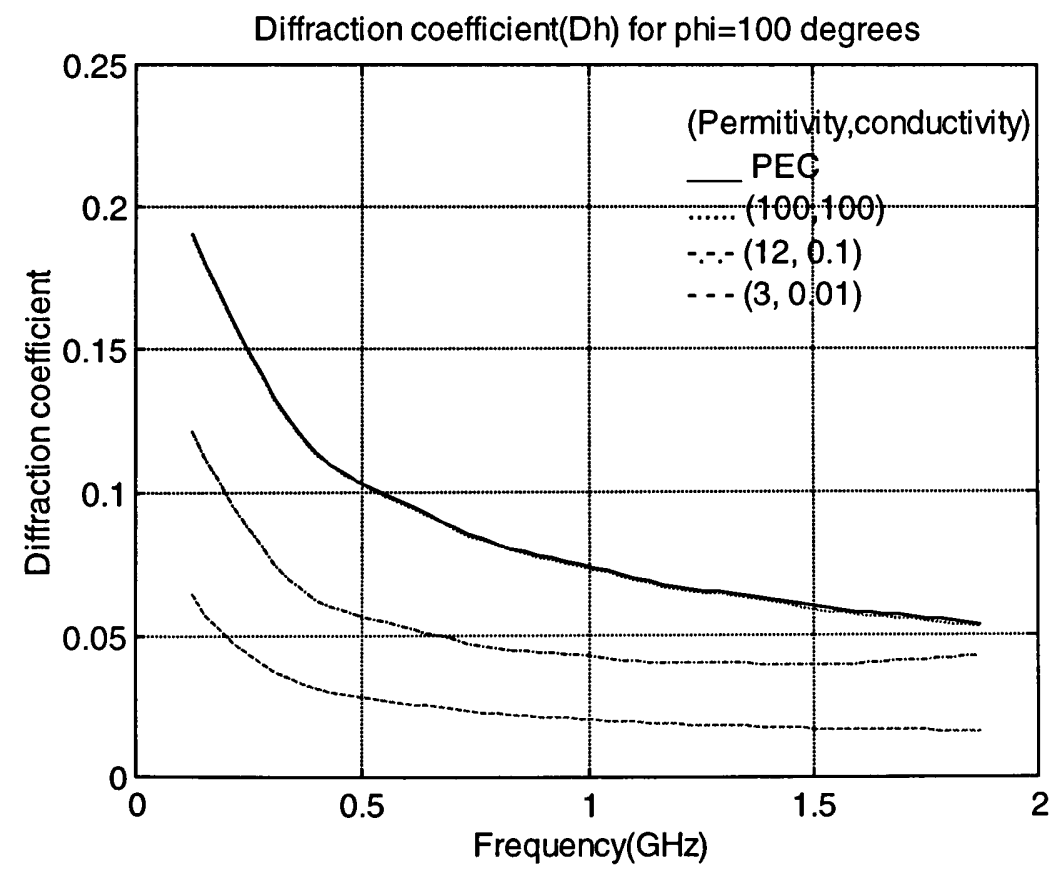

Figure 3.73 3-Dimensional soft diffraction coefficient for four wedge types for $\phi=100^{\circ}$ 
Table 3.1 Soft diffraction coefficients in $1.06 \mathrm{~m}$ of diffraction angles

for four wedge types $\left(\beta^{\prime}=70^{\circ}, \phi^{\prime}=150^{\circ}\right)$

\begin{tabular}{|c|c|c|c|c|c|c|c|c|}
\hline \multicolumn{9}{|c|}{ Theta Polarization $\left(D_{s}\right)$} \\
\hline \multirow{2}{*}{$\begin{array}{c}\text { Diffraction } \\
\text { Angle } \phi\end{array}$} & \multicolumn{9}{|c|}{$\mathbf{8 5 0} \mathbf{M H z}$} & \multicolumn{4}{c|}{$\mathbf{1 . 7 G H z}$} \\
\cline { 2 - 9 } & PEC & $(100,100)$ & $(12,0.1)$ & $(3,0.01)$ & PEC & $(100,100)$ & $(12,0.1)$ & $(3,0.01)$ \\
\hline $35^{\circ}$ & 0.5183 & 0.5153 & 0.3700 & 0.2292 & 0.4734 & 0.4660 & 0.3913 & 0.2270 \\
\hline $40^{\circ}$ & 0.4387 & 0.4361 & 0.3056 & 0.1833 & 0.3721 & 0.3663 & 0.3014 & 0.1685 \\
\hline $45^{\circ}$ & 0.3750 & 0.3731 & 0.2552 & 0.1479 & 0.2987 & 0.2945 & 0.2383 & 0.1278 \\
\hline $50^{\circ}$ & 0.3252 & 0.3231 & 0.2154 & 0.1203 & 0.2477 & 0.2437 & 0.1942 & 0.0991 \\
\hline $60^{\circ}$ & 0.2587 & 0.2569 & 0.1622 & 0.0849 & 0.1869 & 0.1838 & 0.1412 & 0.0664 \\
\hline $70^{\circ}$ & 0.2168 & 0.2152 & 0.1290 & 0.0636 & 0.1518 & 0.1492 & 0.1114 & 0.0485 \\
\hline $80^{\circ}$ & 0.1893 & 0.1878 & 0.1072 & 0.0500 & 0.1307 & 0.1285 & 0.0934 & 0.0380 \\
\hline $100^{\circ}$ & 0.1613 & 0.1593 & 0.0848 & 0.0362 & 0.1111 & 0.1079 & 0.0759 & 0.0278 \\
\hline
\end{tabular}

Table 3.2 Hard diffraction coefficients in $1.06 \mathrm{~m}$ of diffraction angles for four wedge types $\left(\beta^{\prime}=70^{\circ}, \phi^{\prime}=150^{\circ}\right)$

\begin{tabular}{|c|c|c|c|c|c|c|c|c|}
\hline \multicolumn{9}{|c|}{ Phi Polarization $\left(D_{h}\right)$} \\
\hline \multirow{2}{*}{$\begin{array}{c}\text { Diffraction } \\
\text { Angle } \phi\end{array}$} & \multicolumn{9}{|c|}{$\mathbf{8 5 0} \mathbf{M H z}$} & \multicolumn{4}{c|}{$\mathbf{1 . 7 G H z}$} \\
\cline { 2 - 9 } & PEC & $(100,100)$ & $(12,0.1)$ & $(3,0.01)$ & PEC & $(100,100)$ & $(12,0.1)$ & $(3,0.01)$ \\
\hline $35^{\circ}$ & 0.3816 & 0.3783 & 0.1409 & 0.0148 & 0.3695 & 0.3617 & 0.2124 & 0.0413 \\
\hline $40^{\circ}$ & 0.3041 & 0.3012 & 0.1169 & 0.0171 & 0.2690 & 0.2638 & 0.1590 & 0.0306 \\
\hline $45^{\circ}$ & 0.2463 & 0.2440 & 0.0986 & 0.0181 & 0.2026 & 0.1988 & 0.1228 & 0.0248 \\
\hline $50^{\circ}$ & 0.2025 & 0.2006 & 0.0845 & 0.0191 & 0.1580 & 0.1550 & 0.0982 & 0.0215 \\
\hline $60^{\circ}$ & 0.1475 & 0.1463 & 0.0665 & 0.0203 & 0.1085 & 0.1066 & 0.0706 & 0.0182 \\
\hline $70^{\circ}$ & 0.1159 & 0.1149 & 0.0559 & 0.0208 & 0.0832 & 0.0817 & 0.0561 & 0.0170 \\
\hline $80^{\circ}$ & 0.0965 & 0.0957 & 0.0496 & 0.0210 & 0.0687 & 0.0675 & 0.0480 & 0.0164 \\
\hline $100^{\circ}$ & 0.0790 & 0.0784 & 0.0446 & 0.0218 & 0.0565 & 0.0555 & 0.0411 & 0.0167 \\
\hline
\end{tabular}

For wedge $\left(\varepsilon_{r}, \sigma=100,100\right)$ in frequency band $0.1 \sim 2 \mathrm{GHz}$

$$
\omega \varepsilon=(0.1 \sim 2) \times 10^{9} \times \varepsilon_{0} \times 100=0.56 \sim 11.1<<\sigma=100
$$

is classified as a conductor and behaves similar to the PEC from above results.

For wedge $\left(\varepsilon_{r}, \sigma=12,0.1\right), \omega \varepsilon=0.067 \sim 1.33$

and for wedge $\left(\varepsilon_{r}, \sigma=3,0.01\right), \omega \varepsilon=0.017 \sim 0.33$

are classified as quasiconductor when $\omega \varepsilon \sim \sigma$ and as dielectric when $\omega \varepsilon \gg>\sigma$. 
We draw the following figures to compare the data from tables 3.1 and 3.2.

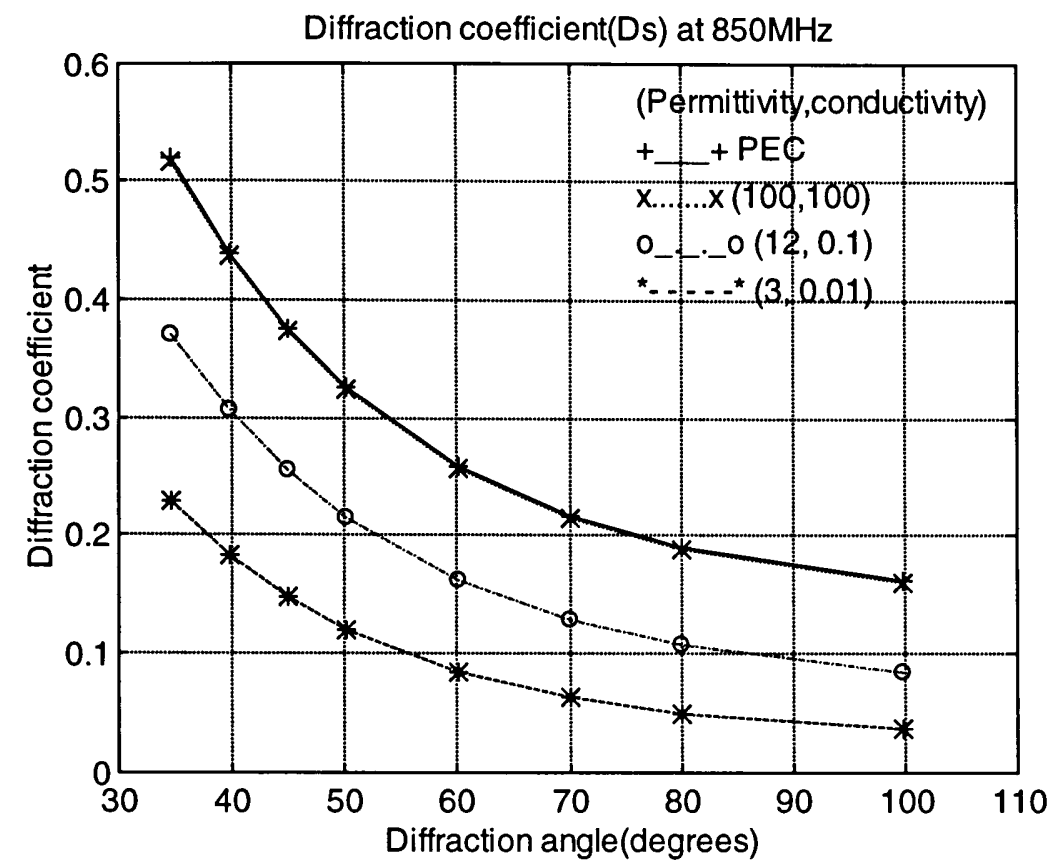

Figure 3.74 Comparison of soft diffraction coefficients at $850 \mathrm{MHz}$ to different directions for four wedge types at $1.06 \mathrm{~m}\left(\beta^{\prime}=70^{\circ}, \phi^{\prime}=150^{\circ}\right)$ by asymptotic and FDTD

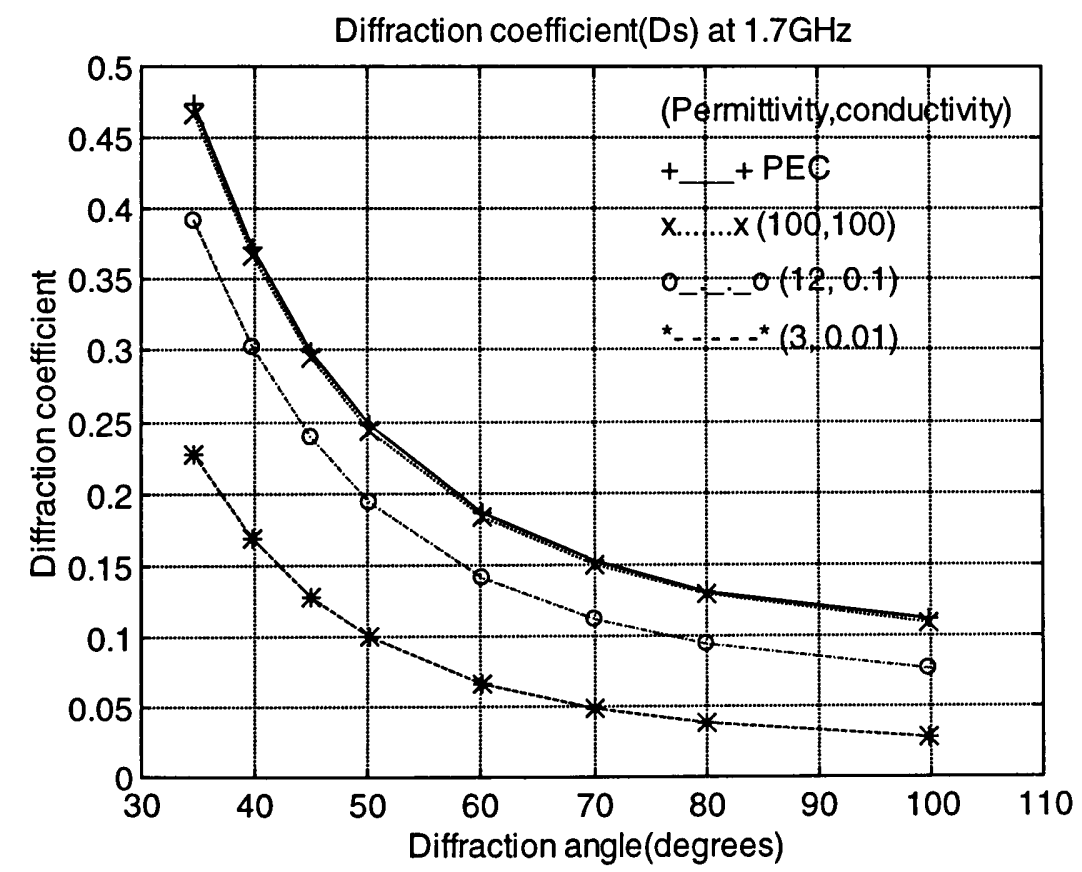

Figure 3.75 Comparison of soft diffraction coefficients at $1.7 \mathrm{GHz}$ to different directions for four wedge types at $1.06 \mathrm{~m}\left(\beta^{\prime}=70^{\circ}, \phi^{\prime}=150^{\circ}\right)$ by asymptotic and FDTD 


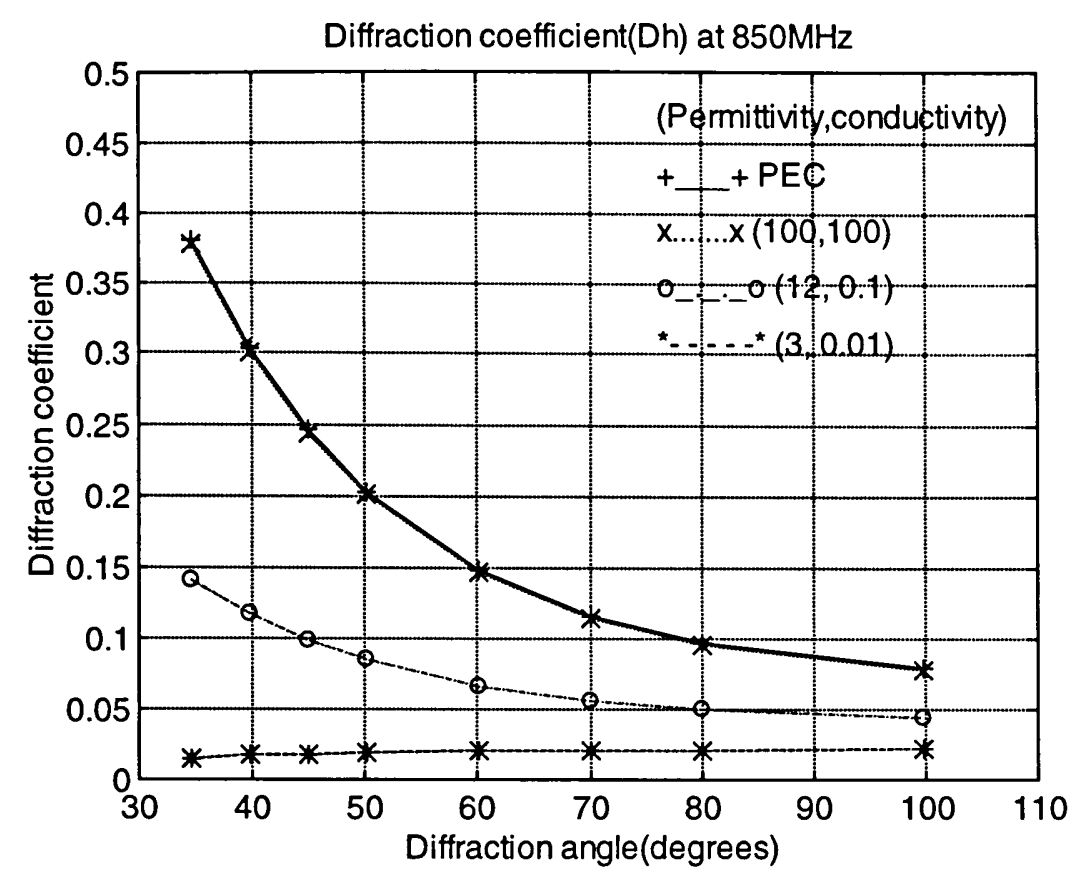

Figure 3.76 Comparison of hard diffraction coefficients at $850 \mathrm{MHz}$ to different directions for four wedge types at $1.06 \mathrm{~m}\left(\beta^{\prime}=70^{\circ}, \phi^{\prime}=150^{\circ}\right)$ by asymptotic and FDTD

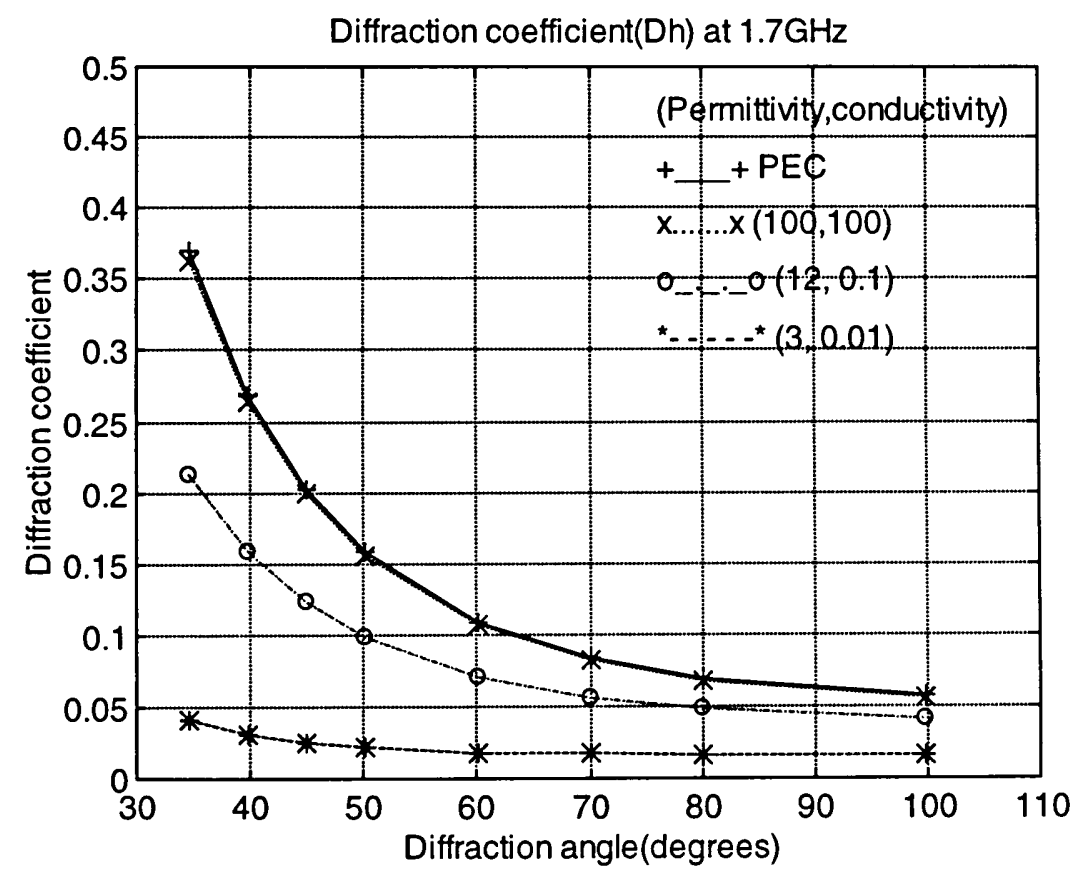

Figure 3.77 Comparison of hard diffraction coefficients at $1.7 \mathrm{GHz}$ to different directions for four wedge types at $1.06 \mathrm{~m}\left(\beta^{\prime}=70^{\circ}, \phi^{\prime}=150^{\circ}\right)$ by asymptotic and FDTD 


\section{Chapter 4}

\section{2-Dimensional Diffraction Coefficient}

\subsection{2-Dimensional Diffraction}

2-Dimensional diffraction is when the incident ray is perpendicular to the wedge and geometry is invariant along the wedge dimension. The diffraction cone becomes a disk and lies only on the normal plane to the wedge. It's simulated here using 3-D FDTD code by setting $\beta^{\prime}=\beta=\frac{\pi}{2}$.

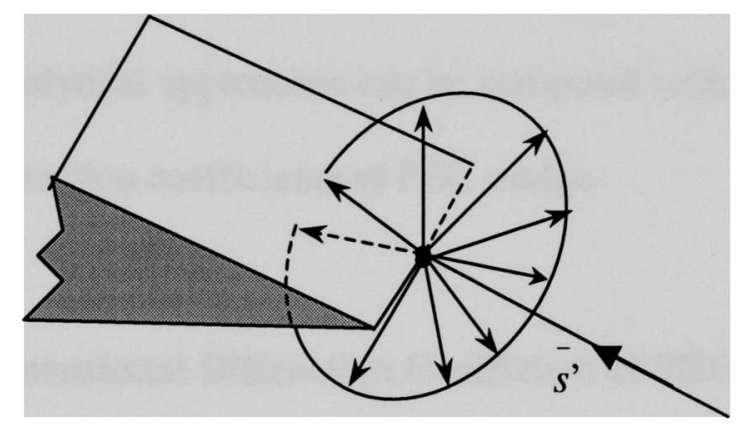

Figure 4.1 2-Dimensional diffraction disk

We can just focus on this plane containing incident ray and all diffraction rays as the $\mathrm{x}-\mathrm{y}$ plane and set the wedge as the $\mathrm{z}$ axis.

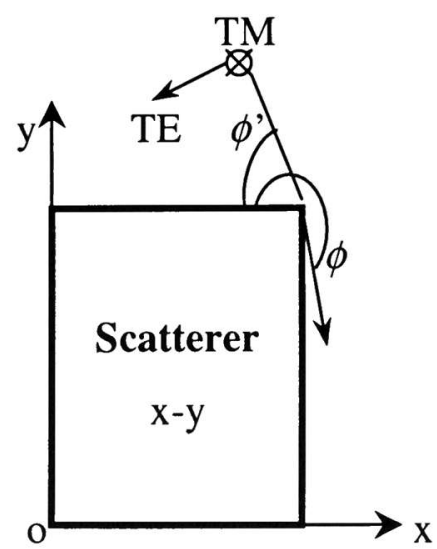

Figure 4.2 TM and TE polarization in 2-dimensional diffraction 
Using the ray-fixed coordinate system in 3-dimensional, $\bar{\beta}$, and $\bar{\beta}$ are in the $\mathrm{z}$ direction and referred to as the TM polarization. $\bar{\phi}$ and $\bar{\phi}$ on the $\mathrm{x}-\mathrm{y}$ plane, normal to the incident and diffracted ray respectively, are referred to as the TE polarization. $D_{s}$ is the soft diffraction coefficient for the $\bar{\beta}$ polarization in 3-dimensional and TM polarization for 2-dimensional diffraction. $D_{h}$ is the hard diffraction coefficient for the $\bar{\phi}$ polarization in 3-dimensional and TE polarization for 2-dimensional diffraction.

\subsection{2-Dimensional Diffraction Coefficient of PEC Wedge}

Numerical and analytical approaches can be compared with each other to validate the FDTD calculated diffraction coefficients of PEC wedge.

\subsubsection{Asymptotic 2-Dimensional Diffraction Coefficient of PEC Wedge}

Because of $\beta=90^{\circ}, \sin \beta=1$, distance parameters $\mathrm{L}=\mathrm{s}, s \cdot \sin ^{2} \beta=s$. The soft and hard diffraction coefficients become to

$$
\begin{array}{r}
D_{s}=\frac{-e^{-j \pi / 4}}{2 n \sqrt{2 \pi k}} \\
\left\{\cot \left[\frac{\pi+\left(\phi-\phi^{\prime}\right)}{2 n}\right] F\left[k s a^{+}\left(\phi-\phi^{\prime}\right)\right]\right. \\
+\cot \left[\frac{\pi-\left(\phi-\phi^{\prime}\right)}{2 n}\right] F\left[k s a^{-}\left(\phi-\phi^{\prime}\right)\right] \\
\\
-\cot \left[\frac{\pi+\left(\phi+\phi^{\prime}\right)}{2 n}\right] F\left[k s a^{+}\left(\phi+\phi^{\prime}\right)\right] \\
\left.-\cot \left[\frac{\pi-\left(\phi+\phi^{\prime}\right)}{2 n}\right] F\left[k s a^{-}\left(\phi+\phi^{\prime}\right)\right]\right\}
\end{array}
$$




$$
\begin{aligned}
D_{h}=\frac{-e^{-j \pi / 4}}{2 n \sqrt{2 \pi k}} & \left\{\cot \left[\frac{\pi+\left(\phi-\phi^{\prime}\right)}{2 n}\right] F\left[k s a^{+}\left(\phi-\phi^{\prime}\right)\right]\right. \\
& +\cot \left[\frac{\pi-\left(\phi-\phi^{\prime}\right)}{2 n}\right] F\left[k s a^{-}\left(\phi-\phi^{\prime}\right)\right] \\
& +\cot \left[\frac{\pi+\left(\phi+\phi^{\prime}\right)}{2 n}\right] F\left[k s a^{+}\left(\phi+\phi^{\prime}\right)\right] \\
& \left.+\cot \left[\frac{\pi-\left(\phi+\phi^{\prime}\right)}{2 n}\right] F\left[k s a^{-}\left(\phi+\phi^{\prime}\right)\right]\right\}
\end{aligned}
$$

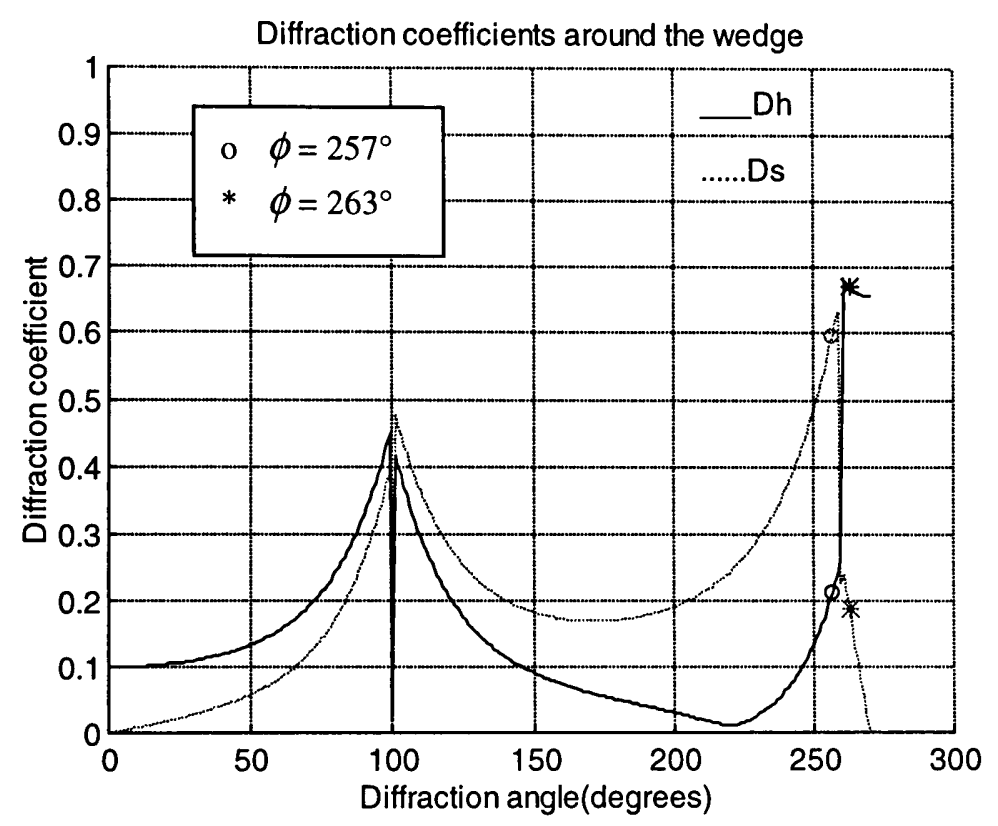

Figure 4.3 Asymptotic soft and hard diffraction coefficients at $850 \mathrm{MHz}$ all around the wedge in $\mathrm{R}=0.8 \mathrm{~m}$ from $\phi^{\prime}=80^{\circ}$ incidence

In the TM polarization case, diffraction coefficient $D_{s}$ approaches to 0 as the angle tends to the surfaces of the wedge. This is because the tangential electric fields must be 0 on the surface of the conducting wedge. The electrical fields normal to the surface for the TE polarization components are not zero. 


\subsubsection{2-Dimensional Diffraction Coefficient of PEC Wedge Using FDTD method}

The diffraction coefficient in shadow region is of practical interest for diffraction phenomenon. The incident plane wave is $80^{\circ}$ from the surface of the wedge. Find the soft and hard diffraction coefficients at $263^{\circ}$ and $257^{\circ}$ around the shadow boundary of $260^{\circ}$ as in Figure 4.3 for 2-dimensional normal incidence.

$$
\begin{gathered}
\phi_{f d t d}^{i}=100^{\circ} \\
\theta_{f d d d}^{i}=90^{\circ}
\end{gathered}
$$

The 3-dimensional FDTD software simulates the 2-D scatterer case inside the problem space as the practical object and helps to get time domain waveforms. To create a preliminary structure all possible coming fields and the time for them to arrive at the receiver need to be considered. To save memory and time, it could be possible to make some scattered fields next to the diffraction pulse reach the receiver at almost the same time which are not concentrated outside time-gating range.

Define cell size $\Delta x=\Delta y=\Delta z=\Delta d=1.41 \mathrm{~cm}$ for $\frac{\Delta d}{\lambda}=\frac{1}{12.5}$ at $1.7 \mathrm{GHz}$, time step $\Delta t=27.188 \mathrm{ps}$. Incident Gaussian plane wave has modulated frequency $f_{0}=850 \mathrm{MHz}$, pulse width $\beta=32$ time steps lasting $1.74 \mathrm{~ns}$, amplitude $\mathrm{A}=1 \mathrm{~V} / \mathrm{m}$. The observation point $\mathrm{R}$ is $0.8 \mathrm{~m}$ distance from the point of diffraction $\mathrm{Q}$. 


\subsubsection{Total Fields and Scattered Fields}

In the whole problem space of FDTD using scattered field formulation, the electrical and magnetic fields are

$$
\begin{aligned}
& E=E^{\text {total }} \equiv E^{\text {incident }}+E^{\text {scattered }} \\
& H=H^{\text {total }} \equiv H^{\text {incident }}+H^{\text {scattered }}
\end{aligned}
$$

The total field is the scattered field added to the incident field. In the scatterer the scattered fields are subject to the Maxwell equations for the specified medium while outside the scatterer they satisfy the free space Maxwell equations. The incident fields always propagate in free space throughout the problem space even passing through the scatterer [2].

In incident shadow region behind the structure there is no incident field, the total fields at the receiver will be the scattered fields. But equations (4.8) and (4.9) make the total fields different from the scattered fields by the incident field. In incident lit region there is incident field, the difference between the total fields and scattered fields is true. So in the incident shadow region the total field is real scattered field. In incident lit region the scattered field is the real scattered field in FDTD calculation.

For the above example, sample total electrical fields at $263^{\circ}$ diffraction angle and sample scattered fields at $257^{\circ}$ for $80^{\circ}$ incidence. 
4.2.2.2 2-Dimensional Diffraction Coefficient of PEC Wedge in Shadow Region III around Incident Shadow Boundary

The 2-D case is simulated using FDTD 3-dimensional code.

$$
\begin{aligned}
& X=142 \\
& Y=116 \\
& Z=220 \\
& X=80 \\
& y=86 \\
& Z=200 \\
& R(102,45,110)
\end{aligned}
$$

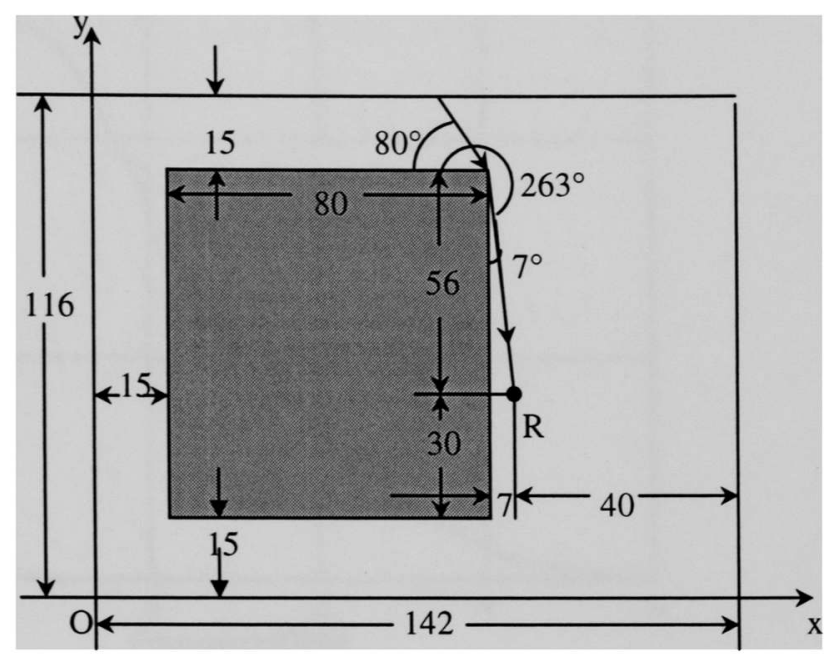

Figure 4.4 Geometry of structure and problem space to get 2-dimensional diffraction coefficient for $\phi^{\prime}=80^{\circ}$ and $\phi=263^{\circ}$ in $\mathrm{R}=0.8 \mathrm{~m}$

1) 2-Dimensional Soft Diffraction Coefficient $D_{s}$ for $\phi^{\prime}=80^{\circ}, \phi=263^{\circ}$

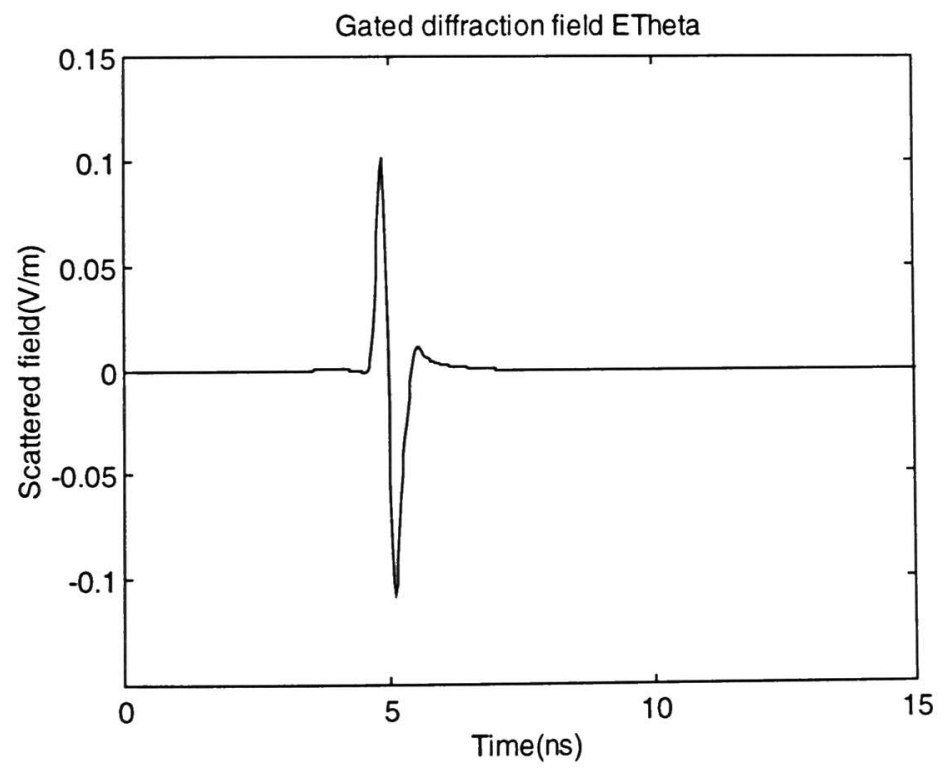

Figure 4.5 Diffraction field in TM polarization for $\phi^{\prime}=80^{\circ}, \phi=263^{\circ}$ in $\mathrm{R}=0.8 \mathrm{~m}$ 


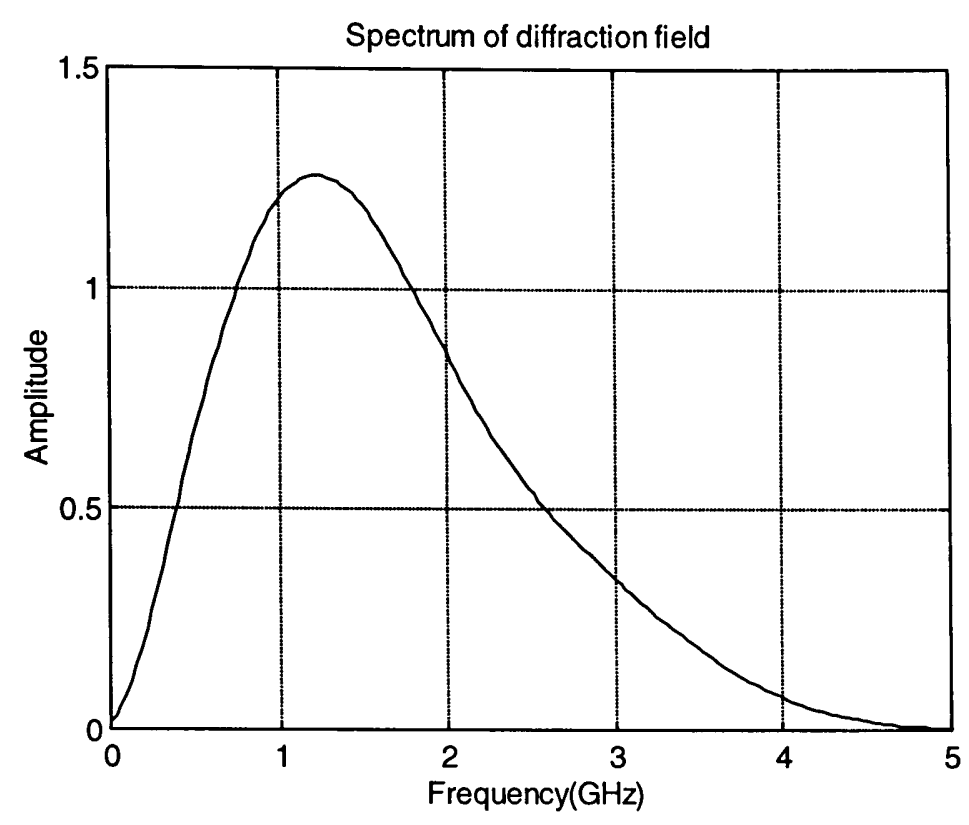

Figure 4.6 Spectrum of the diffraction field in TM polarization for $\phi^{\prime}=80^{\circ}, \phi=263^{\circ}$ in $\mathrm{R}=0.8 \mathrm{~m}$

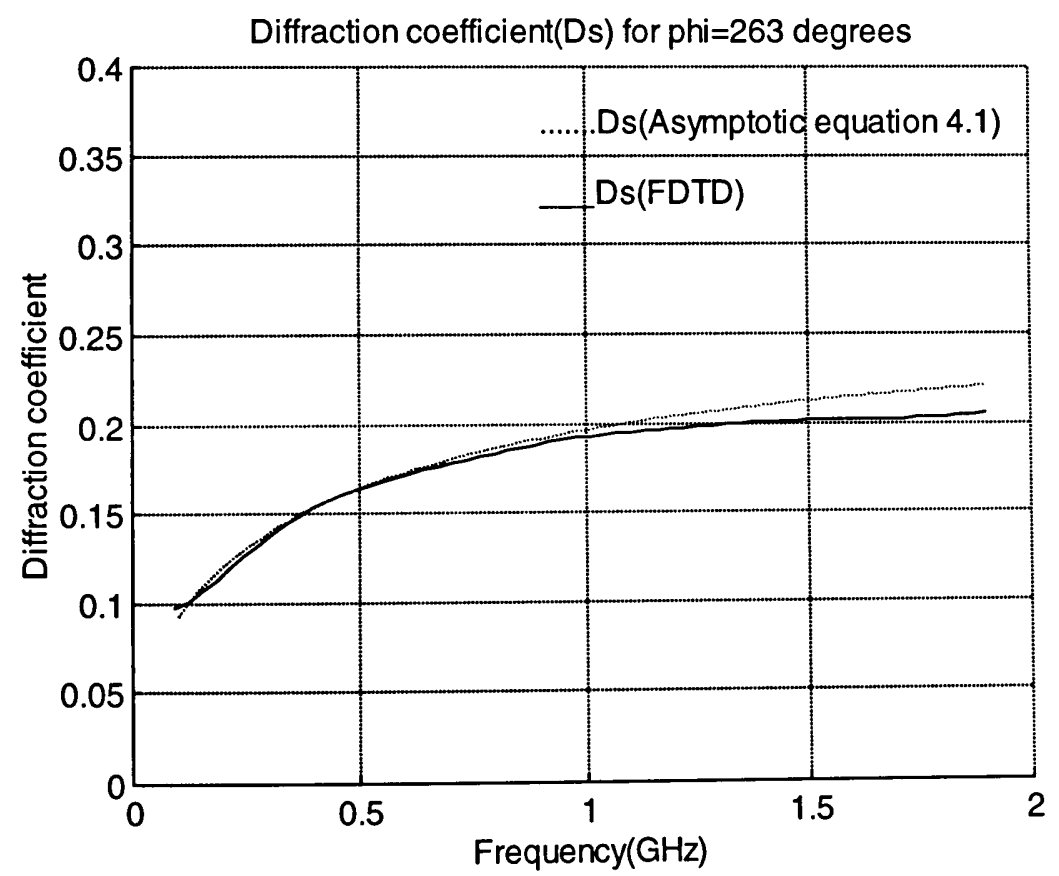

Figure 4.7 2-Dimensional soft diffraction coefficient of PEC wedge for $\phi^{\prime}=80^{\circ}$,

$$
\phi=263^{\circ} \text { in } \mathrm{R}=0.8 \mathrm{~m}
$$


2) 2-Dimensional Hard Diffraction Coefficient $D_{h}$ for $\phi^{\prime}=80^{\circ}, \phi=263^{\circ}$

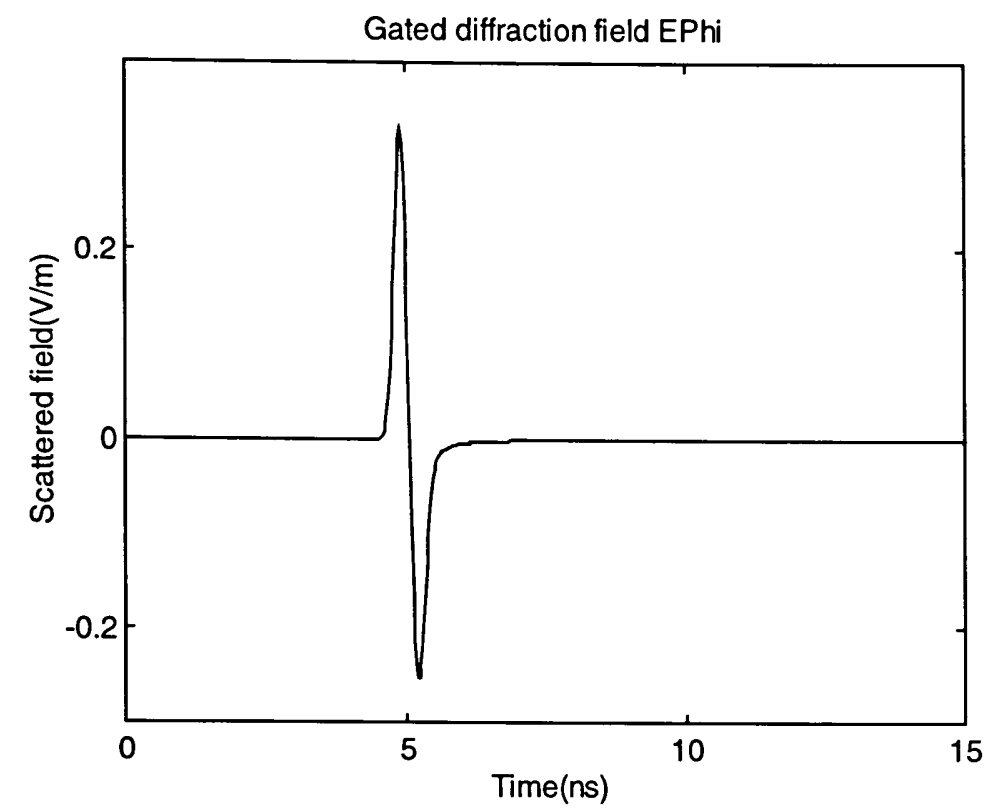

Figure 4.8 Diffraction field in TE polarization for $\phi^{\prime}=80^{\circ}, \phi=263^{\circ}$ in $\mathrm{R}=0.8 \mathrm{~m}$

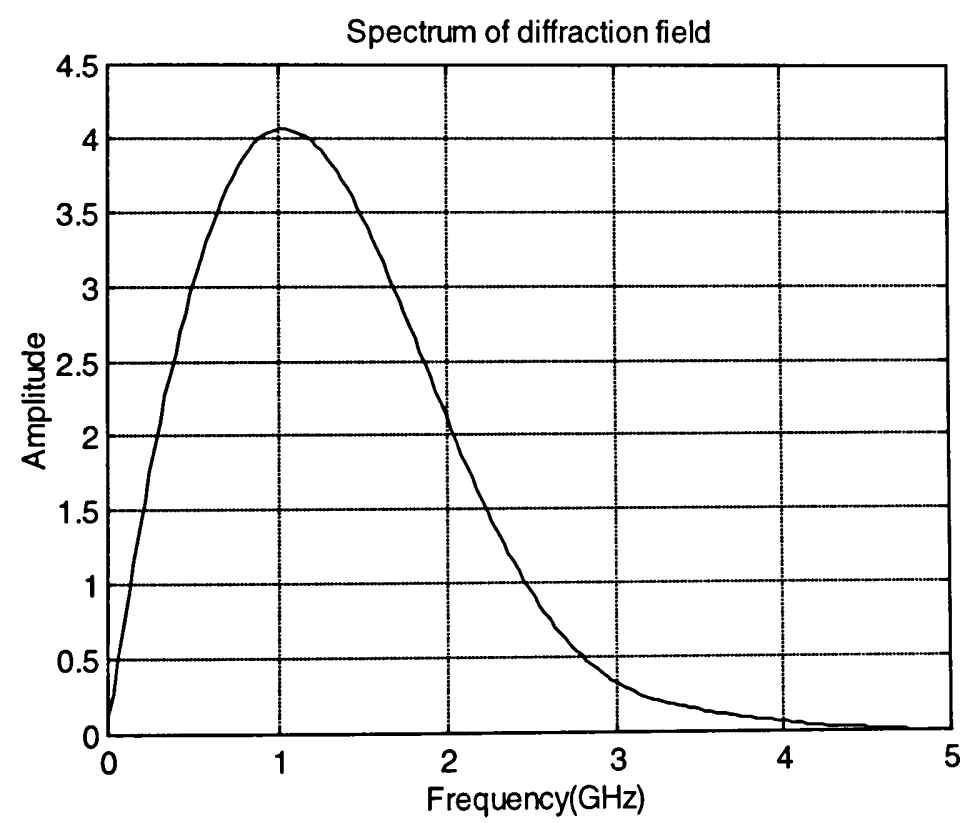

Figure 4.9 Spectrum of the diffraction field in TE polarization for $\phi^{\prime}=80^{\circ}, \phi=263^{\circ}$ in $\mathrm{R}=0.8 \mathrm{~m}$ 


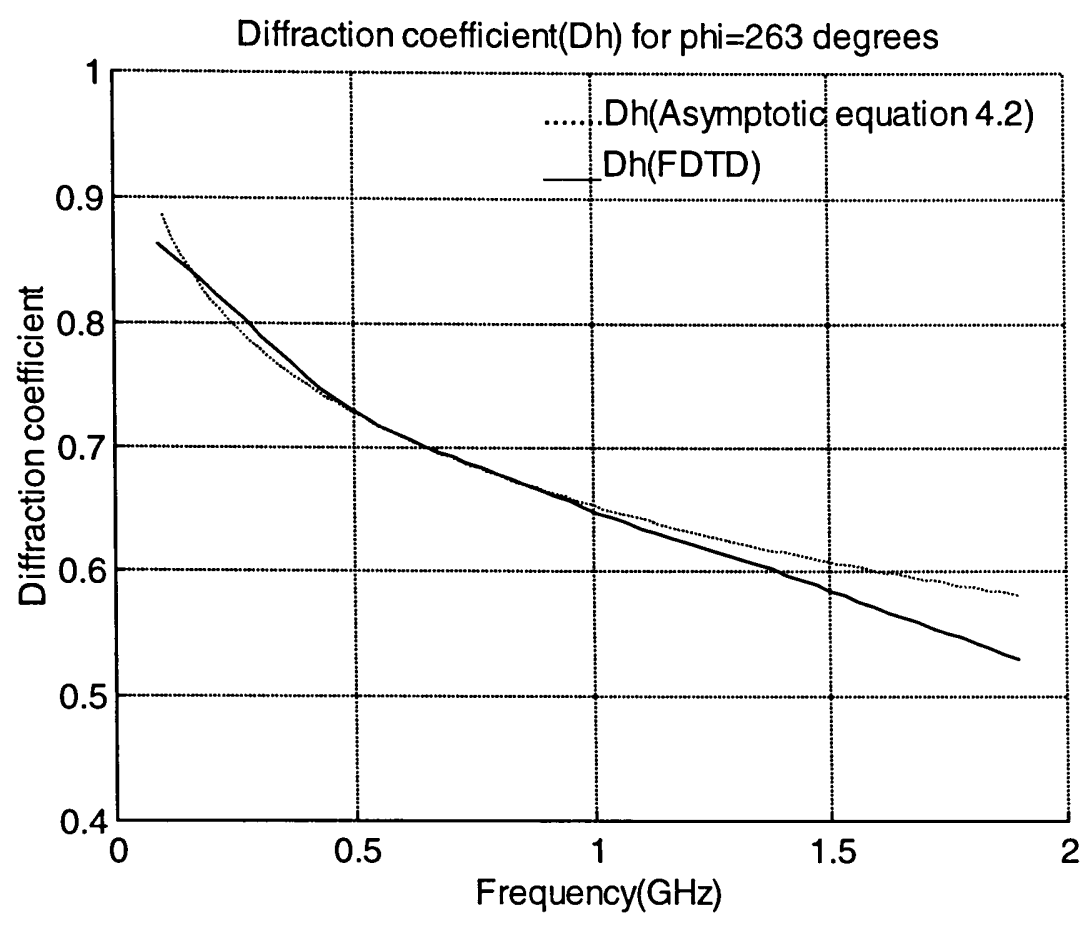

Figure 4.10 2-Dimensional hard diffraction coefficient of PEC wedge for $\phi^{\prime}=80^{\circ}, \phi=263^{\circ}$ in $\mathrm{R}=0.8 \mathrm{~m}$

The quantization error exits in terms of the angle of the observation point and the distance from the wedge. When the diffraction angle is close to the wedge surface or the distance is close to the point of diffraction on the wedge, two cells in the neighbor would have differences about the angle and distance value. When the observation point is around the shadow boundaries little diffraction angle difference would make the diffraction coefficients changed rapidly. Define smaller cell size can improve this error. 
4.2.2.3 2-Dimensional Diffraction Coefficient of PEC Wedge in Region II around Incident Shadow Boundary

$X=148$

$\mathrm{Y}=116$

$\mathrm{Z}=220$

$\mathrm{x}=80$

$\mathrm{y}=86$

$z=200$

$\mathrm{R}(108,46,110)$

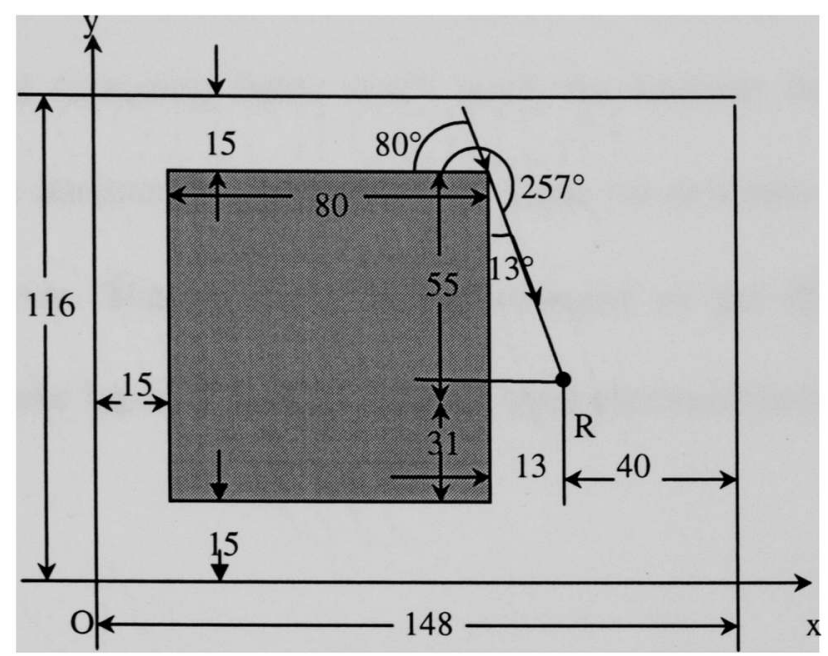

Figure 4.11 Geometry of structure and problem space to get 2-dimensional diffraction coefficient for $\phi^{\prime}=80^{\circ}$ and $\phi=257^{\circ}$ in $\mathrm{R}=0.8 \mathrm{~m}$

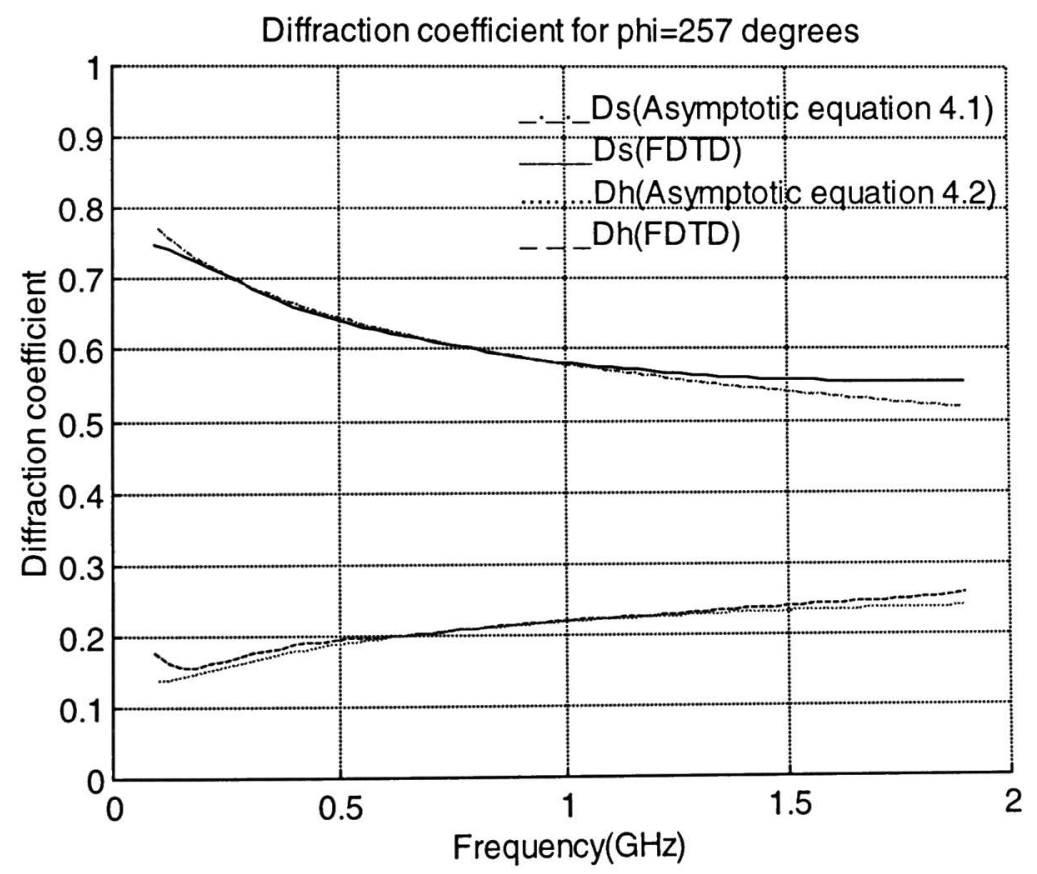

Figure 4.12 2-Dimensional soft and hard diffraction coefficients of PEC wedge for $\phi^{\prime}=80^{\circ}, \phi=257^{\circ}$ in $\mathrm{R}=0.8 \mathrm{~m}$ 


\subsection{2-Dimensional Diffraction Coefficient of Dielectric Wedge}

From the geometry of figure 4.6 to get diffraction coefficients at $\phi=263^{\circ}$ in $0.8 \mathrm{~m}$ from $80^{\circ}$ incidence, the internal scattering fields won't reach the receiver for wedge material $\left(\varepsilon_{r}, \sigma=12,0.1\right)$ and the attenuation constant is very large for structure material $\left(\varepsilon_{r}, \sigma=100,100\right)$ as a conductor. The geometry is not changed to get diffraction coefficients at the receiver for these types of wedges. Sample total electrical fields in this incident shadow region.

1) 2-Dimensional Soft Diffraction Coefficient of Wedge $\left(\varepsilon_{r}, \sigma=12,0.1\right)$ at $\phi^{\prime}=80^{\circ}$, $\phi=263^{\circ}$

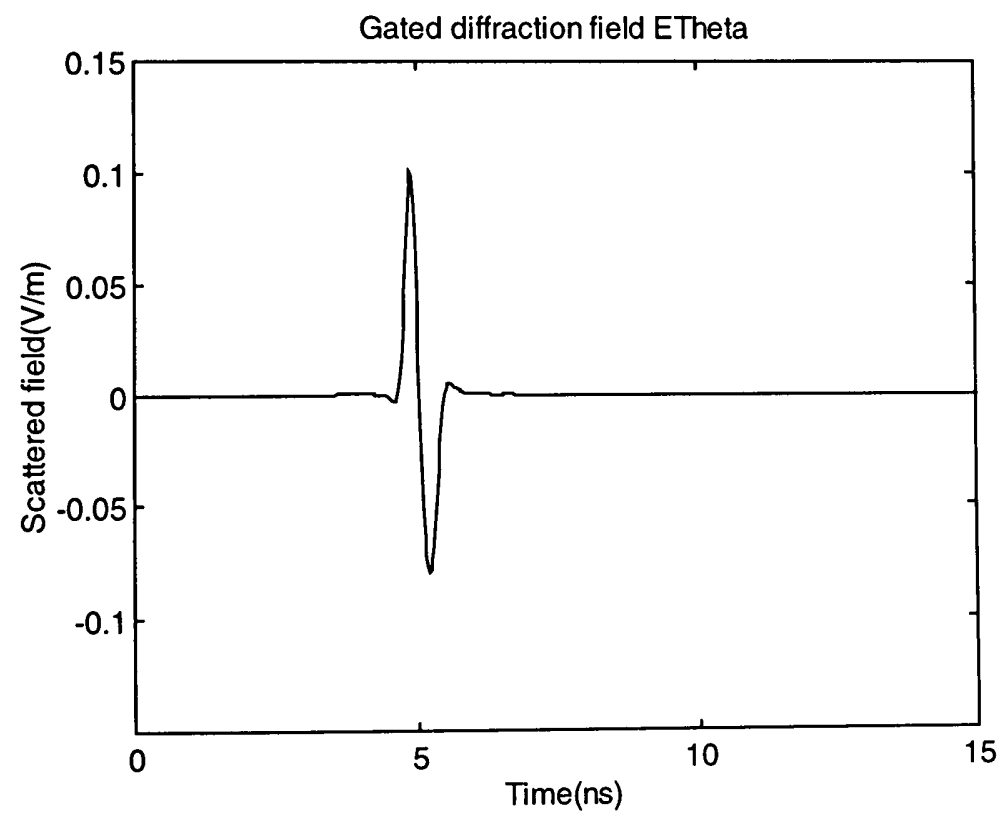

Figure 4.13 Diffraction field in TM polarization for $\phi^{\prime}=80^{\circ}, \phi=263^{\circ}$ in $\mathrm{R}=0.8 \mathrm{~m}$ for wedge $\left(\varepsilon_{r}, \sigma=12,0.1\right)$ 


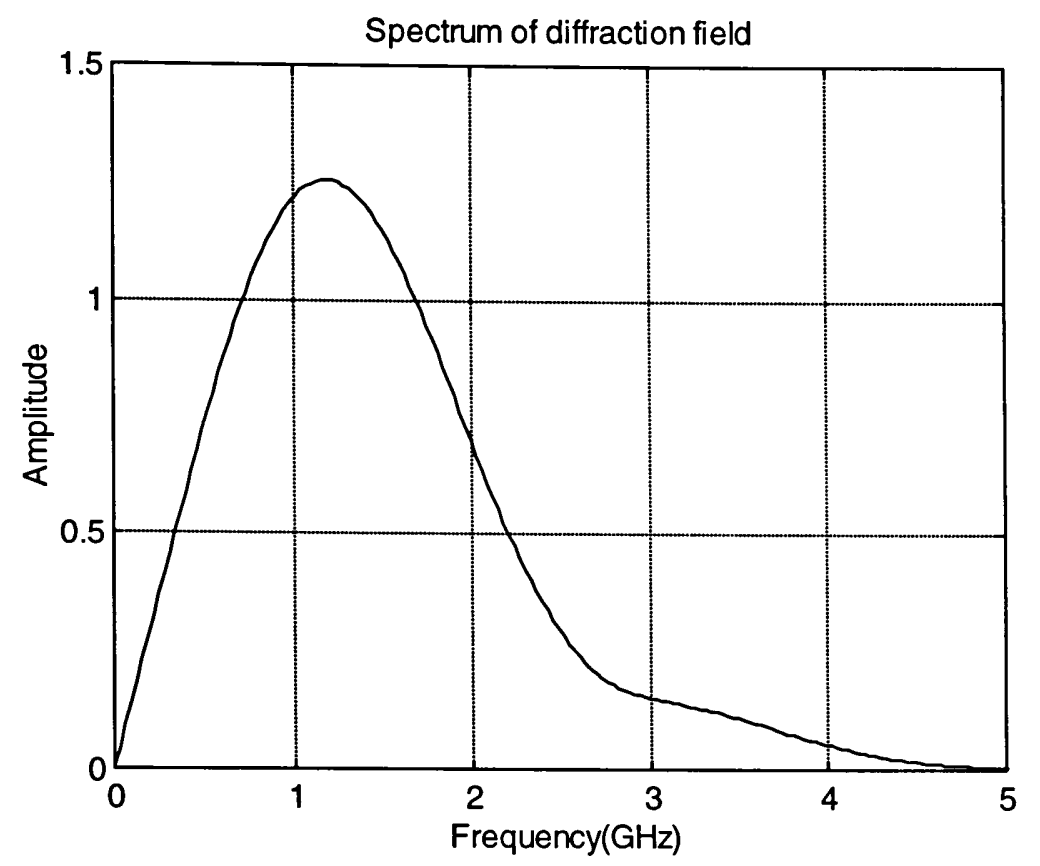

Figure 4.14 Spectrum of the diffraction field from TM polarization for $\phi^{\prime}=80^{\circ}, \phi=263^{\circ}$ in $\mathrm{R}=0.8 \mathrm{~m}$ for wedge $\left(\varepsilon_{r}, \sigma=12,0.1\right)$

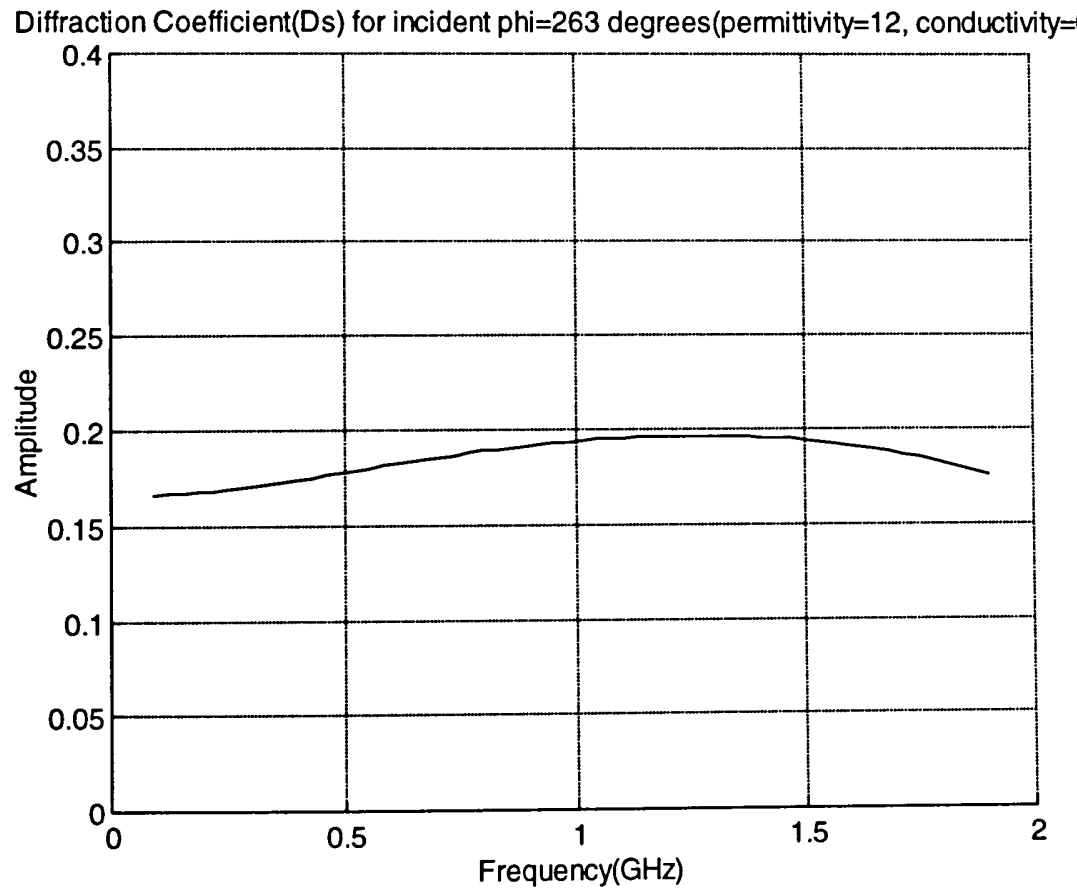

Figure 4.15 2-Dimensional soft diffraction coefficient for $\phi^{\prime}=80^{\circ}, \phi=263^{\circ}$ in $\mathrm{R}=0.8 \mathrm{~m}$ for wedge $\left(\varepsilon_{r}, \sigma=12,0.1\right)$ 
2) 2-Dimensional Hard Diffraction Coefficient of Wedge $\left(\varepsilon_{r}, \sigma=12,0.1\right)$ at $\phi^{\prime}=80^{\circ}$, $\phi=263^{\circ}$

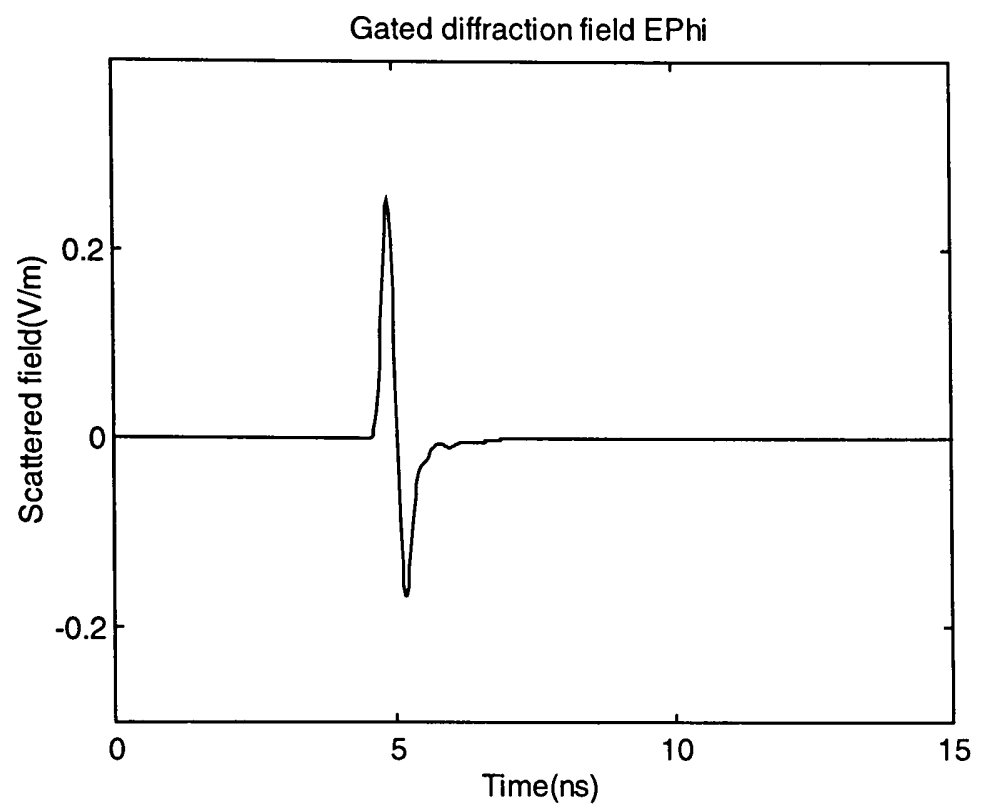

Figure 4.16 Diffraction field in TE polarization for $\phi^{\prime}=80^{\circ}, \phi=263^{\circ}$ in $\mathrm{R}=0.8 \mathrm{~m}$ for wedge $\left(\varepsilon_{r}, \sigma=12,0.1\right)$

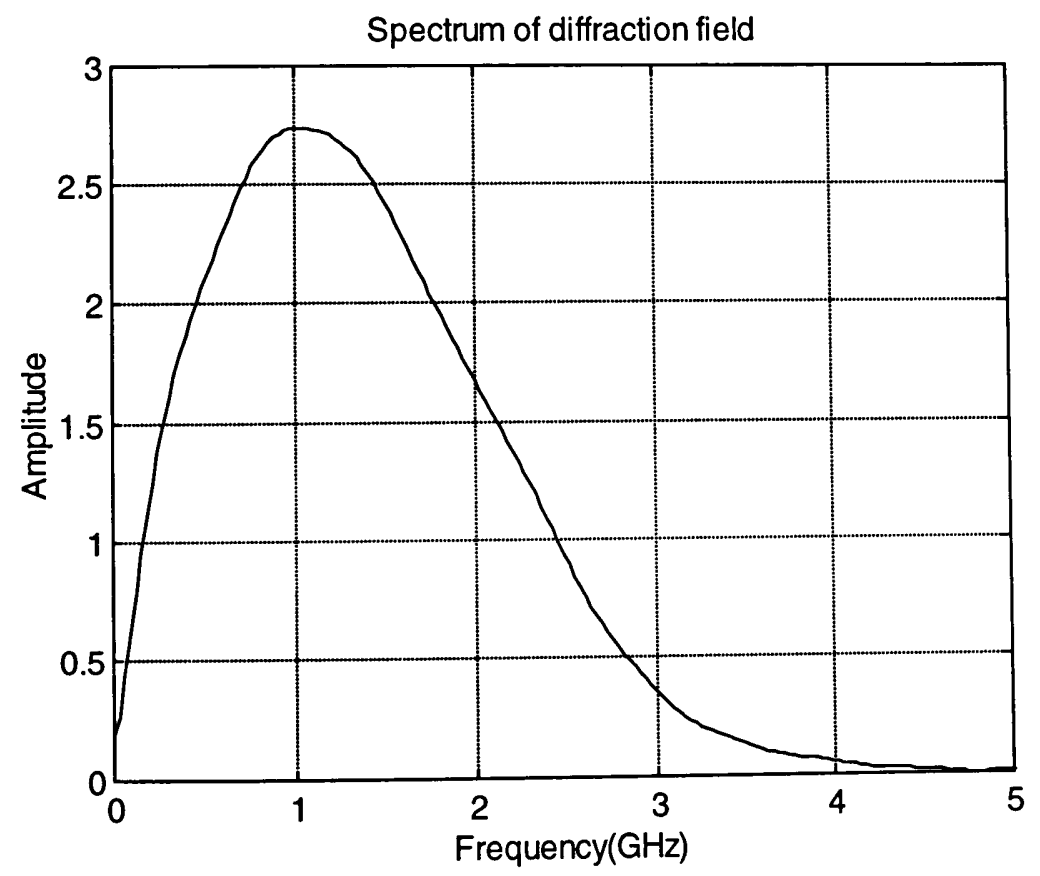

Figure 4.17 Spectrum of the diffraction field from TE polarization for $\phi^{\prime}=80^{\circ}, \phi=263^{\circ}$ in $\mathrm{R}=0.8 \mathrm{~m}$ for wedge $\left(\varepsilon_{r}, \sigma=12,0.1\right)$ 


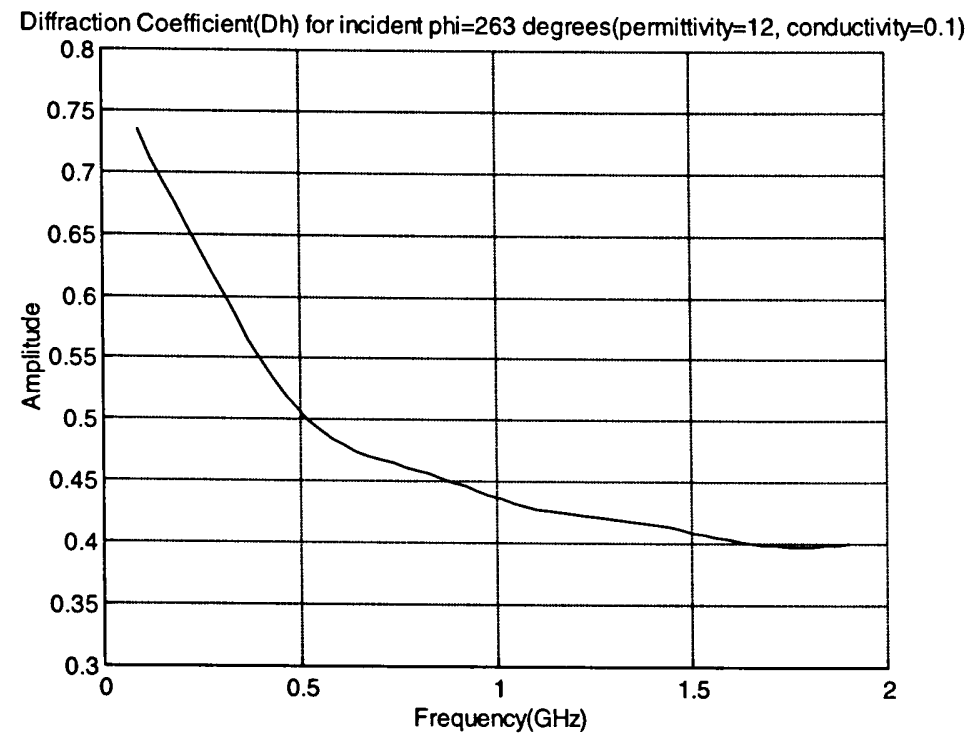

Figure 4.18 2-Dimensional hard diffraction coefficient for $\phi^{\prime}=80^{\circ}, \phi=263^{\circ}$ in $\mathrm{R}=0.8 \mathrm{~m}$ for wedge $\left(\varepsilon_{r}, \sigma=12,0.1\right)$

Compare the soft and hard diffraction coefficients with PEC, conductor and dielectric wedges.

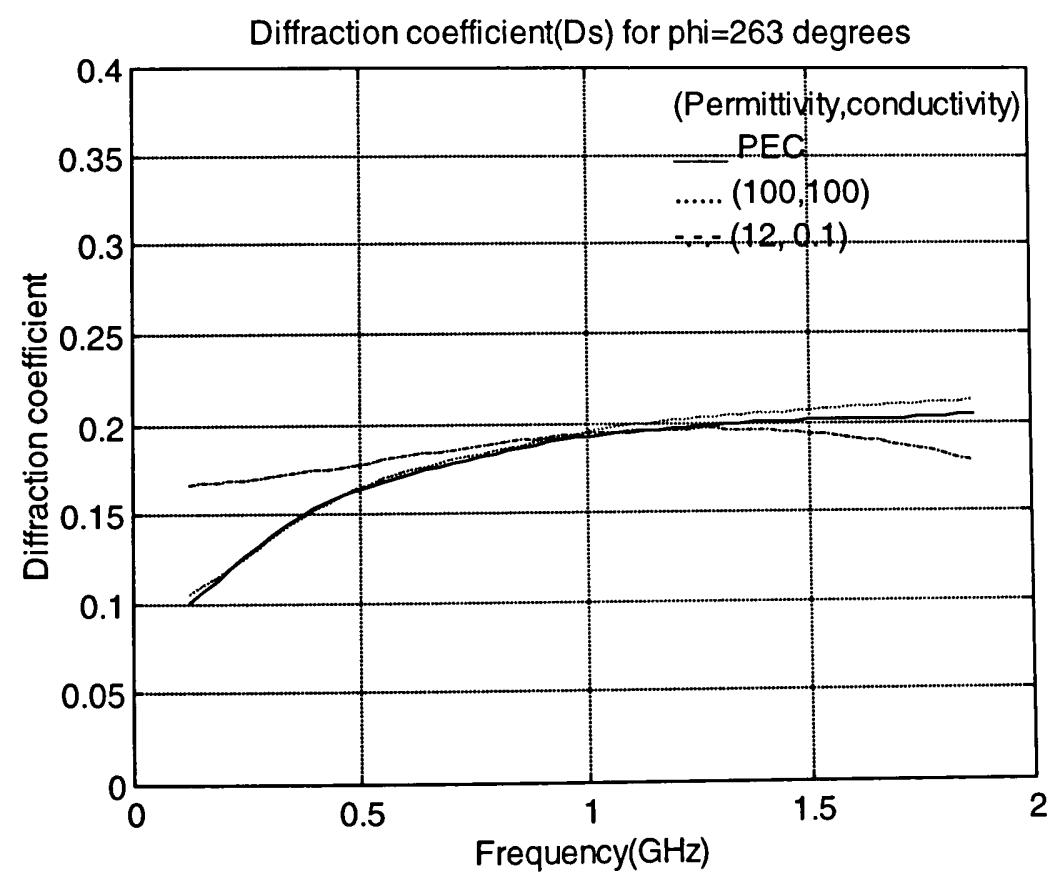

Figure 4.19 2-Dimensional soft diffraction coefficient for $\phi^{\prime}=80^{\circ}, \phi=263^{\circ}$ in $\mathrm{R}=0.8 \mathrm{~m}$ for wedges of PEC, $\left(\varepsilon_{r}, \sigma=100,100\right)$ and $\left(\varepsilon_{r}, \sigma=12,0.1\right)$ 


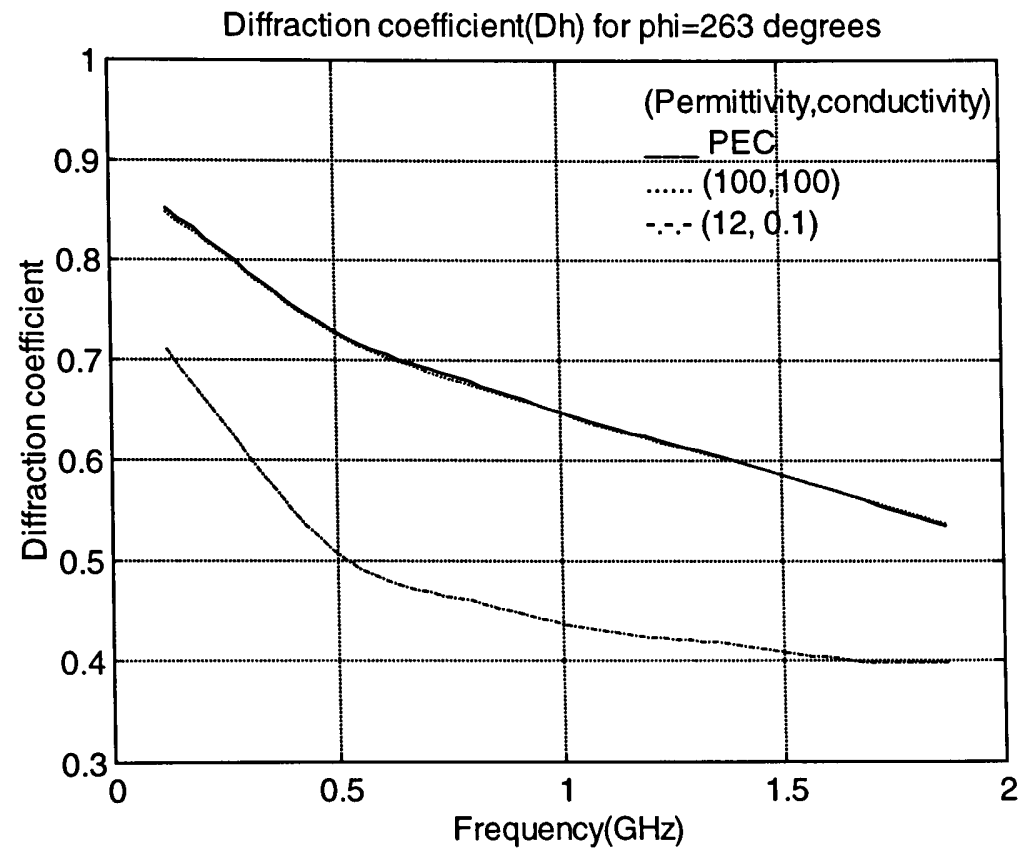

Figure 4.20 2-Dimensional hard diffraction coefficient for $\phi^{\prime}=80^{\circ}, \phi=263^{\circ}$ in $\mathrm{R}=0.8 \mathrm{~m}$ for wedges of PEC, $\left(\varepsilon_{r}, \sigma=100,100\right)$ and $\left(\varepsilon_{r}, \sigma=12,0.1\right)$ 


\section{Chapter 5}

\section{Conclusions and Future Work}

\subsection{Conclusions}

Numerical calculations of diffraction coefficients using FDTD method are presented for PEC, conductor and dielectric right angle wedges.

Geometries of PEC structure and problem space are shown for 3-dimensional and 2-dimensional diffraction. The purpose of the geometry is to get a far field equivalent diffraction pulse from the wedge separated from all other scattered fields. When using Fourier transform the results of soft and hard diffraction coefficients in wide frequency band are close to the analytical solutions from Uniform Theory of Diffraction illustrated by above cases.

Results presented here are primarily limited to diffraction in the lit region. Based on the agreements of using FDTD method to the asymptotic expressions for PEC wedges, applying the same structures and extending the wedge properties $\left(\varepsilon_{r}, \sigma\right)$ to some types of conductor and dielectric materials for diffraction coefficients of building wedges will have quite feasibility. Data for different wedge types are collected particularly at frequencies of $850 \mathrm{MHz}$ and $1.7 \mathrm{GHz}$ used in wireless communication applications. 


\subsection{Future Work}

Experimental measurement can obtain accurate time domain diffraction fields to validate diffraction coefficients of PEC wedge with asymptotic using good conductors. The dielectric diffraction coefficients using FDTD method without analytical comparison could be validated by measurements. 


\section{References}

[1] K. S. Yee, "Numerical Solution of initial boundary value problems involving Maxwell's equations in isotropic media", IEEE Transactions on Antennas and Propagation, vol. Ap-14, pp. 302-307, May 1966.

[2] Karl S. Kunz, Raymond J. Luebbers, The Finite Difference Time Domain Method for Electromagnetics, CRC Press, 1993.

[3] User's Manual for XFDTD, Remcom Inc., Version 5.0, December 1998.

[4] Korada Umashankar, Allen Taflove, "A novel Method to Analyze Electromagnetic Scattering of Complex Objects", IEEE Transactions on Electromagnetic Compatibility, vol. EMC-24, No. 4, pp. 397-405, November 1982.

[5] Allen Taflove, "Review of FD-TD Numerical Modeling of Electromagnetic Wave Scattering and Radar Cross Section", Proceeding of the IEEE, vol. 77, No. 5, pp. 682698, May 1989.

[6] Raymond J. Luebbers, Karl S. Kunz, Michael Schneider, Forrest Hunsberger, "A finite-Difference Time Domain Near Zone to Far Zone Transformation", IEEE Transactions on Antennas and Propagation", vol. 39, No. 4, pp. 429-433, April 1991.

[7] D. A. Mcnamara, C.W. I. Pistorius, J.A.G. Malherbe, Introduction to the Uniform Geometrical Theory of Diffraction, Artech house, 1990.

[8] Robert G. Kouyoumjian, Prabhakar H. Pathak, "A Uniform Geometrical Theory of Diffraction for an Edge in a Perfectly Conducting Surface", Proceeding of the IEEE, vol. 62, pp. 1448-1461, November 1974.

[9] V. Anantha, A. Taflove, "Calculation of Diffraction Coefficients of ThreeDimensional Infinite Conducting Wedges Using FDTD”, IEEE Transactions on Antennas and Propagation, vol. 46, No. 11, pp. 1755-1756, November 1998.

[10] G. Stratis, V. Anantha, A. Taflove, "Numerical Calculation of Diffraction Coefficients of Generic Conducting and Dielectric Wedges Using FDTD", IEEE Transactions on Antennas and Propagation, vol. 45, No. 10, pp. 1525-1529, October 1997. 
[11] James C. Rautio, "Experimental Validation of Electromagnetic Software", International Journal of Microwave and Millimeter-Wave Computer-Aided Engineering, vol.1, No., pp. 379-385, 1991.

[12] Kazimierz Siwiak, Radiowave Propagation and Antennas for Personal Communications, Second Edition, Artech House, 1998.

[13] John D. Kraus, Electromagnetics, Fourth Edition, McGraw- Hill, 1992.

[14] Theodore S. Rappaport, Wireless Communications, Prentice Hall, 1996. 